\title{
Study of 3-D Dynamic Roughness Effects on Flow Over a NACA 0012 Airfoil Using Large Eddy Simulations at Low Reynolds Numbers
}

Venkata Subba Sai Satish Guda

Follow this and additional works at: https://researchrepository.wvu.edu/etd

\section{Recommended Citation}

Guda, Venkata Subba Sai Satish, "Study of 3-D Dynamic Roughness Effects on Flow Over a NACA 0012 Airfoil Using Large Eddy Simulations at Low Reynolds Numbers" (2015). Graduate Theses, Dissertations, and Problem Reports. 5719.

https://researchrepository.wvu.edu/etd/5719

This Thesis is protected by copyright and/or related rights. It has been brought to you by the The Research Repository @ WVU with permission from the rights-holder(s). You are free to use this Thesis in any way that is permitted by the copyright and related rights legislation that applies to your use. For other uses you must obtain permission from the rights-holder(s) directly, unless additional rights are indicated by a Creative Commons license in the record and/ or on the work itself. This Thesis has been accepted for inclusion in WVU Graduate Theses, Dissertations, and Problem Reports collection by an authorized administrator of The Research Repository @ WVU. For more information, please contact researchrepository@mail.wvu.edu. 


\title{
Study of 3-D Dynamic Roughness Effects on Flow Over a NACA 0012 Airfoil Using Large Eddy Simulations at Low Reynolds Numbers \\ Venkata Subba Sai Satish Guda
}

\author{
Thesis Submitted to the Benjamin M. Statler College of Engineering and Mineral resources \\ at West Virginia University \\ in partial fulfillment of the requirements for the degree of \\ Master of Science \\ in \\ Mechanical and Aerospace Engineering
}

Approved by

Dr. Wade W. Huebsch, Chair

Dr. John M. Kuhlman

Dr. Gregory J. Thompson

Department of Mechanical and Aerospace Engineering

Morgantown, West Virginia

2015 


\title{
ABSTRACT \\ Study of 3-D Dynamic Roughness Effects on Flow Over a NACA 0012 Airfoil Using Large Eddy Simulations at Low Reynolds Numbers
}

\author{
Venkata Subba Sai Satish Guda
}

There have been several advancements in the aerospace industry in areas of design such as aerodynamics, designs, controls and propulsion; all aimed at one common goal i.e. increasing efficiency -range and scope of operation with lesser fuel consumption. Several methods of flow control have been tried. Some were successful, some failed and many were termed as impractical. The low Reynolds number regime of $10^{4}-10^{5}$ is a very interesting range. Flow physics in this range are quite different than those of higher Reynolds number range. Mid and high altitude UAV's, MAV's, sailplanes, jet engine fan blades, inboard helicopter rotor blades and wind turbine rotors are some of the aerodynamic applications that fall in this range. The current study deals with using dynamic roughness as a means of flow control over a NACA 0012 airfoil at low Reynolds numbers. Dynamic 3-D surface roughness elements on an airfoil placed near the leading edge aim at increasing the efficiency by suppressing the effects of leading edge separation like leading edge stall by delaying or totally eliminating flow separation. A numerical study of the above method has been carried out by means of a Large Eddy Simulation, a mathematical model for turbulence in Computational Fluid Dynamics, owing to the highly unsteady nature of the flow. A user defined function has been developed for the 3-D dynamic roughness element motion. Results from simulations have been compared to those from experimental PIV data. Large eddy simulations have relatively well captured the leading edge stall. For the clean cases, i.e. with the DR not actuated, the LES was able to reproduce experimental results in a reasonable fashion. However DR simulation results show that it fails to reattach the flow and suppress flow separation compared to experiments. Several novel techniques of grid design and hump creation are introduced through this study. 
"The desire to fly is an idea handed down to us by our ancestors who, in their grueling travels across trackless lands in prehistoric times, looked enviously on the birds soaring freely through space, at full speed, above all obstacles, on the infinite highway of the air."

\section{Orville Wright}

"It is possible to fly without motors, but not without knowledge and skill."-

Wilbur Wright 


\section{Acknowledgements}

First and foremost, I would like to express my sincere gratitude to Dr. Huebsch for giving me the wonderful opportunity to work on this interesting and challenging topic. Without his guidance and support as my research advisor, this wouldn't have been possible.

I would like to thank Dr. John Kuhlman and Dr. Gregory Thompson for their wonderful guidance and suggestions. I would like to also thank all the Professors at WVU for enriching my knowledge and giving me a platform to stand on

I would like to thank Dr. Ashish Robert for his day to day guidance and wonderful suggestions.

I would like to sincerely thank my friend and colleague Dr. Chris Griffin for his constant support, guidance and suggestions in my research work. Along with him, I would also like to thank Adam Johnson and Vinay Jakkali for creating a wonderful working environment in the office and for all the fruitful discussions and suggestions.

I would like thank Mr. Don McLaughlin who was very instrumental in helping me setup Fluent on the Mountaineer HPC cluster, without which this work would have been impossible. There were lot of problems and he patiently helped me overcome all the challenges.

Also my sincere thanks to all the support staff of WVU for their help during this work.

I sincerely thank my parents, family and friends for supporting me and helping me to achieve my dreams of obtaining wonderful education at an amazing university. I would like to thank my grandfather Dr. K. Sivananda Murthy for all the wonderful guidance, love and support. Thanks a lot for motivating me and supporting me in challenging situations. 


\section{Contents}

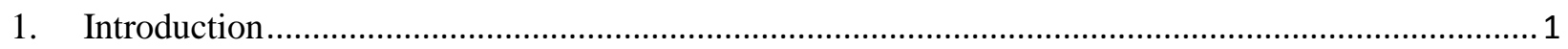

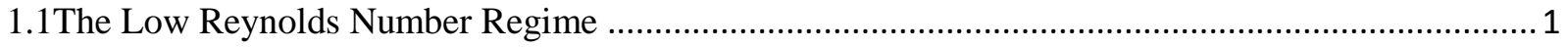

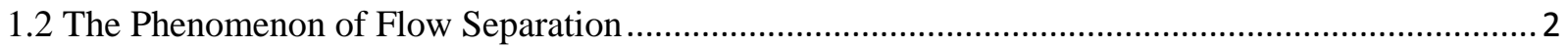

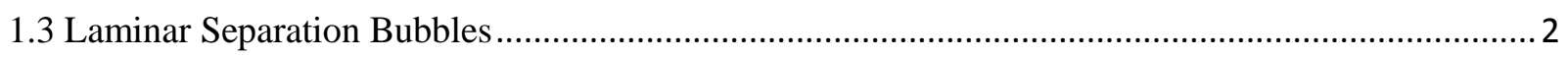

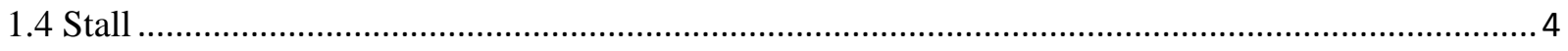

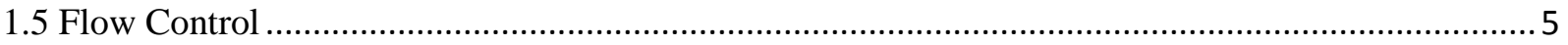

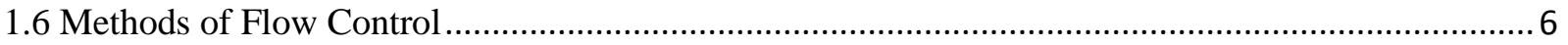

1.7 Flow Control Using Dynamic Roughness (DR) .................................................................. 7

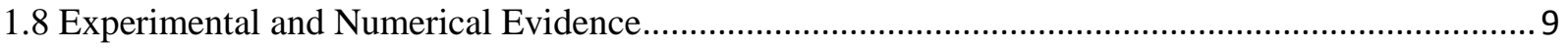

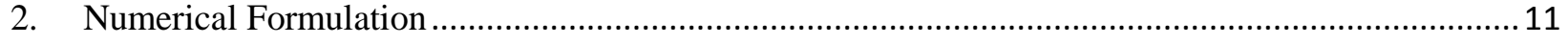

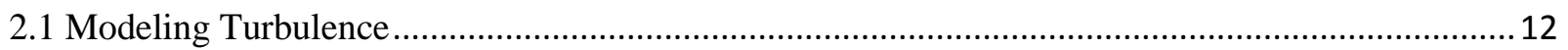

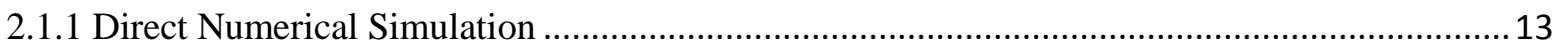

2.1.2 Reynolds Averaged Navier Stokes (RANS) Models .........................................................13

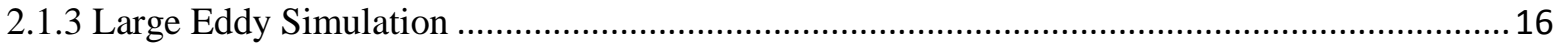

2.2 Need to go for a Large Eddy Simulation .............................................................................. 16

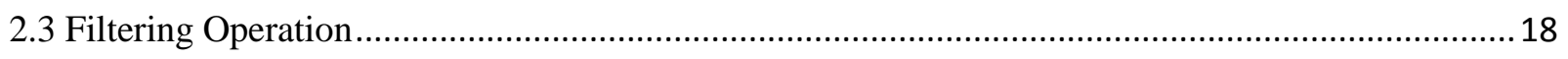

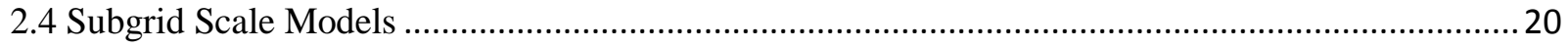

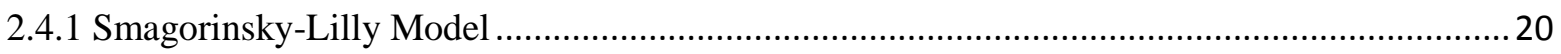

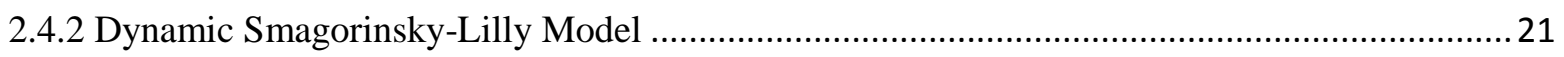

2.4.3 Wall-Adapting Local Eddy-Viscosity (WALE) Model …......................................................23

2.4.4 Dynamic Kinetic Energy Subgrid-Scale Model.................................................................2 24

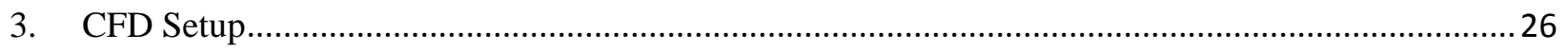

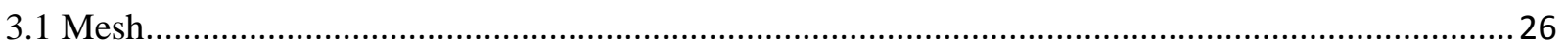

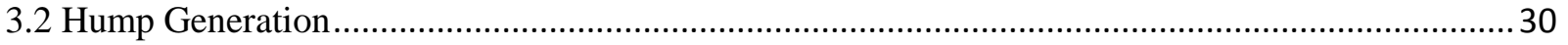

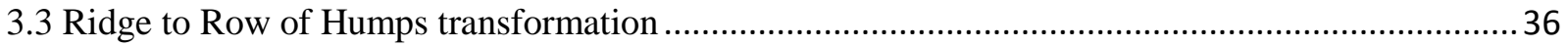

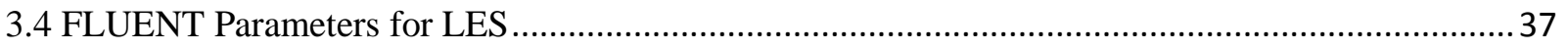

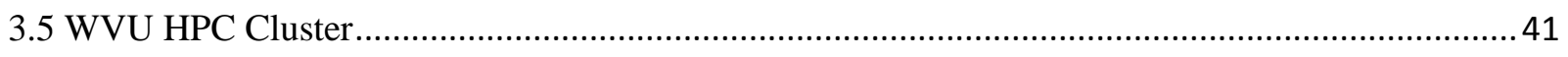

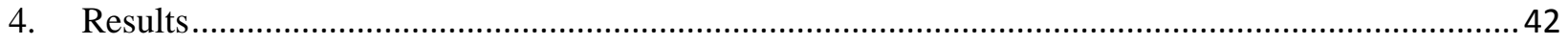

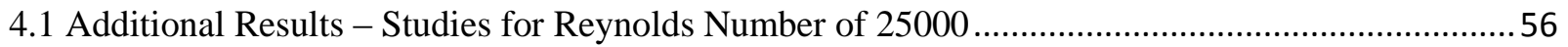


4.2 Additional Results - Study on Order of Transient Formulation

4.3 Additional Results - Study with higher amplitude …..............................................................64

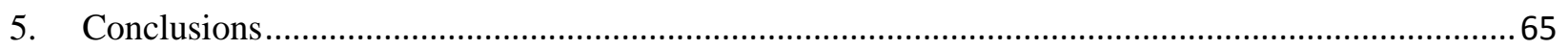

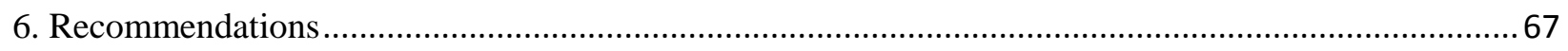

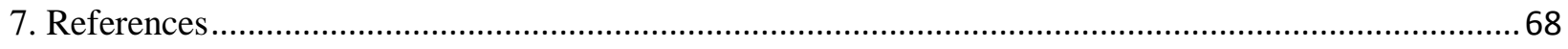

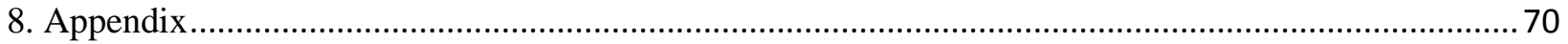




\section{List of Figures}

Figure 1.1 Sketch of a laminar separation bubble (2) ......................................................................

Figure 2.1 RANS and LES comparison of velocity contours, Kim (12) ..............................................17

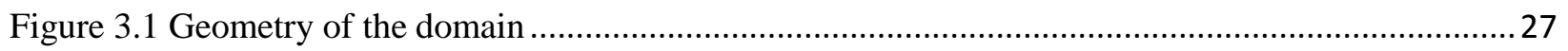

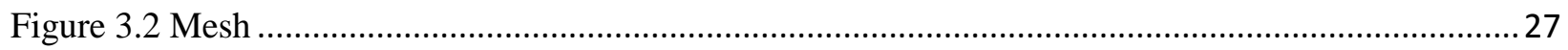

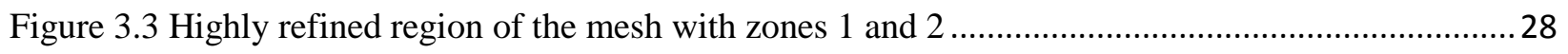

Figure 3.4 Highly refined region of the mesh with zones 1 and 2 (locally enlarged) .............................28

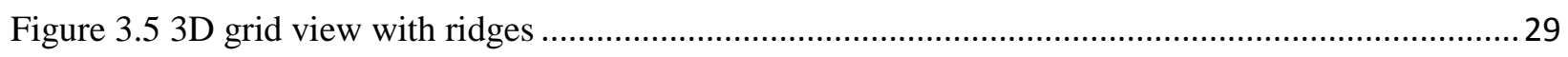

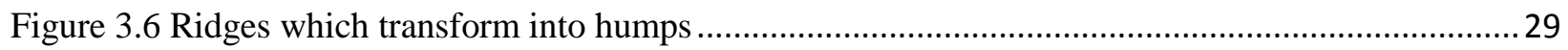

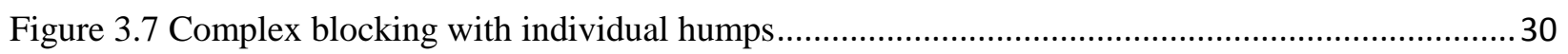

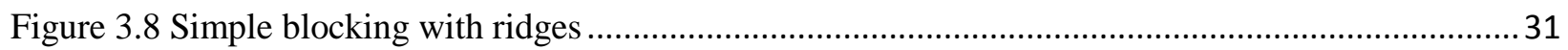

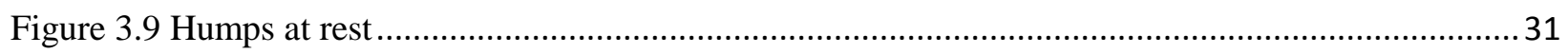

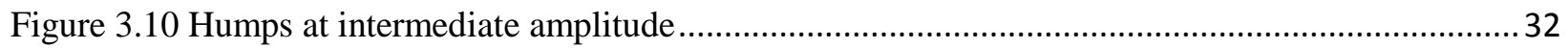

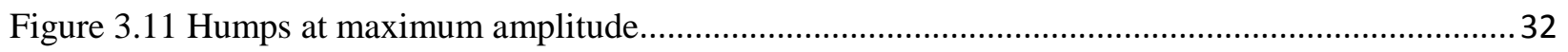

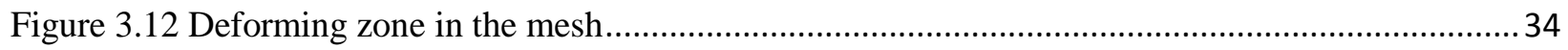

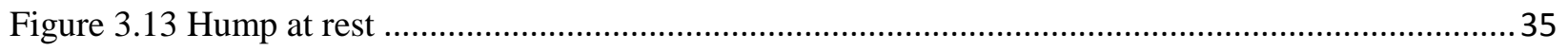

Figure 3.14 Hump in motion................................................................................................... 36

Figure 4.1 Comparison of TKE contours from experiments and simulations (preliminary) for clean case43

Figure 4.2 Comparison of velocity magnitude contours for clean case ..................................................44

Figure 4.3 Comparison of TKE contours for clean case .................................................................. 45

Figure 4.4 Comparison of vorticity contours for clean case …........................................................ 47

Figure 4.5 Velocity magnitude contour plot with vectors (clean) ........................................................ 48

Figure 4.6 Comparison of velocity magnitude contours for DR case ...................................................49

Figure 4.7 Comparison of TKE contours for DR case ....................................................................50

Figure 4.8 Comparison of vorticity contours for DR case ............................................................... 52

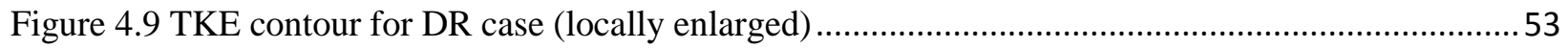

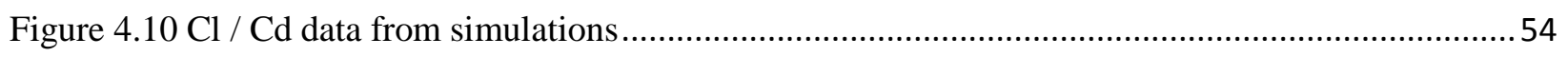

Figure 4.11 Mean wall shear stress from simulations.....................................................................5 55

Figure 4.12 Mean Cp data over DR region from simulations ..............................................................56

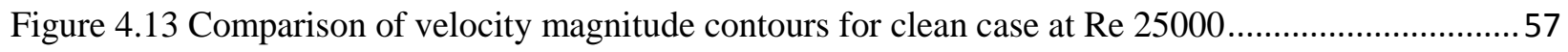

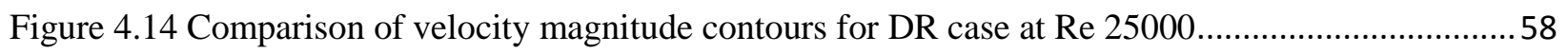

Figure 4.15 Comparison of velocity magnitude contours for clean cases at $\operatorname{Re} 49000$..........................60

Figure 4.16 Comparison of TKE contours for clean cases at Re 49000 ................................................61

Figure 4.17 Comparison of vorticity contours for clean cases at Re 49000 .........................................62

Figure 4.18 Comparison of mean $\mathrm{x}$-wall shear stress for clean cases at $\operatorname{Re} 49000$................................63

Figure 4.19 Comparison of velocity magnitude contours for Re 25000 for DR amplitude of $0.5 \mathrm{~mm}$.......64 


\section{List of Tables}

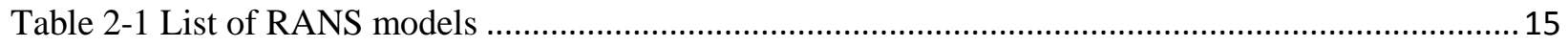

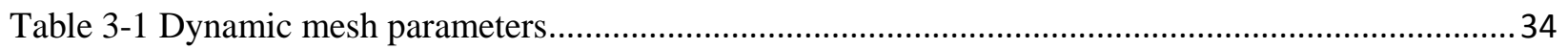

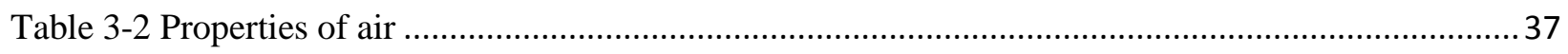

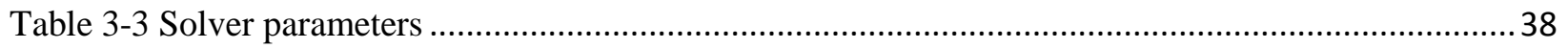

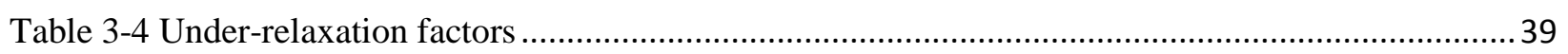

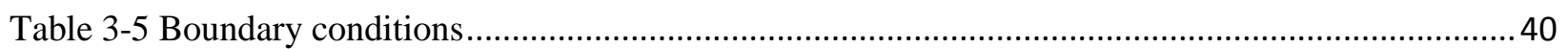

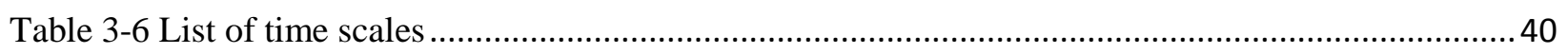

Table 4-1 Height of separated layer (from simulation) ...................................................................... 55 


\section{Introduction}

There have been several advancements in the aviation industry over the last two decades. Advancements in design, aerodynamics, propulsion and controls have totally changed the scenario of this industry in terms of range and scope of operation. Day by day, the innovations in this field have taken man farther and farther in less time. One topic which is currently being greatly focused upon by many scientists and engineers is flow control as a means of increasing aircraft efficiency.

Flow control is basically the ability to manipulate a flow field, over a body, actively or passively to affect a desired change in flow behavior. The potential benefits from this concept range from saving billions of dollars in annual fuel costs for land, air and sea vehicles to achieving economically and environmentally more competitive industrial processes involving fluid flows. From the aerodynamics point of view, extensive research has been going on to get an ideal workable flow control mechanism. The spotlight for the current work is on flow control over an airfoil in the low Reynolds number regime of $10^{4}-10^{5}$. The focus is on flow separation and its control in this regime.

\subsection{The Low Reynolds Number Regime}

The aerodynamic low Reynolds number regime of $10^{4}-10^{5}$ is a very interesting range of study for researchers studying fluid flows. Flow physics in this range are quite different than those of higher Reynolds numbers. According to Carmichael (1), this is the Reynolds number regime in which we find humans and nature together in flight: large soaring birds, remotely piloted aircraft (used for military and scientific sampling, monitoring and surveillance), mid and high altitude UAV's, micro air vehicles (MAV), sailplanes, jet engine fan blades, inboard helicopter rotor 
blades and wind turbine rotors are some of the aerodynamic applications that fall in this range. At low Reynolds numbers, the flow fields can become highly unsteady because of complex flow characteristics due to separation, transition and reattachment. These phenomena dramatically affect the performance of a lifting surface, as well as the analysis of these flow fields.

\subsection{The Phenomenon of Flow Separation}

Wall friction slows down fluid particles in all boundary layers. If the flow is also retarded due to the presence of an adverse pressure gradient, the momentum of the fluid particles will be reduced by both the wall shear and the pressure gradient. In terms of energy principles, the kinetic energy gained at the expense of potential energy in the favorable pressure gradient region is depleted by viscous effects within the boundary layer. In the adverse pressure gradient region, the available kinetic energy is converted to potential energy, but is too small to surmount the pressure gradient and thus the motion of the near wall fluid particles is arrested. At some point, the viscous layer departs or breaks away from the bonding surface. This point is commonly defined as the point where $\tau_{w}=0$. The surface streamline nearest to the wall leaves the body and the boundary layer is said to separate as seen in Figure 1-1.

\subsection{Laminar Separation Bubbles}

Another significant aspect here is the formation of laminar separation bubbles. The separated flow forms a shear layer which is highly unstable and transition to turbulence occurs. Once this takes place, the turbulent shear stresses begin to energize the shear layer by entraining fluid from the outer stream. The redistribution of energy from the higher momentum outer flow brings the layer closer to the surface and can subsequently reattach the separated layer downstream, this time as a turbulent boundary layer. The region between the separation point and reattachment point is referred to as the separation bubble. 
The conditions that trigger the formation of a laminar separation bubble, i.e. the conditions causing the occurrence of separation, transition and reattachment without the transition to turbulence, depend on the Reynolds number, the pressure distribution, the surface curvature, the surface roughness, the free stream turbulence as well as other environmental factors. For high Reynolds numbers, transition typically takes place ahead of the separation point. For moderate Reynolds numbers, separation takes place before transition. Figure 1-1 shows the formation of a laminar separation bubble, Gad-El Hak (2).

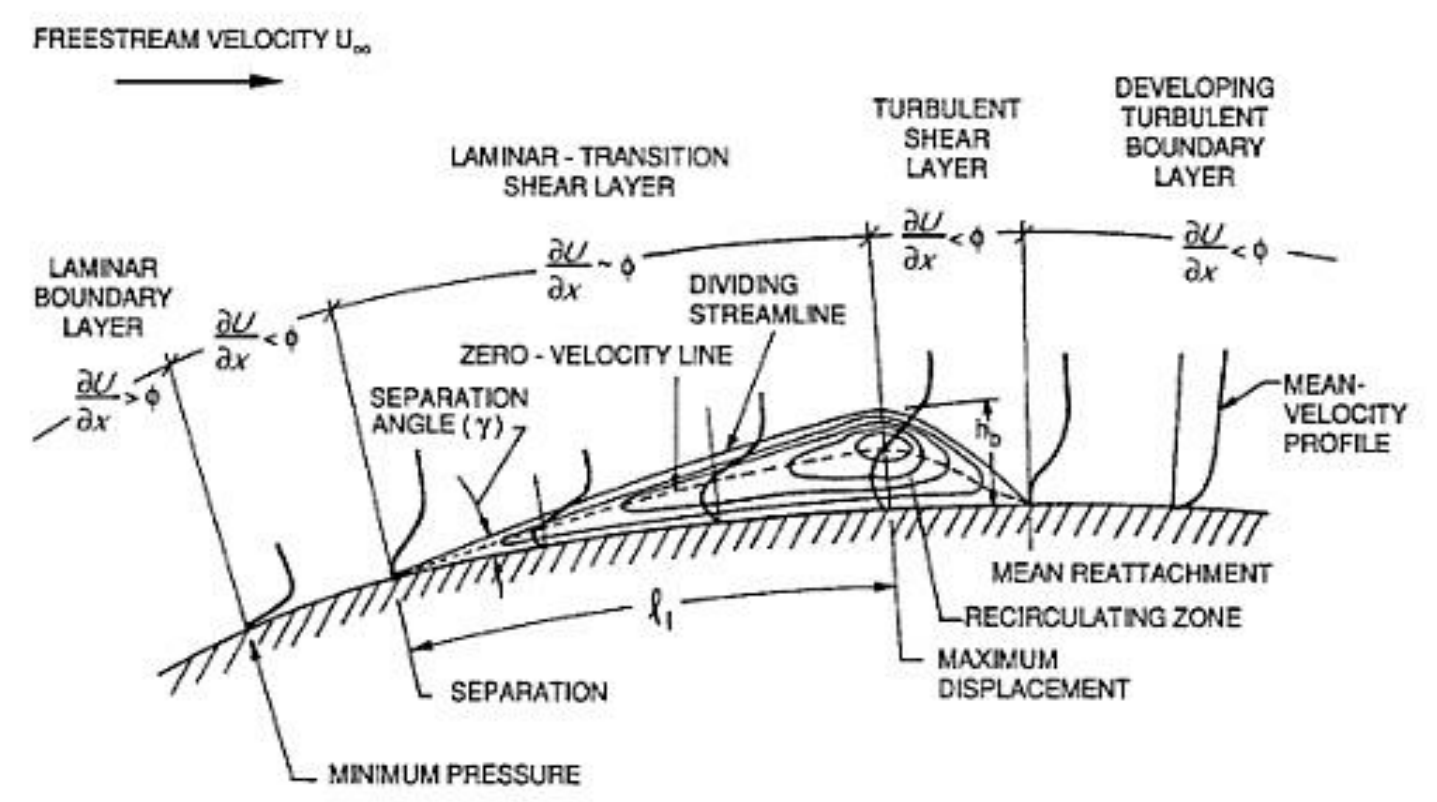

Figure 1.1 Sketch of a laminar separation bubble (2)

Basically there are two types of separation bubbles - short bubble and long bubble. The short separation bubble generally has a length of the order of a few percent of the chord. The nature of the bubble is greatly affected by the Reynolds number and the angle of attack. The existence of the bubble however, does not significantly alter the global lift and drag characteristics. The separation bubble's length is inversely proportional to the Reynolds number; i.e., as the Reynolds 
number is increased, there is a stream-wise contraction of the bubble size. Also as the angle of attack is increased, the bubble tends to move forward. At certain values of Reynolds number and angle of attack, the turbulent mixing and entrainment processes can no longer increase the negative pressure coefficient sufficient enough for the reattachment to take place. At this point, the short bubble is said to burst forming a long bubble. A long bubble tends to increase in length as the angle of attack is increased. The long bubble may reattach further downstream or not reattach at all. For chord Reynolds numbers below $5 \times 10^{4}$, the chord is generally too short for the separated boundary layer to reattach. At a chord Reynolds number of approximately $7 \times 10^{4}$, the chord is long enough for the separated boundary layer to reattach and form the bubble. The bursting phenomenon causes an abrupt loss of lift and increase in pressure drag. This gives rise to the onset of stall.

\subsection{Stall}

Stall is the reduction of the lift that is observed as the angle of attack increases and flow separation occurs. This generally occurs when the critical angle of attack of the airfoil is exceeded. Stall is of three types - leading edge stall, thin airfoil stall and trailing edge stall. As angle of attack is increased for the first type, the separation occurs right at the leading edge. The flow typically attaches a short distance downstream creating a separation bubble. As angle of attack is further increased or Reynolds number is decreased, the reattachment point moves downstream further reducing lift. The reattachment may not occur due to short chord length. These lead to an abrupt loss of lift. This phenomenon is known as the leading edge stall. When an airfoil has a low thickness ratio or a sharp leading edge, another type of stall, known as the thin airfoil stall, occurs. The leading edge stall is basically due to abrupt flow separation near the leading edge without subsequent reattachment whereas the thin airfoil stall is preceded by flow 
separation at the leading edge with reattachment at a point which moves rearward progressively with increase in angle of attack, McCullough and Gault (3). The trailing edge stall is associated with the flow separation occurring at the trailing edge with the separation point of the turbulent boundary layer moving forward from the trailing edge as the angle of attack increases, and is generally observed for thicker airfoil sections. A significant phenomenon over the NACA 0012 airfoil in the low Reynolds number is the leading edge stall. In order to avoid the consequences of this, such as abrupt drop of lift, increase in pressure drag, flow control is necessary.

\subsection{Flow Control}

Control of flow separation, is one of the kinds of boundary layer control and is probably the oldest and most economically important one. It is of immense importance to the performance of air, land and sea vehicles; turbo machines; diffusers and a variety of other systems involving fluid flow, Gad-El Hak (2). Postponement of separation reduces form drag, delays stall, enhances lift and improves pressure recovery. Typical applications of flow separation control include effective low-Reynolds number airfoils for remotely piloted vehicles, propellers, windmills, helicopters, improved axial flow compressors, efficient inlets and diffusers. Some of the benefits of flow separation control are increased maximum lift coefficient for greater payload, reduced engine power and noise at takeoff, shorter runways, and reduced approach speed, super maneuverability or birdlike flight, efficient and effective stall or spin control, reduced drag on missiles, automobiles, ships and helicopters etc. As examples of estimated benefits, a 5\% improvement in the lift coefficient would allow a 25\% payload increase as well as, mitigation of stall and spin accidents Gad-El Hak (2). Application of flow separation control over trucks results in tractor-trailer truck drag reductions which could save in excess of 50 million barrels of oil per year Gad-El Hak (2). 
Over an airfoil, the main goals of general flow control are to increase performance by maintaining lift, reducing drag and improving the stall characteristics. The main objectives include delaying or eliminating flow separation, delaying boundary layer transition and reducing skin friction drag. These improve flight controllability and maneuverability, and provide significant savings in overall fuel consumption.

\subsection{Methods of Flow Control}

There are many active and passive methods of flow control. One such technique has been to add momentum to the near wall region either actively (e.g. tangential blowing or wall jets) or passively (e.g. boundary layer tripping, turbulence enhancement or vortex generators of various scales), Gad-El Hak(2). Many conceptual solutions to the problem of flow control have been proposed. There are several reasons why most of them were not implemented. Some serious problems associated with various types of flow control include parasitic device drag, energy consumption, system weight, volume, complexity, reliability, cost and performance sensitivity to body attitude or orientation. Some systems have higher power usage requirements than power savings, resulting in a net energy loss as in the case of a boundary layer suction flow control system. The system results in reduction of aerodynamic drag but the energy resources required to operate the system exceed the energy savings obtained by application of the system. So the overall system efficiency is reduced. Some systems have high cost and are highly complex. All these issues have made many flow control systems impractical and have ruled out their application in the real-world environment. So extensive research is being done to narrow down to an ideal, most efficient flow control system which improves the overall aerodynamic efficiency of the flight vehicles. 
There are several options for flow control. Surface parameters like shape, roughness, curvature, rigid wall motion, temperature and porosity can influence the flow. Heating and cooling of the surface can influence the flow through density and viscosity changes, changing the transition Reynolds number. A porous wall can result in mass transfer. Near the wall, the shape of the velocity profile can be influenced by suction or injection of fluids which in turn affect the boundary layer characteristics of transition and separation. Additives such as polymers, surfactants, particles, micro bubbles and droplets can also be injected. All these are part of the near wall flow control techniques. Bombarding a shear layer by acoustic waves, magneto and electro hydrodynamic body forces are examples of flow control strategies applied away from the wall (2).

The flow control devices can be active or passive. Passive devices do not require auxiliary power whereas the active ones require energy expenditure. The active devices are further classified into predetermined and reactive categories. The predetermined control has energy applied steadily or unsteadily without regard to particular state of the flow. Reactive control has the input power continuously adjusted based on feedback from the flow; i.e. power is altered based on the state of the flow (4).

\subsection{Flow Control Using Dynamic Roughness (DR)}

Several methods for flow control have been tried over the past few years. Active methods such as blowing, suction and synthetic jets were some of the previously tried methods. Another method of flow control is periodic excitation. Actuators are considered to be devices that interact with flow hydrodynamically to produce oscillatory addition of momentum with or without superimposed mass flux as defined by Greenblatt and Wygnanski (4). The main objective of this research is to investigate the 3-D dynamic roughness effects on flow over a NACA 0012 airfoil 
with focus on controlling flow separation in the low Reynolds number regime of $10^{4}-10^{5}$. Flow control is achieved by means of dynamic surface roughness elements on an airfoil, placed near the leading edge, aimed at increasing the efficiency by suppressing the effects of leading edge separation, like leading edge stall, by delaying or totally eliminating flow separation. These surface perturbations can be 2D or 3D time-dependent humps on the surface of the airfoil that are on the scale of the local boundary layer thickness which have an unsteady motion. However in this study, 3D simulations have been performed. The amplitude and frequency are tunable based on flow conditions, Huebsch (5). This method aims to provide a means to modify the instantaneous and mean velocity profile near the wall and thereby control the local state of the boundary layer. This can lead to the suppression of adverse effects of flow separation.

Some explanations for this flow control mechanism are alteration of flow instabilities, creation of hairpin type vortices in the viscous sub layers of the boundary layer which enhance mixing and entrainment, creation of artificial Reynolds stresses and favorable alterations of the pressure gradient Huebsch et al. (6). These roughness elements add energy to the energy deficit separating boundary layer. It thus eliminates or delays separation. If size of the dynamic roughness elements is on the scale of the approaching boundary layer and if they are introduced just upstream of the separation point, the state of the approaching boundary layer will be altered prior to it reaching its natural separation point as defined by Huebsch (5). This altered state is totally different from the laminar boundary layer which tends towards separation, but the dynamic roughness does not act like a boundary layer trip device. Dynamic roughness has the potential to turn out as an ideal flow control system which doesn't face the shortcomings faced by many other proposed flow control systems. Some of the proposed systems have a higher power usage than power savings and also have a high cost. 
The current research focuses on the numerical study of the dynamic roughness in controlling flow separation (leading edge stall) over a NACA 0012 airfoil at low Reynolds numbers. This airfoil has been chosen in simulations in order to compare it with experimental PIV findings for flow over the same. Owing to the highly unsteady nature of flow in this exercise, large eddy simulations have been performed using the commercial CFD code Fluent in this work. User defined functions (UDFs) have been written to simulate the motion of the dynamic surface roughness elements.

\subsection{Experimental and Numerical Evidence}

There have been multiple past studies on the ability of dynamic roughness to control flow separation. The ability of dynamic roughness to control the flow has been clearly demonstrated experimentally by Grager et al. (7) with the help of PIV. It has been clearly shown that the leading edge stall has been suppressed by dynamic roughness. The current study is a numerical extension to the above study, with large eddy simulations being performed using Fluent to study the control of leading edge stall with dynamic roughness. Experimental and numerical studies on the effects of dynamic roughness on separation for flow control have been performed by Gall (8) at low Reynolds numbers. These have successfully demonstrated the significant impact of dynamic roughness by eliminating the separation bubble and reattaching the flow. Dynamic roughness as a means of leading edge separation control has been discussed with experimental and numerical studies by Huebsch et al. (6). Also flow visualization by Jakkali et al. (9) clearly shows the effect of dynamic roughness in reattaching the flow and suppressing stall at various Reynolds numbers. Experimental research has been done mainly with 3D humps whereas most of the numerical simulations have dealt with $2 \mathrm{D}$ simulations although there were numerical studies with 3D humps. Those numerical studies were mainly limited to RANS simulations 
which have several shortcomings while dealing with unsteady flows. The current study has been performed with 3D dynamic roughness elements using large eddy simulations which are efficient when the flow is highly unsteady as in this study. The results have compared to experimental results from Grager (10). 


\section{Numerical Formulation}

The flow physics for flow control using dynamic roughness involve mixing and entrainment of higher momentum fluid with the one with lower energy giving it a turbulent nature. Also in the low Reynolds number range, the flow will be laminar, transitional and turbulent along the wing. So there is a great need to effectively capture all the three modes.

Almost all types of flows in the universe have an inherent turbulent nature associated with them. Flow of water and weather patterns are a few such examples where turbulence can play a dominant role. It is observed in experiments that below a certain value of Reynolds number called the critical Reynolds number, flow is smooth and there is order in the flow. This regime is called laminar flow. For flows with values above the critical Reynolds number, the flow behavior is random and chaotic. This regime is called turbulent flow. It is characterized by chaotic fluctuations in momentum and energy. Turbulent flows are characterized by rotational flow structures called turbulent eddies with a wide range of length scales. These eddying motions cause effective mixing and dissipation of mass, momentum and heat. Kinetic energy, extracted from the mean motion, is transported from the larger eddies to the smaller eddies and this forms the energy cascade. This energy is then dissipated by the smallest eddies as heat.

The change from laminar to turbulent state due to instability of the laminar flow is known as transition. The transition process generally involves amplification of initially small disturbances, creation of areas with rotational structures, formation of intense small-scale motions, and growth and merger of these motions to fully turbulent flows. The transition to turbulence is influenced by factors such as pressure gradient, disturbance levels, wall roughness and heat transfer. The adverse pressure gradient on an airfoil separates the laminar boundary layer. The separated shear 
layer is unstable, and based on this instability and the velocity distributions, the laminar boundary layer transitions to a turbulent state. After transition, the turbulent shear stresses energize this layer and entrain fluid from the outer stream which has a higher momentum. Due to this redistribution of energy, reattachment occurs. This is basically the process behind the creation of a separation bubble. In the case of low Reynolds number or high angles of attack, the separated layer may not reattach and the airfoil goes into complete stall conditions. Numerical modelling of this flow phenomenon is itself difficult as all the three types of flow behaviors laminar, transition and turbulence have to be taken into account and controlling this by DR is much more difficult.

Boutilier and Yarusevych (11) have described the transition phenomenon over airfoils at low Reynolds numbers in detail. There are many questions that need to be answered in this area of interest. Modeling turbulence plays a key role in defining the physics behind various topics associated with turbulent flows. All the past work which has been done was basically laminar studies aimed at introducing and demonstrating the effectiveness of dynamic roughness as a novel flow control mechanism. In order to capture and study the complete flow physics effectively, turbulence modelling is necessary. There is no one particular turbulence model which is effective to study all physical process. It is more area specific. Several successful new models have been developed and quite a lot have been put to great use. Research is still being carried out for more efficient and precise models which can study and address questions related to turbulence in depth.

\subsection{Modeling Turbulence}

Turbulence has a wide range of length and time scales which interact in a dynamic and complex manner. A great deal of extensive research has been undertaken and is still underway in 
developing methods that capture and define physics associated with turbulence and its effects. All the methods may be classified into three main categories -Direct Numerical Simulation (DNS), turbulence models for Reynolds Averaged Navier - Stokes (RANS) equations and Large Eddy Simulation (LES).

\subsubsection{Direct Numerical Simulation}

Direct numerical simulations (DNS) compute mean flow and all turbulent velocity fluctuations down to the very smallest eddy scales directly. These are simulations without any turbulence models and can be termed numerical experiments. The whole range of spatial and temporal scales must be resolved, i.e. from the smallest dissipative scales - Kolmogorov length scales to the integral length scales which contain most of the turbulent energy. The unsteady Navier Stokes equations are solved on spatial grids which are extremely fine and the with time steps very small (smaller than $10^{-7} \mathrm{~s}$ ) to resolve periods of fast fluctuations. The number of operations in these simulations grows as $\mathrm{Re}^{3}$. The computational cost of DNS is very high even at low Reynolds numbers. Though costly, from a DNS it is possible to extract information that is difficult or impossible to obtain in a laboratory. So it can be a powerful tool to help understand the physics of turbulence. For the Reynolds numbers encountered in industrial applications, the computational resources required by a DNS are not available or may not be sufficient.

\subsubsection{Reynolds Averaged Navier Stokes (RANS) Models}

Turbulent flows can be characterized in terms of the mean values of flow properties and some statistical properties of their fluctuations. This is known as the Reynolds decomposition. The Navier Stokes equations are time averaged and extra terms appear in the time averaged flow equations due to interactions between various turbulent fluctuations. For instance, the original momentum equations are: 


$$
\begin{aligned}
& \frac{\partial u}{\partial t}+\operatorname{div}(u \mathrm{u})=-\frac{1}{\rho} \frac{\partial p}{\partial x}+v \operatorname{div}(\operatorname{grad}(\mathrm{u})) \\
& (\mathrm{X}-\text { momentum equation }) \\
& \frac{\partial v}{\partial t}+\operatorname{div}(v \mathrm{u})=-\frac{1}{\rho} \frac{\partial p}{\partial \mathrm{y}}+v \operatorname{div}(\operatorname{grad}(v)) \\
& (\mathrm{Y}-\text { momentum equation }) \\
& \frac{\partial w}{\partial t}+\operatorname{div}(w \mathrm{u})=-\frac{1}{\rho} \frac{\partial p}{\partial z}+v \operatorname{div}(\operatorname{grad}(w)) \\
& (\mathrm{Z}-\text { momentum equation })
\end{aligned}
$$

The time-averaged momentum equations are:

$\frac{\partial U}{\partial t}+\operatorname{div}(U U)=-\frac{1}{\rho} \frac{\partial P}{\partial x}+v \operatorname{div}(\operatorname{grad}(U))+\frac{1}{\rho}\left[\frac{\partial\left(-\rho \overline{u^{\prime 2}}\right)}{\partial x}+\frac{\partial\left(-\rho \overline{u^{\prime} v^{\prime}}\right)}{\partial y}+\frac{\partial\left(-\rho \overline{u^{\prime} w^{\prime}}\right)}{\partial z}\right]$

(Time-averaged $\mathrm{X}$ - momentum equation)

$\frac{\partial V}{\partial t}+\operatorname{div}(V \mathrm{U})=-\frac{1}{\rho} \frac{\partial P}{\partial y}+v \operatorname{div}(\operatorname{grad}(V))+\frac{1}{\rho}\left[\frac{\partial\left(-\rho \overline{u^{\prime} v^{\prime}}\right)}{\partial x}+\frac{\partial\left(-\rho \overline{v^{\prime 2}}\right)}{\partial y}+\frac{\partial\left(-\rho \overline{v^{\prime} w^{\prime}}\right)}{\partial z}\right]$

(Time-averaged $\mathrm{Y}$ - momentum equation)

$$
\frac{\partial W}{\partial t}+\operatorname{div}(W \mathrm{U})=-\frac{1}{\rho} \frac{\partial P}{\partial z}+v \operatorname{div}(\operatorname{grad}(W))+\frac{1}{\rho}\left[\frac{\partial\left(-\rho \overline{u^{\prime} w^{\prime}}\right)}{\partial x}+\frac{\partial\left(-\rho \overline{v^{\prime} w^{\prime}}\right)}{\partial y}+\frac{\partial\left(-\rho \overline{v^{\prime} w^{\prime}}\right)}{\partial z}\right]
$$

(Time-averaged $\mathrm{Z}$ - momentum equation) 
The extra turbulent stresses appearing in the above equations (2.4-2.6) are called Reynolds stresses and are comprised of normal stresses and shear stresses. These contain averages of the squared velocity fluctuations and hence are always non-zero. The above three equations are called the Reynolds-averaged Navier-Stokes equations (RANS). Here the lower case components are instantaneous, the upper case ones are the mean velocity components and the over bar represents averaging. These Reynolds stresses have to be related to the mean motion itself before the equations are solved since the number of unknowns and equations must be equal. The absence of these additional equations is referred to as the Turbulence Closure problem. To compute turbulent flows with RANS, turbulence models need to be used to predict the Reynolds stresses and the scalar transport terms and close the system of mean flow equations. The RANS turbulence models are classified based on the number of additional transport equations that need to be solved along with RANS flow equations. Some of them are listed in Table 2-1.

Table 2-1 List of RANS models

\begin{tabular}{|c|c|}
\hline No. of extra transport equations & Name \\
\hline Zero & Mixing length model \\
\hline One & Spallart - Allmaras model \\
\hline Two & $k-\epsilon$ model, $k-\omega$ model, Algebraic stress model \\
\hline Seven & Reynolds stress model \\
\hline
\end{tabular}

RANS models all scales of turbulence. The computing resources required for these simulations are modest. Also they are quicker in terms of time. These are reasonably accurate in some cases and are generally widely in industry. However RANS is inaccurate in many flows. 


\subsubsection{Large Eddy Simulation}

Large Eddy Simulation (LES) is an intermediate method of turbulence calculations which deals mainly with the large eddies. This involves filtering of the unsteady Navier-Stokes equations prior to computations. Large eddies are directly calculated and smaller ones below the filter limit are modeled. The effects of the smallest, unresolved eddies on the resolved flow are included by means of a sub-grid scale model. The computational resources required for LES are large but comparatively less than a DNS. The method starts off with the selection of filtering function and a cutoff width to resolve all eddies with length scale greater than the cutoff width. Then a spatial filtering operation is done on the unsteady flow equations. During this process, the detailed information about the smaller filtered out eddies is destroyed. This and interaction effects between larger eddies and smaller eddies gives rise to the sub-grid scale stresses, SGS. Their effect on the resolved flow must be described by a SGS model. In the finite volume method, the time-dependent space filtered flow equations are solved on a grid of control volumes along with the SGS model for unresolved stresses.

\subsection{Need to go for a Large Eddy Simulation}

Research is still being undertaken to develop a general- purpose turbulence model to suite a wide range practical applications despite the development of a large number of RANS models. The main driving element for this is the difference in the behavior of large and small eddies. Smaller eddies have a universal behavior and are isotropic at least for flows at high Reynolds numbers. Large eddies interact with the mean flow and extract energy from it. These are anisotropic and largely depend on the geometry of the problem domain, boundary conditions and body forces. While using RANS, all eddies must be described by a single turbulence model but the problem dependence of large eddies complicates the search for widely applicable models. So LES takes 
into account this issue by resolving the larger eddies for each specific flow with a time dependent simulation and universal behavior of the smaller eddies is modeled.

For numerical studies on an airfoil, unsteady RANS (URANS) can predict regular vortex shedding at the largest scale. The difference between RANS and URANS is the presence of an additional unsteady term in the momentum equation. However URANS falls short of capturing the remaining scales. URANS is incapable of capturing internally induced fluctuations of the flow field and hence cannot replace LES when turbulent mixing needs to be taken into account. URANS has the inability to model the physics of large scale structures in transporting momentum and scalars. Figure 2-1 is a clear indication of the failure of URANS models, where it has been compared to LES. The airfoil at this angle of attack physically produces vortices that are shed from the surface. LES was able to capture dominant large scale features of this flow while URANS could not.

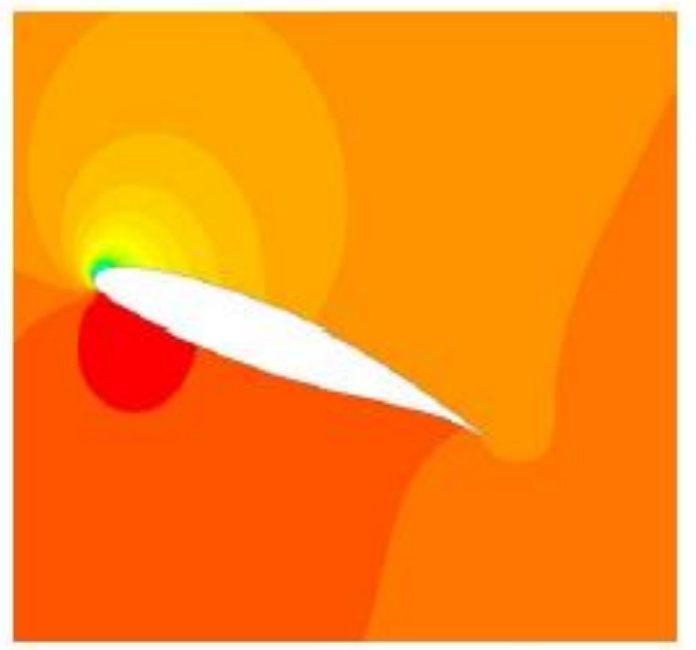

Unsteady RANS with $k$-w SST model

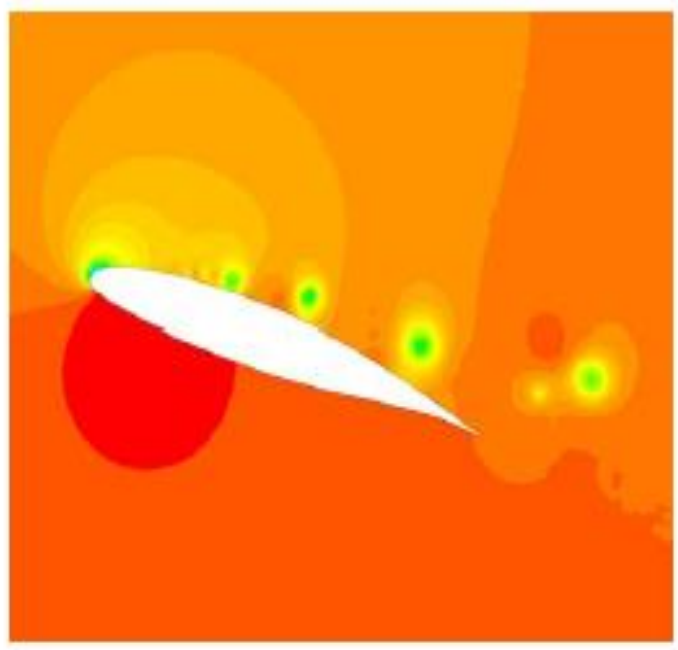

LES

Figure 2.1 RANS and LES comparison of velocity contours, Kim (12) 
Weber and Ducros (13) have discussed the differences in URANS simulations and LES for turbulent flows over an airfoil. They have stated that LES is the best method for modeling highly unsteady flows. Piomelli (14) has laid out in detail the potential of LES in handling unsteady flows. He gives a detailed account of LES and its capabilities and challenges. LES has been successfully used by many researchers to study the flow control over airfoils. Almutairi et al. (15) have studied the behavior of a laminar separation bubble near stall. Kojima et al. (16) performed a detailed numerical study with LES for flows over thick and thin airfoils at low Reynolds numbers. The separation phenomenon has been captured and studied well. Numerical studies of Roberts and Yaras (17) show that LES accurately captures the transition process in the separated shear layer. Hence LES is an ideal choice to study the process of flow control over an airfoil given its highly unsteady nature with flow separation at angle of attack.

\subsection{Filtering Operation}

LES is based on a filtering operation to separate large scales from the small scales. The governing equations are thus obtained by filtering the time dependent Navier Stokes equations. A filtered variable (denoted by an over bar) is defined as:

$\bar{\phi}(\mathrm{x})=\int_{D} \phi\left(x^{\prime}\right) G\left(x, x^{\prime}\right) d x^{\prime}$

$\mathrm{D}$ is the domain and $\mathrm{G}$ is the filter function that determines the scale of the resolved eddies. The application of filtering operation gives the filtered equations of motion. For an incompressible flow, they are:

$\frac{\partial \bar{u}_{i}}{\partial \bar{x}_{i}}=0$ 


$$
\frac{\partial \bar{u}_{i}}{\partial t}+\frac{\partial}{\partial x_{j}}\left(\bar{u}_{i} \bar{u}_{j}\right)=-\frac{1}{\rho} \frac{\partial \bar{p}}{\partial x_{i}}-\frac{\partial \tau_{i j}}{\partial x_{j}}+v \frac{\partial^{2} \bar{u}_{i}}{\partial x_{j} \partial x_{j}}
$$

The evolution of large energy carrying scales is governed by these equations. The effect of small scales appears through a subgrid scale (SGS) stress term, $\tau_{i j}$.

In Fluent, the finite volume discretization itself implicitly provides the filtering operation:

$\bar{\phi}(\mathrm{x})=\int_{v} \phi\left(x^{\prime}\right) d x^{\prime}\left(x^{\prime} \in v\right)$

where $\mathrm{V}$ is the volume of the computational cell. The filter function here is:

$G\left(x, x^{\prime}\right)=\left\{\begin{array}{l}\frac{1}{V}, x^{\prime} \in v \\ 0, x^{\prime} \text { otherwise }\end{array}\right.$

The subgrid scale stress term is given by: $\tau_{i j}={\overline{u_{\imath} u_{\jmath}}}_{-} \bar{u}_{i} \bar{u}_{j}$.

The subgrid scale velocity is given by:

$u_{i}^{\prime}=u_{i}-\bar{u}_{i}$

The SGS stresses can be decomposed into three parts:

$\tau_{i j}=\overline{u_{\imath} u_{j}}-\bar{u}_{i} \bar{u}_{j}=L_{i j}+C_{i j}+R_{i j}$

where $L_{i j}=\overline{\overline{u_{i} u_{j}}}-\overline{u_{i}} \overline{u_{j}}$ are the Leonard stresses, $C_{i j}=\overline{\overline{u_{\imath} u^{\prime}}}+\overline{\overline{u_{\jmath}^{\prime}} \overline{u_{l}}}$ are the cross terms and $R_{i j}=\overline{u^{\prime}{ }_{l}^{\prime}{ }_{j}}$ are the SGS Reynolds stresses. The interactions between resolved scales that result in subgrid-scale contributions are represented by the Leonard stresses. They can be directly obtained and are the aliasing errors when a sharp cutoff filter is used. Cross terms represent 
interactions between resolved and unresolved scales. SGS Reynolds stresses represent interactions between small, unresolved scales. The decomposition is rarely done.

\subsection{Subgrid Scale Models}

The subgrid scale stresses which arise due to the filtering operation are unknown and hence require modeling. In LES, dissipative scales are not resolved. The main function of the subgrid scale model is to drain energy from the resolved scales and mimic the termination of the energy cascade at the smallest scales. The subgrid scale models calculate the subgrid scale turbulent stresses from:

$\tau_{i j}-\frac{1}{3} \tau_{k k} \delta_{i j}=-2 \mu_{t} \bar{S}_{i j}$

Here $\mu_{t}$ is the subgrid scale turbulent viscosity. $\tau_{k k}$, the isotropic part of the subgrid scale stresses, is not modeled and is added to the static pressure term. By the above equation, the subgrid scale stresses are related to the strain rate tensor $\bar{S}_{i j}$ which is given by:

$\bar{S}_{i j}=\frac{1}{2}\left(\frac{\partial \bar{u}_{i}}{\partial x_{j}}+\frac{\partial \bar{u}_{j}}{\partial x_{i}}\right)$

There are basically four models for $\mu_{t}$ in FLUENT. They are the Smagorinsky-Lilly model, the dynamic Smagorinsky-Lilly model, the WALE model, and the dynamic kinetic energy subgridscale model.

\subsubsection{Smagorinsky-Lilly Model}

This model was first proposed by Smagorinsky (18). In this model, the eddy viscosity is given by: 
$\mu_{t}=\rho L_{s}^{2}|\bar{S}|$

Here $L_{S}$ is the mixing length for subgrid scales and

$$
|\bar{S}|=\sqrt{2 \bar{S}_{i j} \bar{S}_{i j}}
$$

In FLUENT, $L_{s}$ is calculated as follows:

$L_{s}=\min \left(K d, C_{s} V^{\frac{1}{3}}\right)$

Here $K$ is the von Kármán constant, $d$ is the distance to the closest wall, $C_{S}$ is the Smagorinsky constant and $V$ is the volume of a computational cell.

Lilly calculated a value of 0.17 for $C_{S}$ for homogenous isotropic turbulence in the inertial subrange. However in the presence of shear, near solid boundaries, or in transitional flows, this value has caused excessive damping of large scale fluctuations and must be decreased. $C_{S}$ is not a universal constant. This is a drawback with this model.

\subsubsection{Dynamic Smagorinsky-Lilly Model}

In dynamic models, based on the energy content of the smallest resolved scale, the coefficients of the model are determined as the calculation progresses. Germano, et al. (19) and subsequently Lilly (20) developed a procedure in which the Smagorinsky constant $C_{S}$ is computed based on the information provided by the resolved scales of motion. So one doesn't need to specify the value of $C_{s}$ in advance; instead it is dynamically calculated. The dynamic model allows the Smagorinsky constant to vary in space and time. It is calculated locally in each time step based 
on two filterings of the flow variables which are denoted by "_" and " $"$ ". These are the grid and test filters respectively. The test filter width is larger than the grid filter width.

Filtering with the grid filter results in equations with $\tau_{i j}$ given by:

$\tau_{i j}=\overline{u_{\imath} u_{\jmath}}-\bar{u}_{i} \bar{u}_{j}$

Filtering again with the test filter yields a similar set of equations but with a different subgridscale stress term.

$T_{i j}=\widetilde{\overline{u_{\imath} u_{\jmath}}}-\tilde{\bar{u}}_{i} \tilde{\bar{u}}_{j}$

The two subgrid scale stresses are related by the Germano identity and the resolved turbulent stress $L_{i j}$ is defined as:

$L_{i j}=T_{i j}-\tilde{\tau}_{i j}$

where $L_{i j}=\overline{\bar{u}}_{\imath} \bar{u}_{j}-\tilde{\bar{u}}_{i} \tilde{\bar{u}}_{j}$

The resolved turbulent stresses are representative of the contribution to Reynolds stresses by scales of intermediate length between grid filter width and test filter width. The Germano identity is used to calculate dynamic local values for $C_{S}$ by applying the Smagorinsky model to both $T_{i j}$ and $\tau_{i j}$. The anisotropic part of $L_{i j}$ is represented as:

$L_{i j}-\frac{\delta_{i j} L_{k k}}{3}=-2 C_{S} M_{i j}$

where 
$M_{i j}=(\widetilde{\Delta})^{2}|\tilde{\bar{S}}| \tilde{\bar{S}}_{i j}-(\bar{\Delta})^{2} \mid \overline{\bar{S} \mid \bar{S}_{l j}}$

$C_{S}$ is calculated from:

$C^{2}{ }_{s}=-\frac{1}{2} \frac{L_{k l} \bar{S}_{k l}}{M_{m n} \bar{S}_{m n}}$

For stabilization, $C_{s}$ is averaged in the homogenous direction. If this is not possible, local averaging has been used in place of an average in a homogenous direction.

As an alternate solution, Lilly proposed a least squares procedure that is generally taken into account rather than the original calculation of $C_{S}$

$C^{2}{ }_{s}=-\frac{1}{2} \frac{L_{k l} M_{k l}}{M_{m n} M_{m n}}$

Stabilization should be done even in this case.

\subsubsection{Wall-Adapting Local Eddy-Viscosity (WALE) Model}

In the WALE model given by Nicoud and Ducros (21), the eddy viscosity is modeled as follows:

$\mu_{t}=\rho L^{2}{ }_{s} \frac{\left(s_{i j}^{d} s_{i j}^{d}\right)^{3 / 2}}{\left(\bar{s}_{i j} \bar{s}_{i j}\right)^{5 / 2}+\left(s_{i j}^{d} s_{i j}^{d}\right)^{5 / 4}}$

where $L_{s}$ and $S_{i j}^{d}$ are defined as:

$L_{s}=\min \left(K d, C_{w} V^{\frac{1}{3}}\right)$

and 
$S_{i j}^{d}=\frac{1}{2}\left(\bar{g}_{i j}^{2}+\bar{g}_{j i}^{2}\right)-\frac{1}{3} \delta_{i j} \bar{g}_{k k}^{2}$

where

$\bar{g}_{i j}=\frac{\partial \bar{u}_{i}}{\partial x_{j}}$

$C_{w}$ is the WALE constant. The default value in FLUENT is 0.325 . With this spatial operator, the

WALE model is designed to return the correct wall asymptotic $\left(y^{3}\right)$ behavior for wall bounded flows.

\subsubsection{Dynamic Kinetic Energy Subgrid-Scale Model}

The original and dynamic Smagorinsky models are algebraic models in which subgrid-scale stresses are parameterized using resolved velocity scales. The underlying assumption here is the local equilibrium between the transferred energy through the grid filter scale and the dissipation of kinetic energy at small subgrid scales. By taking into account the transport of subgrid-scale turbulence kinetic energy, subgrid-scale turbulence can be modeled better. The model implemented in FLUENT replicates the model proposed by Kim and Menon (22). The subgridscale kinetic energy is defined as:

$k_{s g s}=\frac{1}{2}\left(\overline{u_{k}^{2}}-\bar{u}_{k}^{2}\right)$

The subgrid-scale eddy viscosity, $\mu_{t}$, is computed using $k_{s g s}$ as follows:

$\mu_{t}=C_{k} k_{s g s}^{1 / 2} \Delta_{f}$

Here $\Delta_{f}$ is the filter size computed from 


$$
\Delta_{f}=V^{1 / 3}
$$

The subgrid-scale stresses can be written as:

$\tau_{i j}-\frac{2}{3} k_{s g s} \delta_{i j}=-2 C_{k} k_{s g s}^{1 / 2} \Delta_{f} \bar{S}_{i j}$

$k_{s g s}$ is obtained by solving its transport equation:

$\frac{\partial \bar{k}_{s g s}}{\partial t}+\frac{\partial \bar{u}_{j} \bar{k}_{s g s}}{\partial x_{j}}=-\tau_{i j} \frac{\partial \bar{u}_{i}}{\partial x_{j}}-C_{\in} \frac{k_{s g s}^{3 / 2}}{\Delta_{f}}+\frac{\partial}{\partial x_{j}}\left(\frac{\mu_{t}}{\sigma_{k}} \frac{\partial k_{s g s}}{\partial x_{j}}\right)$

The model constants $C_{k}$ and $C_{\in}$ are determined dynamically. The value of $\sigma_{k}$ is hardwired to 1.0.

For the current study, the Dynamic Kinetic Energy Subgrid-Scale model has been used. The advantage this model has over the other models is that it is dynamic in nature. Also, since the flow control physics are based on turbulent kinetic energy transfer, this model is expected to be well suited for the needs of this study. The reason goes back to the energy cascade where the vortex stretching aids the energy transfer. Also physics behind DR involves creation of vortices which result in mixing and entrainment. The subgrid scale turbulence can be better modeled since this model takes into account the transport of subgrid-scale turbulent kinetic energy.

Also (23), (24), (25), (26) and (27) have been used for reference in this study for further understanding and guidance. 


\section{CFD Setup}

A numerical study has been carried out using the commercial CFD package Ansys FLUENT. FLUENT 13.0 version has been used in this study. A parallel processing approach has been used in this study in which the continuous domain is subdivided into smaller domains. This helps in reducing the computational time of the simulation by several days. The current work has been done on the WVU HPC - MOUNTAINEER (28) cluster. The mesh for this work has been developed using Ansys ICEM CFD, a powerful mesh generation software compared to GAMBIT.

\subsection{Mesh}

The current study deals with flow control over a NACA 0012 airfoil. The basic geometry i.e. the airfoil curve has been created using SOLIDWORKS software. This has then been imported into Ansys ICEM and the domain around the airfoil has been created using ICEM. The chord length, $\mathrm{c}$ is $0.15 \mathrm{~m}$. Figure $3-1$ gives the details of the geometry of the domain which has been used for the present study. The lengths have been specified in terms of chord length, c. The airfoil is inclined at an angle of $14^{\circ}$. Generally in most of the numerical studies for airfoils inclined at a certain angle, a perfectly horizontal airfoil (inclined at $0^{\circ}$ ) is used and a mesh is created using that geometry. Angle of inclination is brought into picture by specifying velocity components of mean flow at that specific angle. However for the current study, the geometry itself has the airfoil inclined at $14^{\circ}$. This has been done to prevent false diffusion which seeps into most of the cases in which inclined boundary velocity components have been specified. Also it matches the experiment closer. This geometry tries to replicate the wind tunnel studies of Grager (10). The same width has been used but the length is shorter than the wind tunnel in order to reduce the mesh size. The larger the mesh size, the longer is the time taken for simulation. However 
sufficient length has been provided upstream and downstream of the airfoil for the flow to develop.

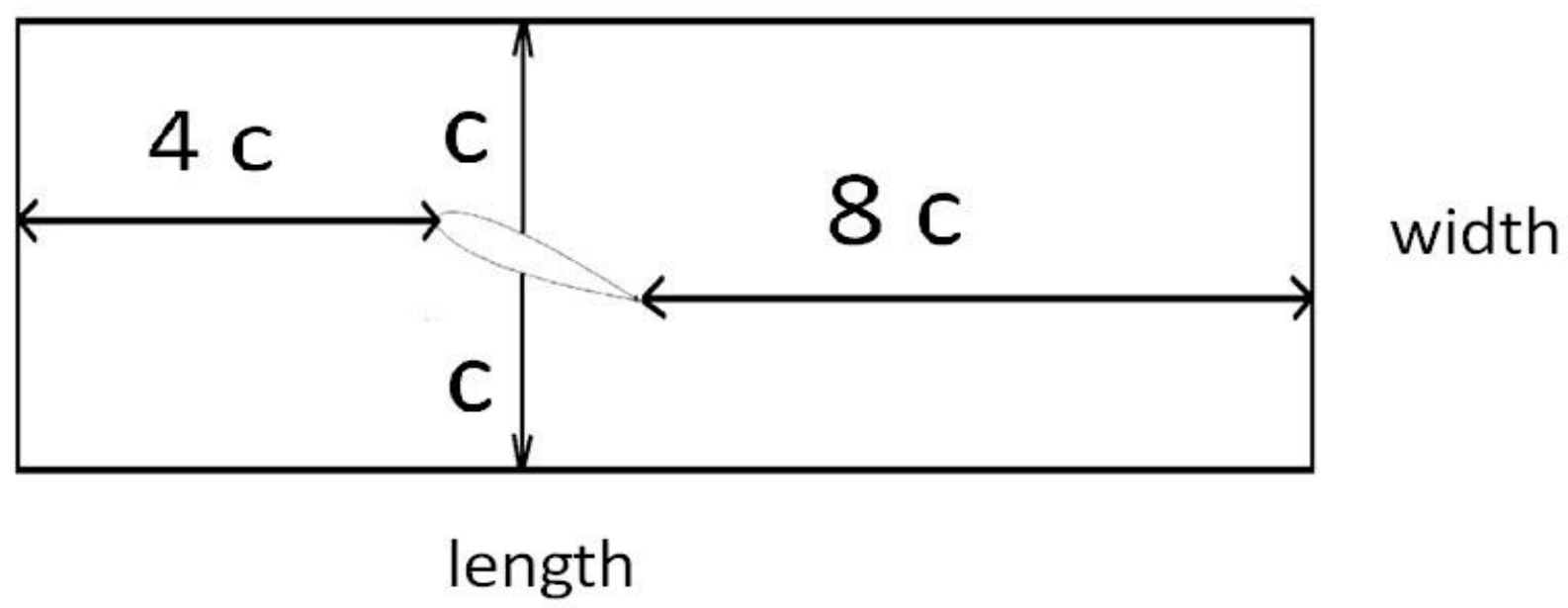

\section{Figure 3.1 Geometry of the domain}

The airfoil has a span of $0.13 \mathrm{C}$ or $13 \%$ of the chord length. The domain also has a span of the same length. There are five rows of staggered humps extending from $0.5 \%$ to $9.5 \%$ of the chord length. A structured mesh has been created for this numerical study. The mesh has been designed according to requirements of a large eddy simulation $\left(\right.$ wall $\left.\mathrm{y}^{+}=1\right)$. The mesh has been designed for numerical study at a Reynolds number of 49000. Figure 3-2 depicts the mesh used in this study.

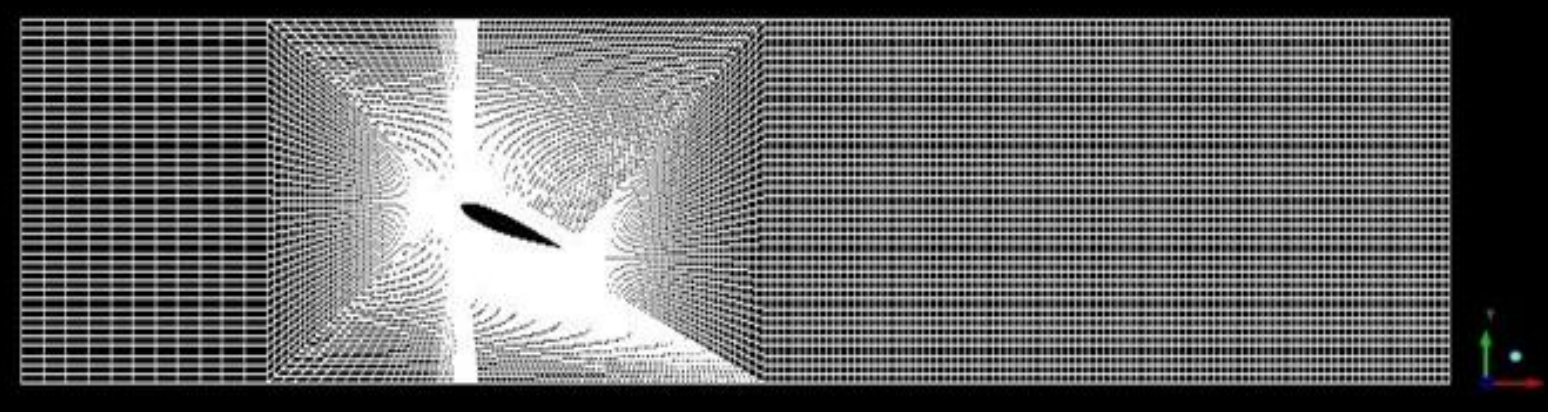

Figure 3.2 Mesh 
There are basically two zones surrounding the airfoil. The inner most zone is the most refined zone. This zone has a height of $50 d s\left(0 \leq \mathrm{y} \leq \mathrm{y}^{+}=50\right)$ corresponding to $y+$ of 1 and has 60 nodes in it. The second zone is slightly less refined than the zone 1 . The height of this zone is $250 d s$ corresponding to $y+$ of 2 with 125 nodes in it. From this zone, the mesh is coarser. Stretching functions have been used from zone 2 onwards and extends away from the airfoil. Figure 3-3 shows the zone 1 and zone 2 together, the finest portion of the mesh and Figure 3-4 shows them separately.

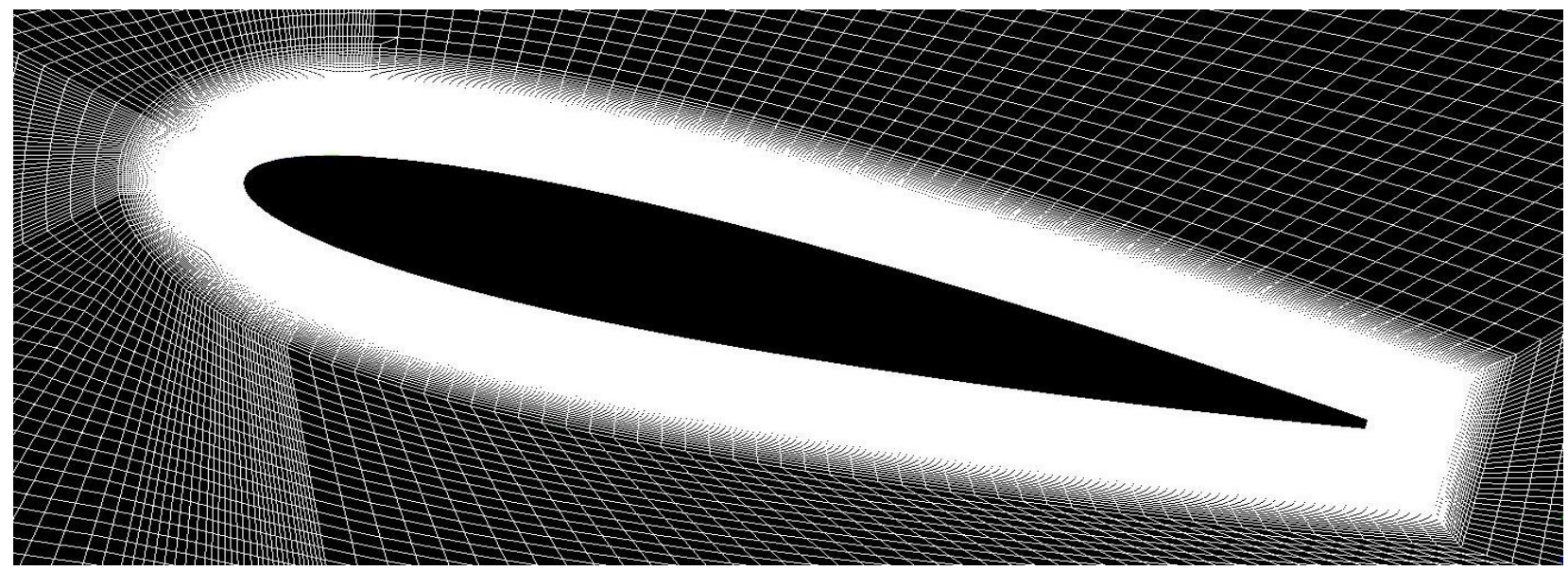

Figure 3.3 Highly refined region of the mesh with zones 1 and 2

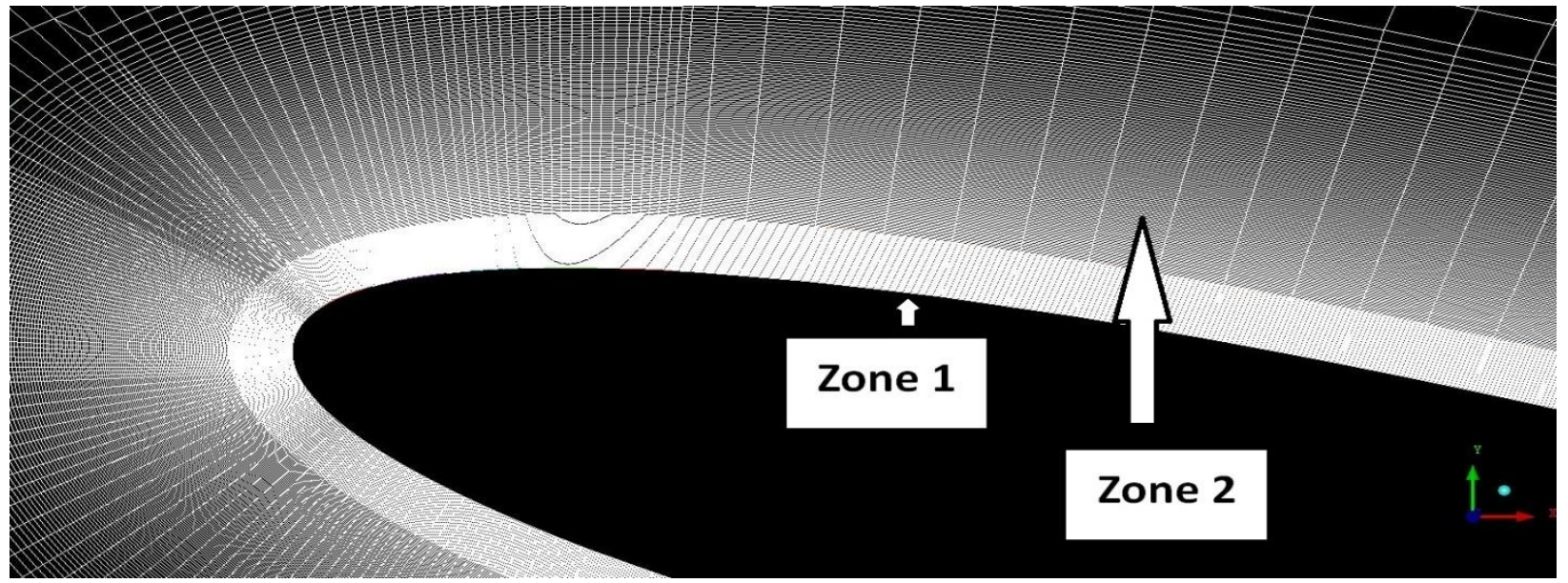

Figure 3.4 Highly refined region of the mesh with zones 1 and 2 (locally enlarged) 
There are 5 ridges which transform into the equally spaced (in respective rows) hump pattern by means of a UDF. Here the term ridge refers to the surface strip or the row. The 3D grid view with the ridges (colored) can be clearly seen in the Figure 3-5.

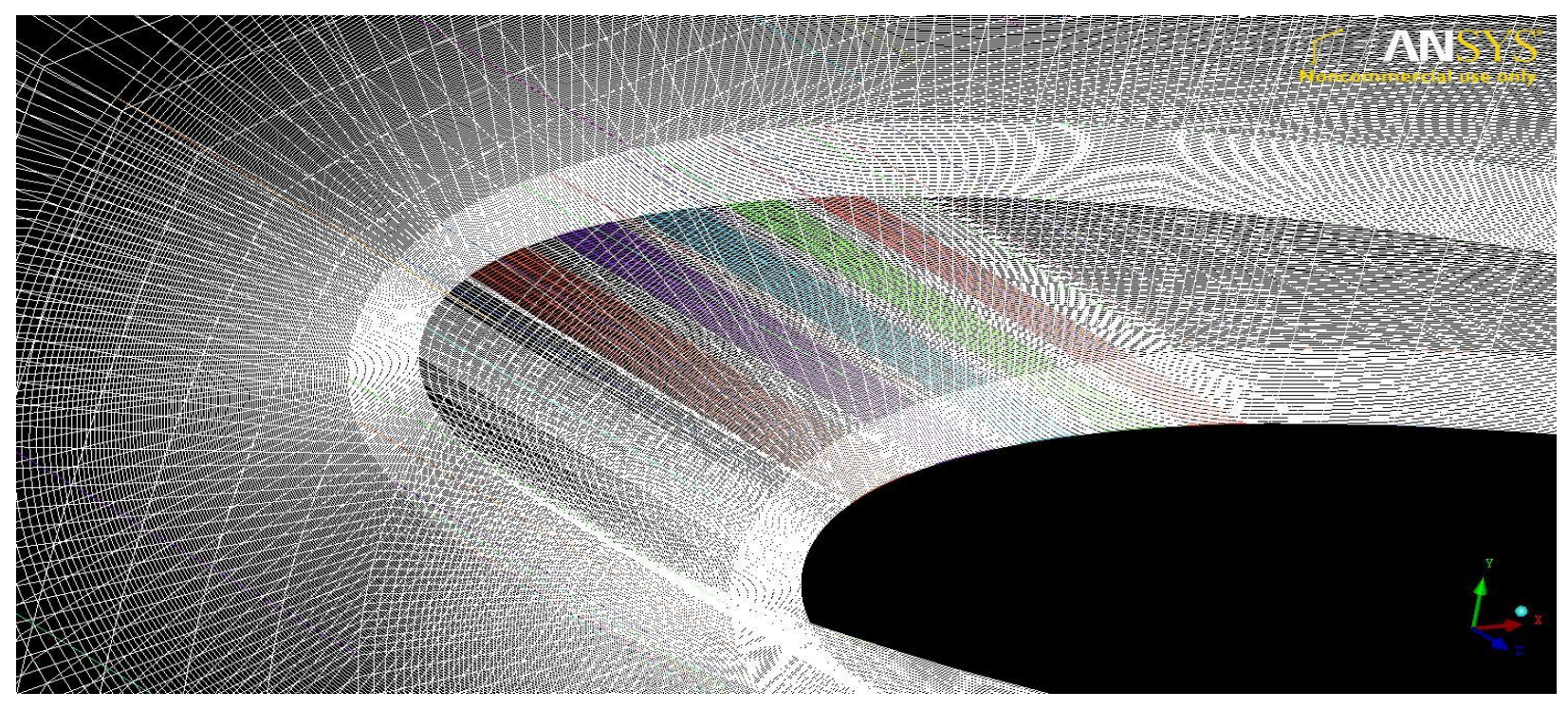

Figure 3.5 3D grid view with ridges

Figure 3-6 shows the locally enlarged view with focus on the locations of ridges.

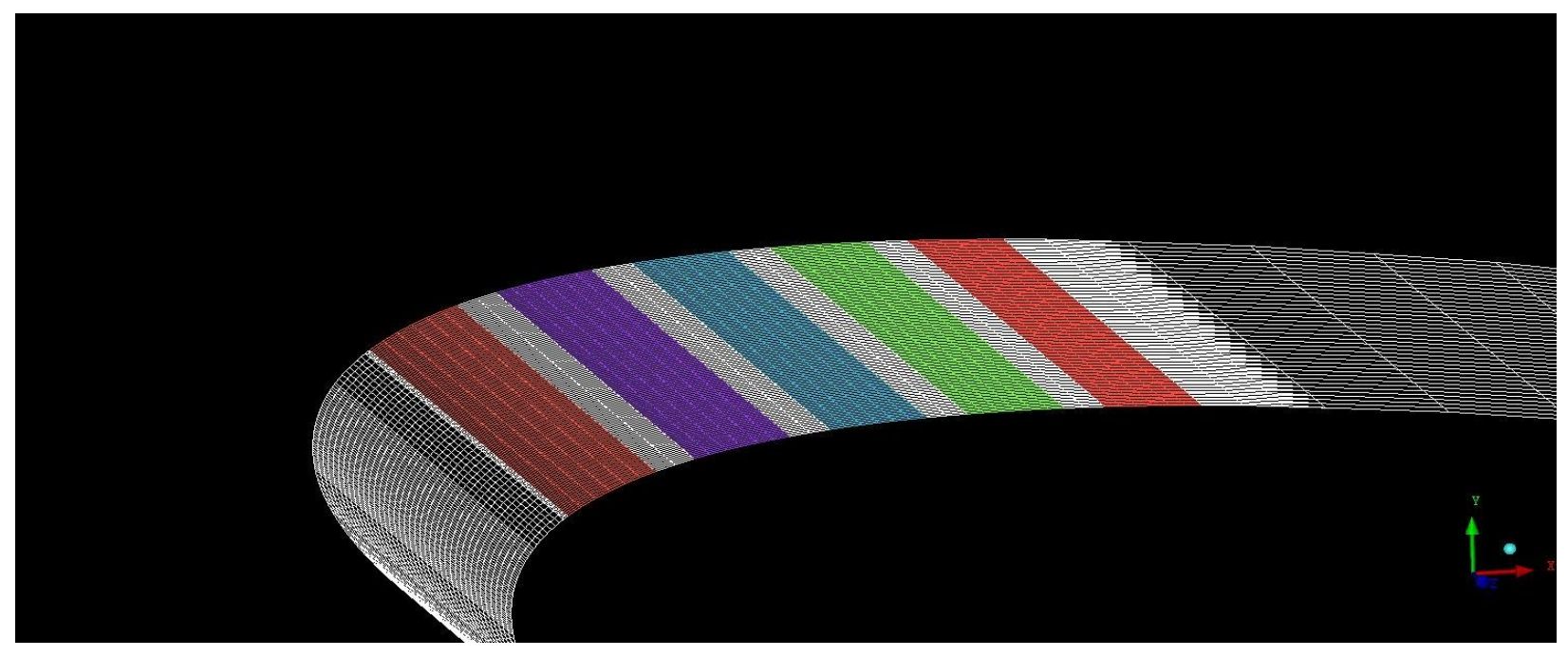

Figure 3.6 Ridges which transform into humps 


\subsection{Hump Generation}

A universal UDF which transforms ridges to desired hump pattern has been developed. One can specify the required hump size, the spacing between humps and required amplitude to get the desired hump pattern. This is a new technique of hump generation. Earlier studies (6) and (8) have been done on meshes with individual humps. Meshing had to be done separately on each hump with blocking being done on each of those humps. This consumes a lot of time due to the complexity involved in meshing these humps. Figure 3-7 shows the complex blocking involved in generating a mesh with individual humps.

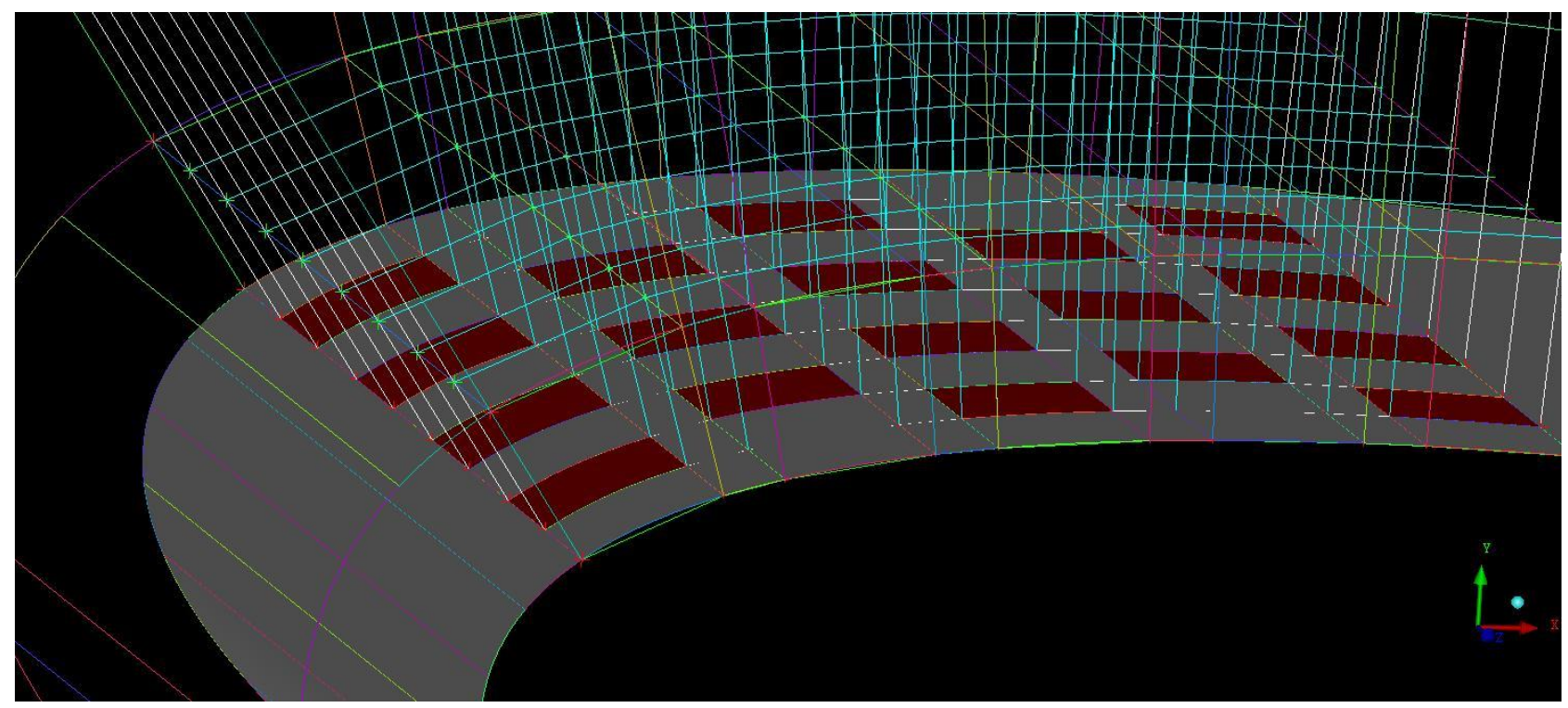

Figure 3.7 Complex blocking with individual humps

On the other hand, the blocking of ridges is very easy and there is hardly any complexity involved with this as done in this study. It is less time consuming in terms of hours compared to working on individual humps. Figure 3-8 shows the simple blocking involved with ridges. 


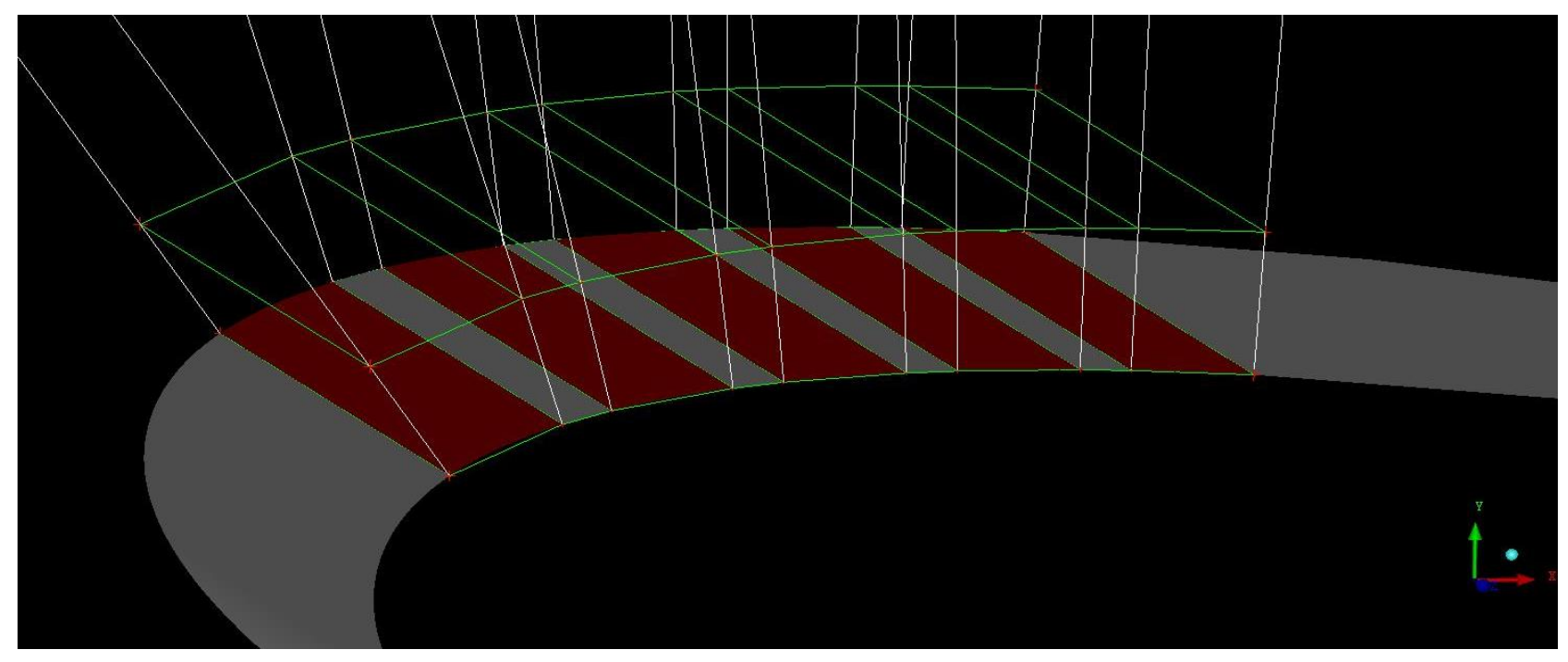

Figure 3.8 Simple blocking with ridges

Keeping this in view, a UDF has been developed which can transform the ridges to the desired hump pattern. The humps operate with the specified frequency and amplitude. One needs to specify the ridge location and hump location. Compared to the earlier specified example where 50 humps were required, this can be easily done with multiple ridges. Figures 3-9 - 3-11 show the humps at various levels of displacement amplitude.

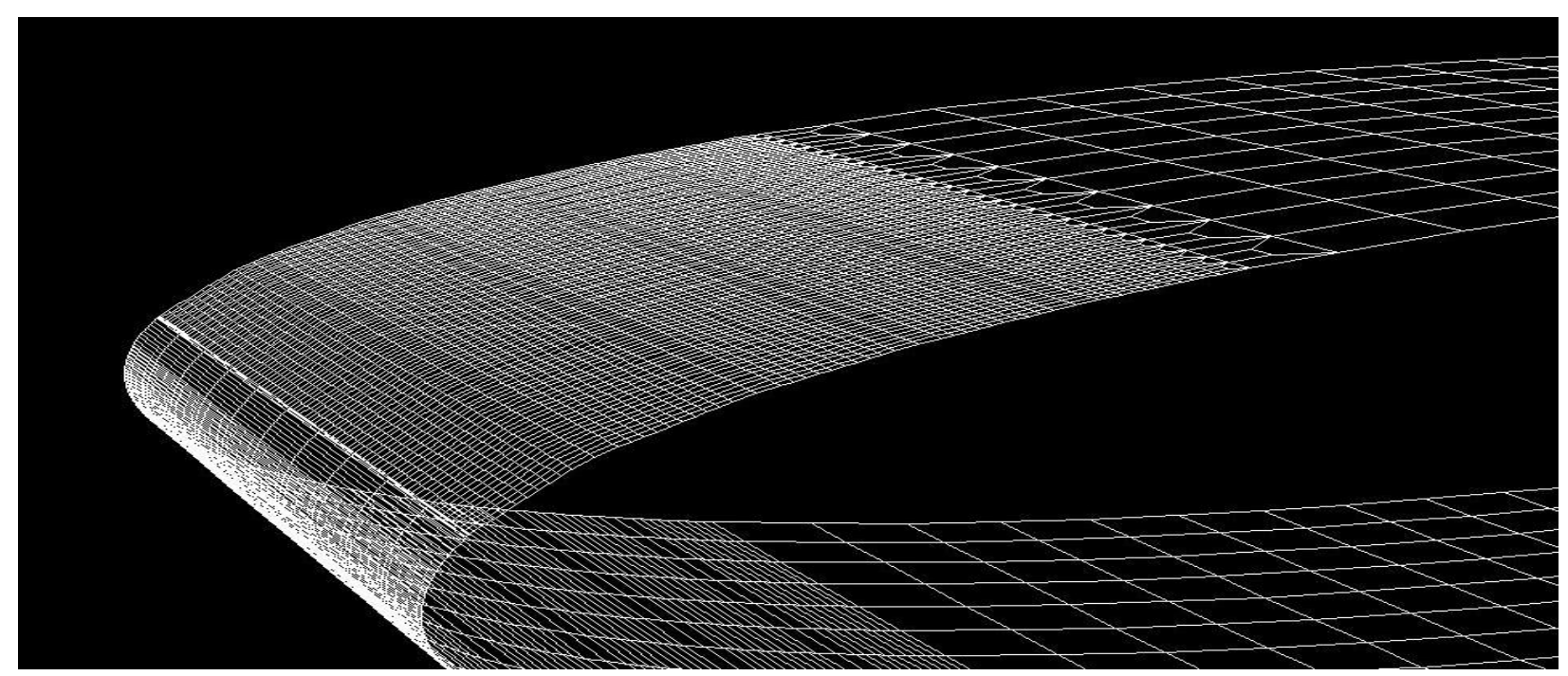

Figure 3.9 Humps at rest 


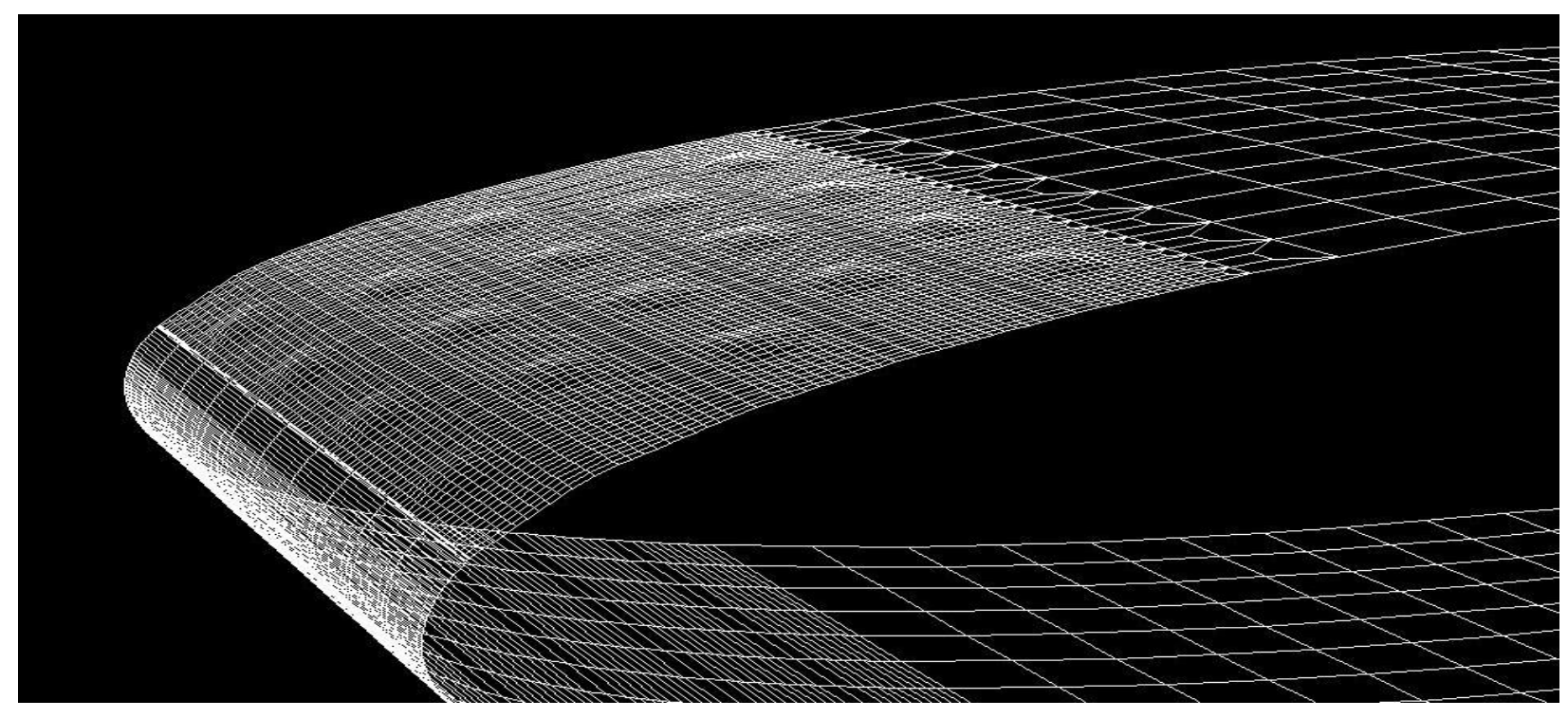

Figure 3.10 Humps at intermediate amplitude

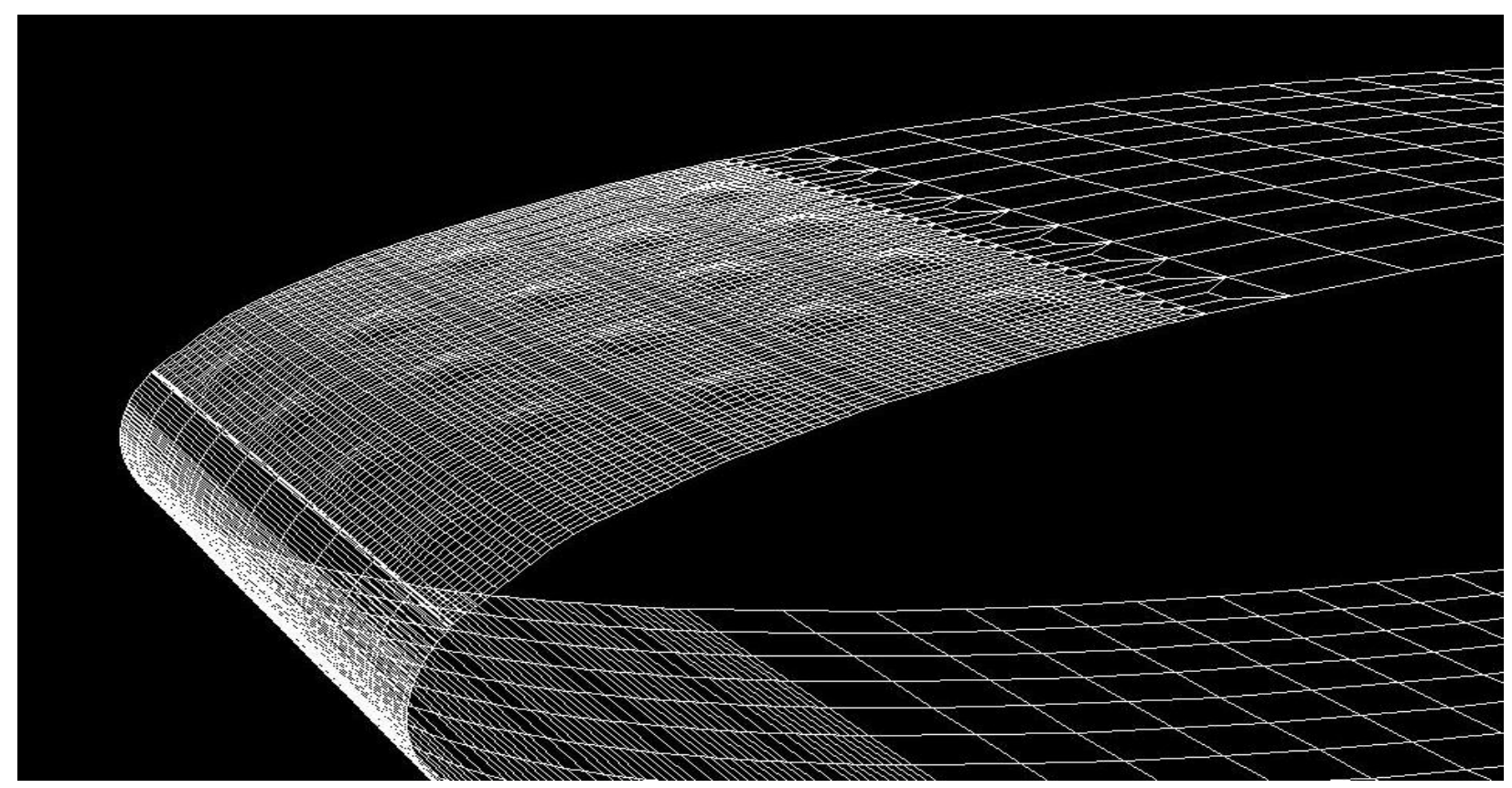

Figure 3.11 Humps at maximum amplitude

The dynamic roughness axially extends from $0.5 \%$ of the chord length to $9.5 \%$. The humps had a diameter of $0.00212 \mathrm{~m}$ and the maximum amplitude is $0.00023 \mathrm{~m}$, while the airfoil chord is $0.15 \mathrm{~m}$ The humps operate at a frequency of $90 \mathrm{~Hz}$. This frequency was used by Grager (10) in 
all his studies at different Reynolds numbers. From his frequency study, this was the highest frequency at which he observed complete control. He concluded that flow control increases with increase in amplitude (but still within boundary layer height) and also with an increase in frequency of operation. The span wise spacing between each hump is $0.00226 \mathrm{~m}$. The humps were in a staggered configuration with four humps in the first, third and fifth rows and three humps in the second and fourth row.

There has to be a certain number of grid points on each hump for it to be a proper hump without any deformations. It has been found that the UDF generates smooth and proper humps if there are at least seven grid points passing over each hump area in both the directions. For lesser grid points, the UDF generated faceted humps. Hence considering this, each hump has 13 grid lines in the span wise direction and 7 in the stream wise direction.

Figure 3-12 shows the blocking over the dynamic roughness region. The blocks in violet represent the deforming zone. Dynamic meshing i.e. the mesh movement is done with the smoothing technique. The edges between any two mesh nodes are idealized as a network of interconnected springs. The initial spacing of the edges before any motion constitutes the equilibrium state of the mesh. A displacement at a given boundary node will result in displacement along all springs connected to the node. In this process, the nodes are smoothly displaced and come back to original position when the hump moves up and down Gall (8). 


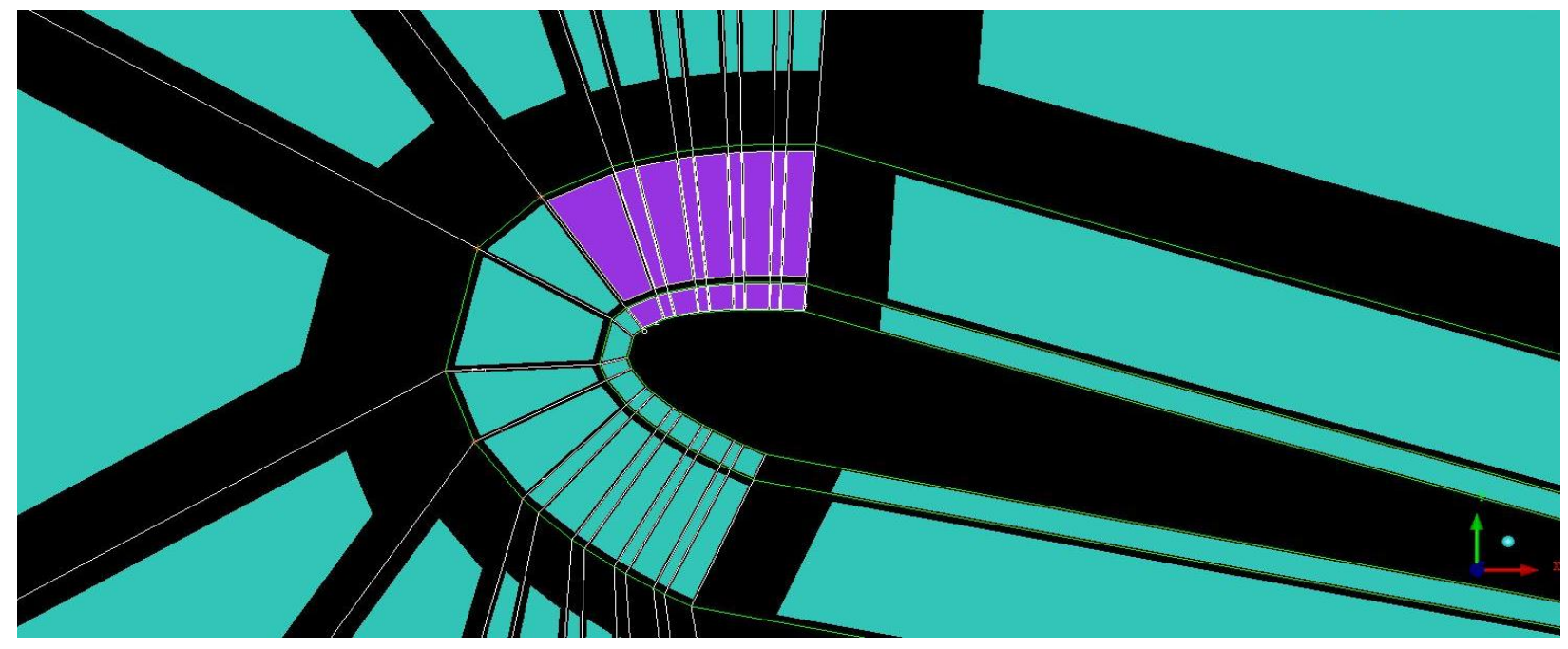

Figure 3.12 Deforming zone in the mesh

A UDF has been developed which transforms the ridges to the staggered humps pattern. This UDF is listed in the appendix. The amplitude of the humps is 230 microns and the frequency is $90 \mathrm{~Hz}$. This has been implemented by the dynamic mesh method in FLUENT. Table 3-1 gives the dynamic meshing parameters.

Table 3-1 Dynamic mesh parameters

\begin{tabular}{|c|c|}
\hline Dynamic mesh method & Smoothing \\
\hline Spring constant factor & 0 \\
\hline Convergence tolerance & $0.001 \mathrm{~m}$ \\
\hline Minimum Length Scale & $0.0003 \mathrm{~m}$ \\
\hline Maximum Length Scale & $0.0009 \mathrm{~m}$ \\
\hline Maximum Skewness & 0 \\
\hline
\end{tabular}

In order to have at least 10 elements going over each hump in $\mathrm{Z}$ direction with four humps in a row and with five gaps in between, a total of above 90 nodes have to be defined in the $\mathrm{Z}$ 
direction. This would result in a very large mesh and the computational resources will not be enough to perform the numerical study. So using a method in ICEM, the number of nodes has been specified such that there are 117 nodes over the DR region and only 39 elements at all other areas in $\mathrm{Z}$ direction. This is clearly visible in Figures 3-11 and 3-12. The mesh has a size of 3.3 million cells. The mesh basically has a top wall, a bottom wall, front and back planes, inlet and outlet and the airfoil with ridges. The ridges transform into humps in a staggered pattern on application of the UDF. Figures 3-13 and 3-14 show the nature of the nodes when the hump is at rest and when it is expanding.

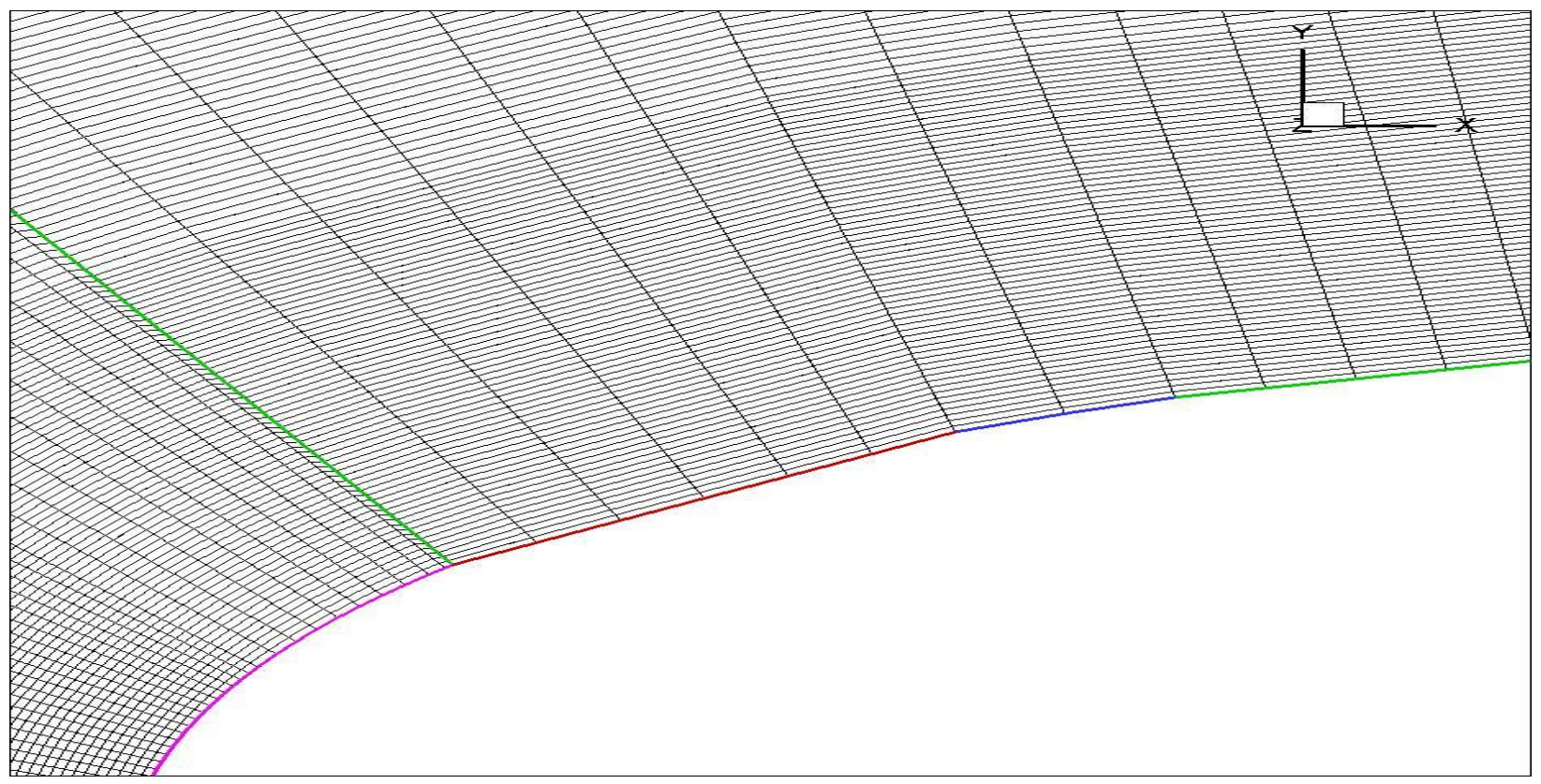

Figure 3.13 Hump at rest 


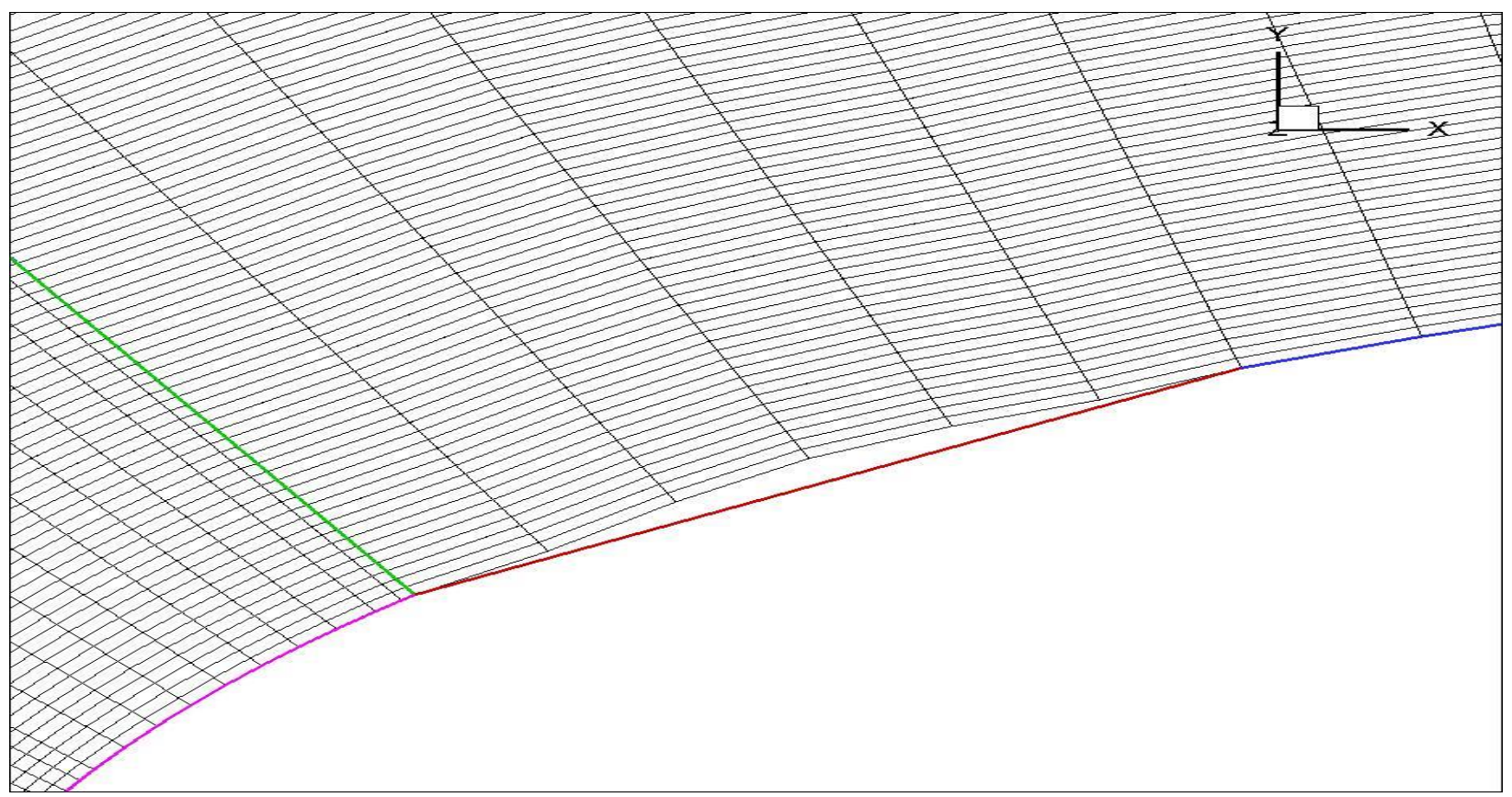

Figure 3.14 Hump in motion

\subsection{Ridge to Row of Humps transformation}

Earlier each hump had its geometry defined and all specific properties of its motion written exclusively for itself in the UDF. The same concept has been extended to an entire surface stretching out in the span wise direction. If the entire surface moves up and down, (similar to a speed bump) it is known as a ridge. This ridge is basically a surface that displaces up and down. In this study, instead of transforming a surface into a ridge, the geometry equations and functions have been modified such that the entire surface instead of transforming into a ridge could transform into staggered rows of humps. So certain portions of the surface were left idle and only certain portions would involve in motion. This was done by basically modifying the functions in the UDF that govern the shape of the hump. This saves a lot of time by eliminating the process of meshing each hump separately. 


\subsection{FLUENT Parameters for LES}

The generated mesh has been imported into FLUENT and for this study the Kinetic-Energy Transport LES model has been used. The current study has been performed at a Reynolds number of 49000 . The inlet velocity for this case is $5.3 \mathrm{~m} / \mathrm{s}$ and the airfoil chord is $0.15 \mathrm{~m}$. Time step size that has been used for this study is $3.2 \mathrm{e}-06 \mathrm{~s}$. The number of iterations per time step is 9000. Periodic boundary conditions have been used for the front and back planes (side planes). Also they are translationally periodic. These are used when the flows across the two opposite planes are identical. Translationally periodic boundaries are boundaries that from periodic planes in a rectilinear geometry. Table 3-2 gives the properties of air used in this study. Table 3-3 lists solver parameters.

Table 3-2 Properties of air

\begin{tabular}{|c|c|}
\hline Property & Value \\
\hline Density & $1.165\left(\mathrm{~kg} / \mathrm{m}^{3}\right)$ \\
\hline Viscosity & $1.8873 \mathrm{e}-05(\mathrm{~kg} / \mathrm{m}-\mathrm{s})$ \\
\hline
\end{tabular}

Table 3-3 gives the Solver parameters used in this study 
Table 3-3 Solver parameters

\begin{tabular}{|c|c|}
\hline Viscous Model & LES \\
\hline Solver & Pressure-Based \\
\hline Reference area & $0.002925\left(\mathrm{~m}^{2}\right)$ \\
\hline Pressure Velocity Coupling & PISO \\
\hline Spatial Discretization & \\
\hline Gradient & Green-Gauss Cell based \\
\hline Pressure & PRESTO \\
\hline Momentum & Bounded Central Differencing \\
\hline Subgrid Kinetic Energy & Bounded Central Differencing \\
\hline Transient Formulation & $\begin{array}{c}\text { Second order implicit (Clean) } \\
\text { First order implicit (DR) }\end{array}$ \\
\hline Residuals Convergence Criteria & 0.001 \\
\hline
\end{tabular}

The PISO algorithm is generally highly recommended for all transient flow calculations. PRESTO discretization gives more accurate results than Standard since interpolation errors and pressure gradient assumptions on the boundaries are avoided. Central differencing scheme is an ideal choice for LES because of its low numerical diffusion. However it leads to unphysical oscillations in the solution fields. The situation is generally made worse in LES by usually very low subgrid scale turbulent diffusivity. The bounded central differencing scheme is essentially 
based on the NVD or Normalized Variable Diagram approach together with convection boundedness criterion. Like the TVD scheme, the NVD scheme is also based on an unboundedness indicator which identifies parts of the domain where intervention is necessary in discretization method. It introduces a procedure in which the discretization practice is locally adjusted for the convection term based on local shape of the solution. The bounded central differencing scheme is a composite NVD-scheme that consists of a pure central differencing, a blended scheme of central differencing and second-order upwind scheme, and the first-order upwind scheme. This scheme hence prevents any unnecessary fluctuations in the solution fields. The transient formulation is second order for clean and first order for DR as the dynamic meshing in FLUENT by default allows only first order formulation.

Table 3-4 lists the under-relaxation factors used for the current study. These are the default values in FLUENT.

Table 3-4 Under-relaxation factors

\begin{tabular}{|c|c|}
\hline Pressure & 0.3 \\
\hline Density & 1 \\
\hline Body forces & 1 \\
\hline Momentum & 0.7 \\
\hline Subgrid Kinetic energy & 0.8 \\
\hline Turbulent Viscosity & 1 \\
\hline
\end{tabular}

Table 3-5 lists the boundary conditions in this study. 


\section{Table 3-5 Boundary conditions}

\begin{tabular}{|c|c|}
\hline Airfoil \& Humps & Wall \\
\hline Inlet & Velocity- inlet \\
& Components: X velocity $=5.3 \mathrm{~m} / \mathrm{s}$, \\
& Y velocity $=0 \mathrm{~m} / \mathrm{s}$, \\
& Z velocity $=0 \mathrm{~m} / \mathrm{s}$, \\
& Fluctuating Velocity Algorithm - \\
& No perturbations \\
& Subgrid Kinetic Energy Specification method - \\
& Subgrid- Scale K \\
& Subgrid Kinetic energy $=2 \mathrm{e}-05\left(\mathrm{~m}^{2} / \mathrm{s}^{2}\right)$ \\
\hline Outlet & Pressure-outlet \\
\hline Top wall & Wall ( No slip) \\
\hline Bottom wall & Wall ( No slip) \\
\hline Front plane \& Back plane (Side planes) & Periodic \\
\hline
\end{tabular}

Table 3-6 lists the time scales for different meshes.

Table 3-6 List of time scales

\begin{tabular}{|c|c|}
\hline Mesh based on Re & Time step size (s) \\
\hline 49000 & $3.2 \mathrm{e}-06$ \\
\hline 25000 & $7 \mathrm{e}-06$ \\
\hline
\end{tabular}




\subsection{WVU HPC Cluster}

The current numerical study has been performed using FLUENT version 13.0 installed on the WVU High Performance Cluster - MOUNTAINEER. It has 32 compute nodes. Each node has 12 cores and a total of 48 GB RAM providing 4GB per node average. The operating system is RedHat Enterprise Linux 6.3. Job scheduler is Moab running over Torque/PBS. The ideal situation was to use 12 processors on a single node. Using more processors on different nodes actually increased run time as a result of communication between nodes. Typically one flow through time takes on an average about 9-14 days including delays in getting the job through on the cluster as a result of high demand. 


\section{Results}

Experimental work by Grager (10) using PIV clearly showed leading edge stall without the use of dynamic roughness for an airfoil inclined at an angle of $14^{\circ}$ at a Reynolds number of 49000 . Dynamic roughness has been used to control the flow. In the present work, numerical studies have been performed to first simulate the leading edge stall for the same flow conditions, and results have been compared with experiments. Then a numerical study has been performed to study the effects of dynamic roughness on the separated flow, and results are again compared with experiments. In simulations, basically the clean case has been simulated first. Once the separated flow has been established, the DR was then actuated. The numerical studies have been performed by means of large eddy simulations with the commercial CFD software Ansys FLUENT version 13.0.

\section{a) Clean case}

Firstly a preliminary study has been performed on a grid with 1.6 million cells having only 10 mesh elements $(1.3 \% \mathrm{c}$ each) in the span wise direction. All other parameters are the same as mentioned in the earlier chapters. The time step size used for this study was $3.2 \times 10^{-6} \mathrm{~s}$. One flow through time is about $0.0277 \mathrm{~s}$. The clean case has been run for 20 flow through times. Then it has been time averaged for an additional 10 flow through times. Twelve processors have been used on a single node for these runs. It took about 2 days to compute 1 flow through time. Figure 4-1 shows the comparison of turbulence kinetic energy (TKE) contours for the clean case in this study with TKE contours from experimental PIV data. Figure 4-1a represents experimental work (10) and Figure 4-1b represents the present simulation results. It can be clearly seen that the TKE is not captured accurately. It was found that the grid resolution or fineness was not sufficient in 
the span wise direction. So the grid had to be made finer in the span wise direction and the number of cells was increased to 39 in that direction. Figure 4-2 shows the comparison of velocity magnitude contours for the clean case with experimental PIV for the grid with 39 elements in span wise direction and 3.3 million cells. Figure 4-2a represents experimental work and Figure 4-2b represents the simulation results. Figure 4-3 shows the TKE contour comparison with experiments.

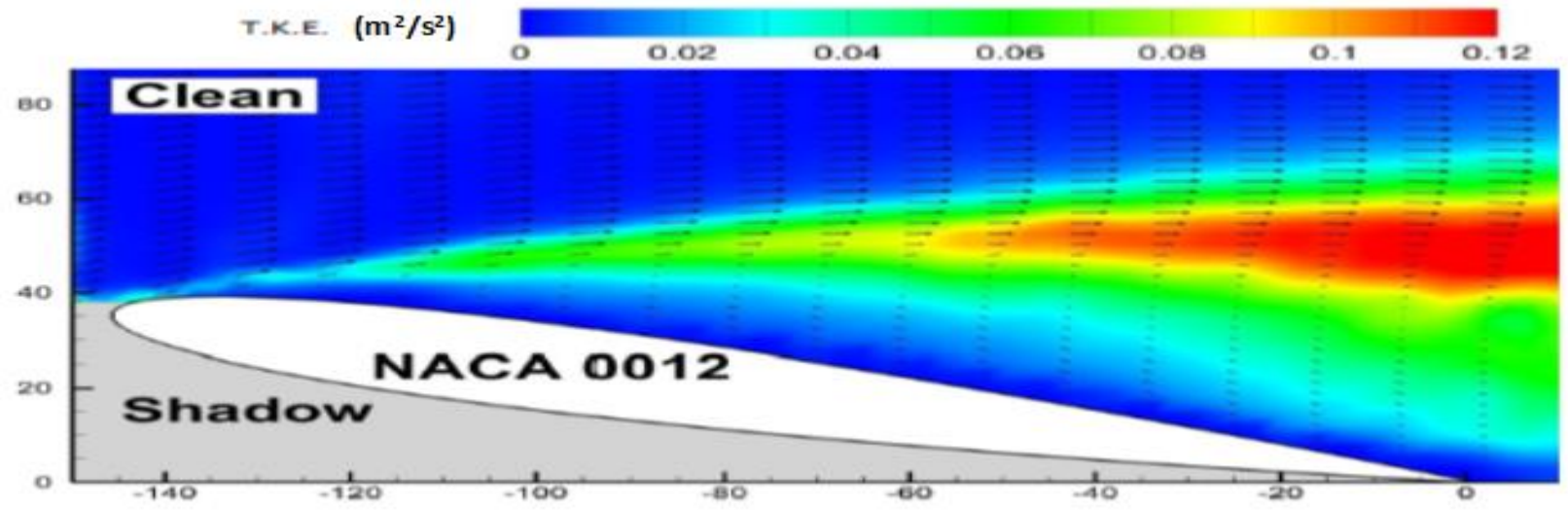

a) TKE contour from experiments (10)

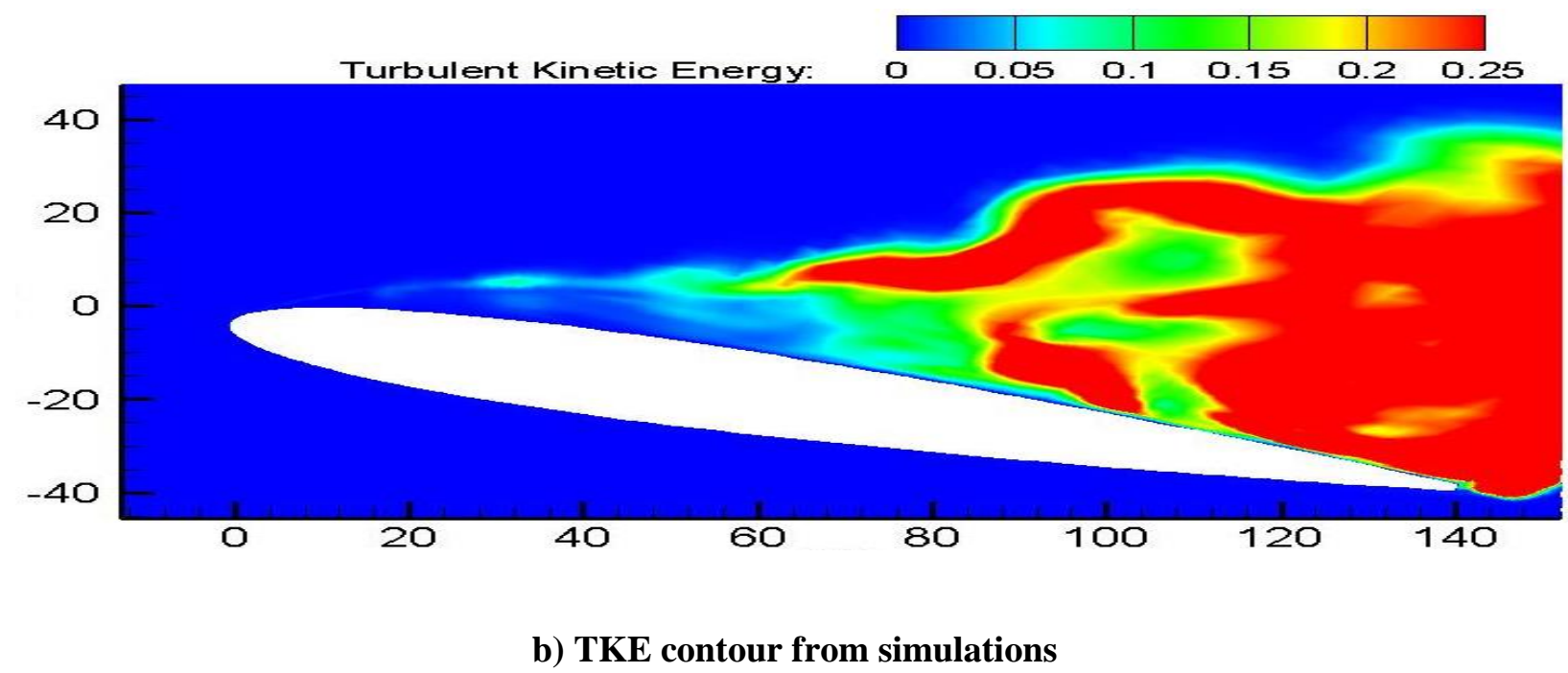

Figure 4.1 Comparison of TKE contours from experiments and simulations (preliminary) for clean case 

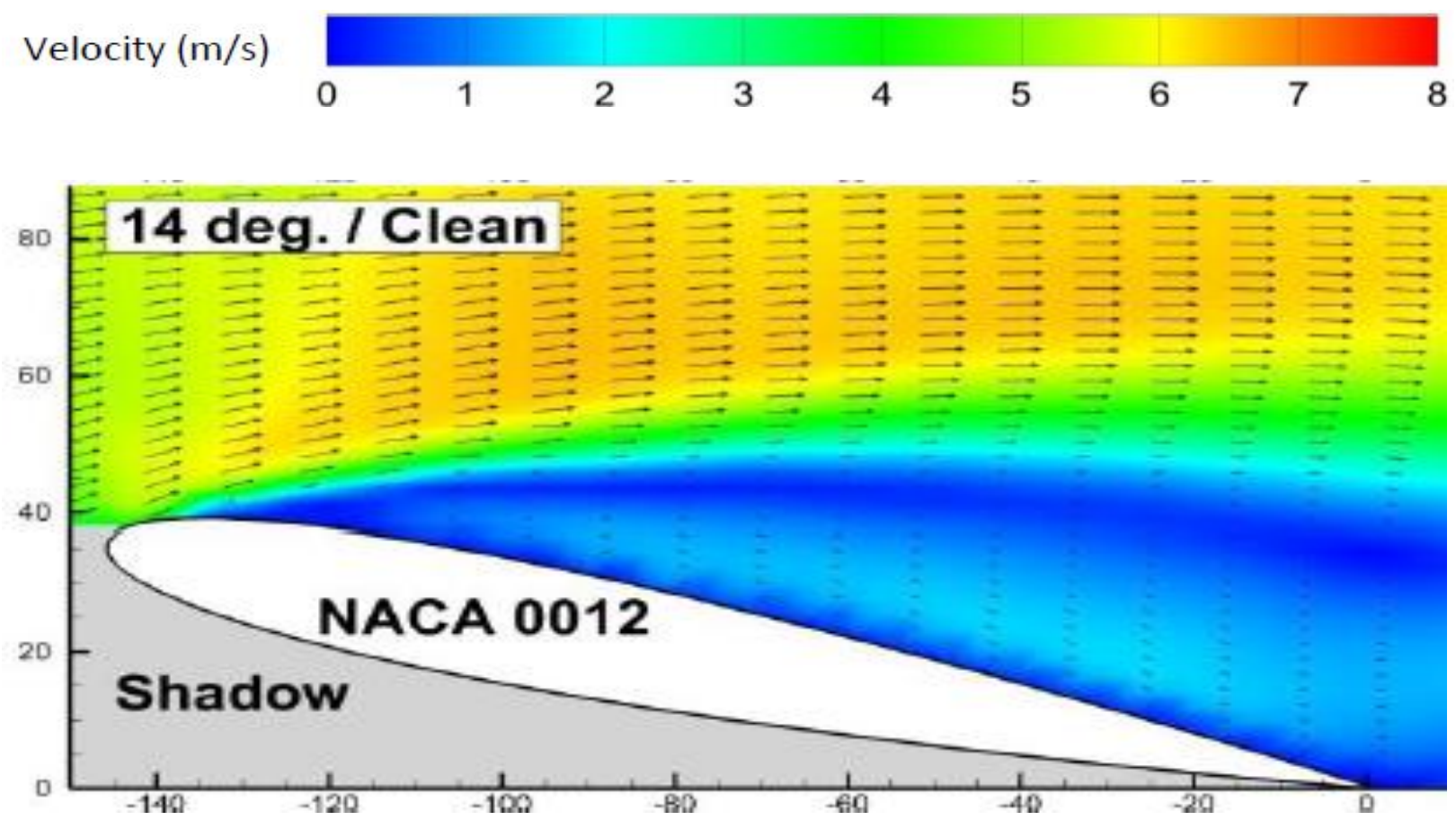

a) Velocity magnitude contour from experiments (10)

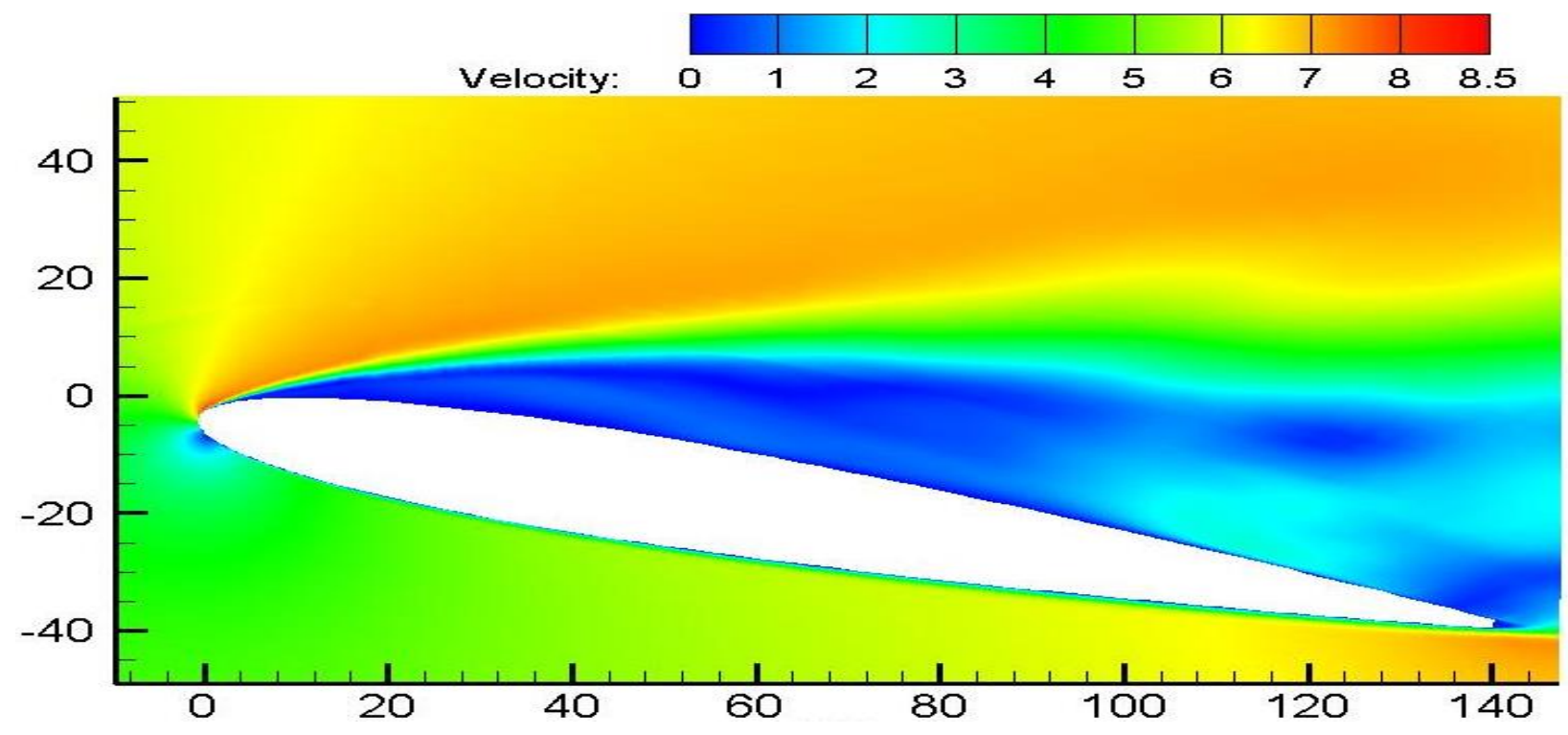

b) Velocity magnitude contour from simulations

Figure 4.2 Comparison of velocity magnitude contours for clean case 


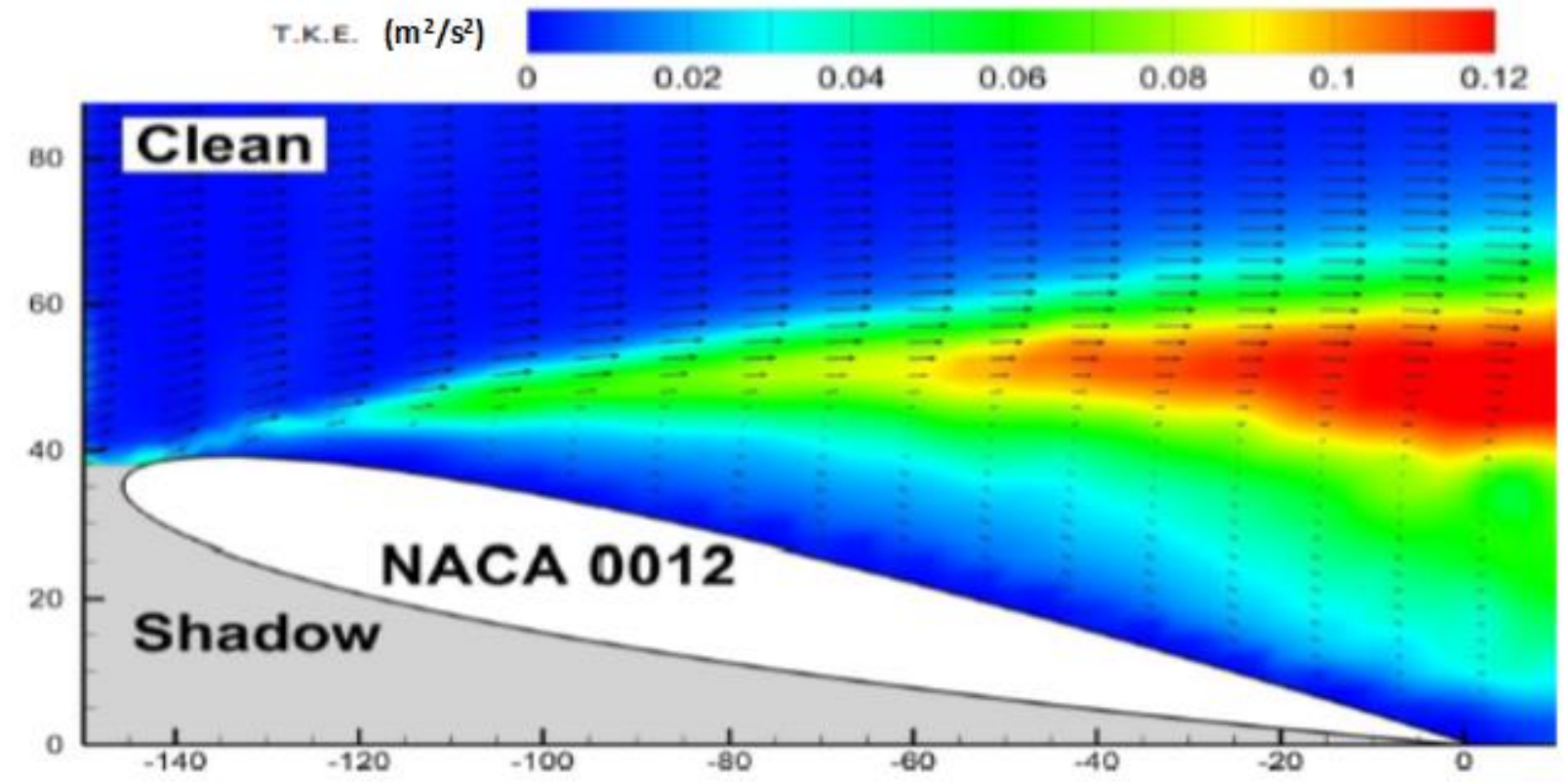

a) TKE contour from experiments (10)

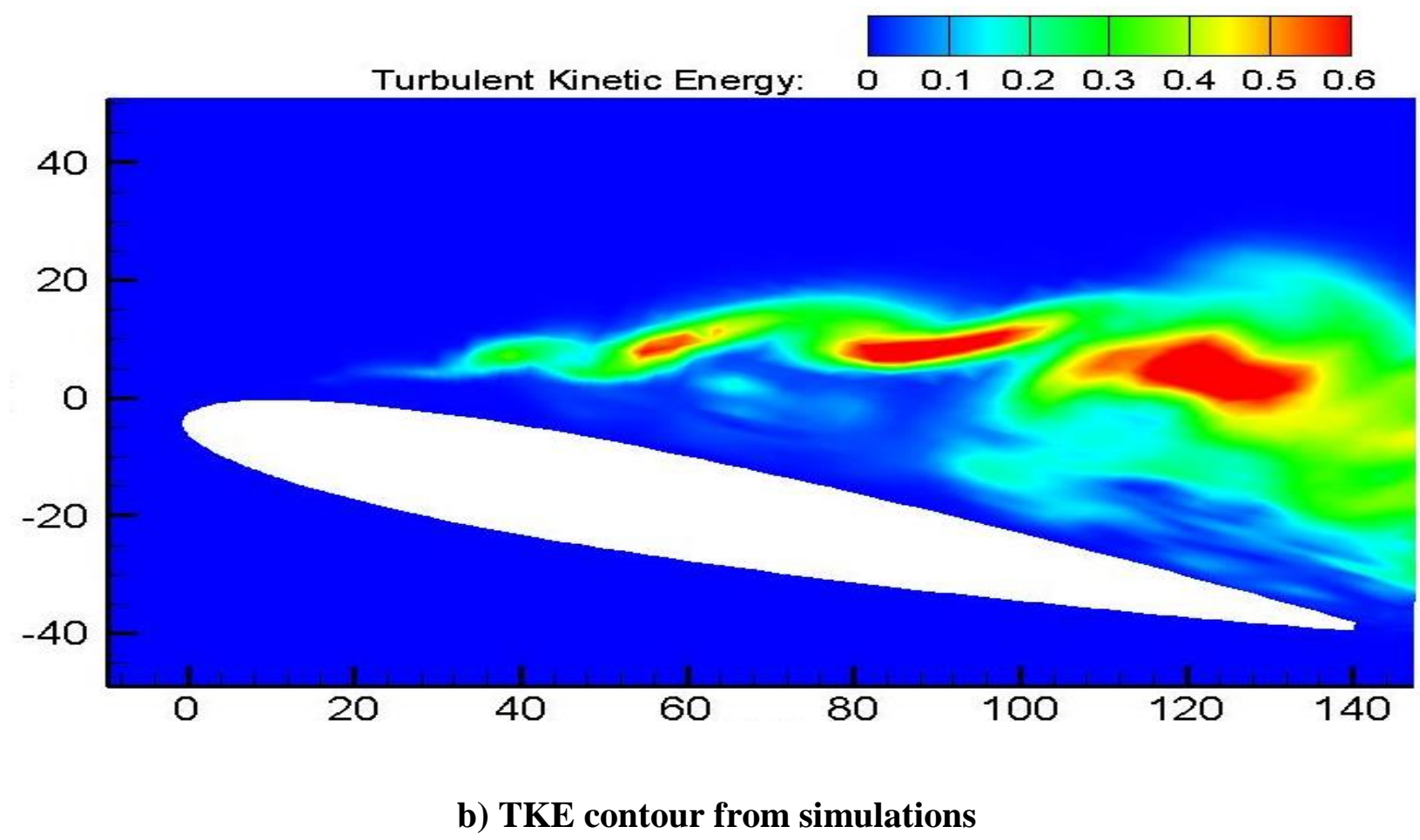

Figure 4.3 Comparison of TKE contours for clean case 
It can be clearly seen from Figure 4-2 that there is quite a close match between the velocity magnitude contours qualitatively from experiments and simulations qualitatively. The flow has separated away from the airfoil. Leading edge stall can be clearly seen from both the experimental and simulation plots. Also, the general shape of the turbulent kinetic energy contours match quite closely. The structure is being captured quite well. No TKE or very low values are observed along the surface of the airfoil as in experiments. However, there still is a difference in the contour level values, and this might be due to the difference in the turbulence levels in the wind tunnel and the simulation. Refining the grid in span wise direction did capture turbulence pretty well qualitatively. Figure 4-4 shows the comparison of vorticity contours for the clean case with experimental PIV. Figure 4-4a represents experimental work and Figure 4-4b represents the simulation results. 


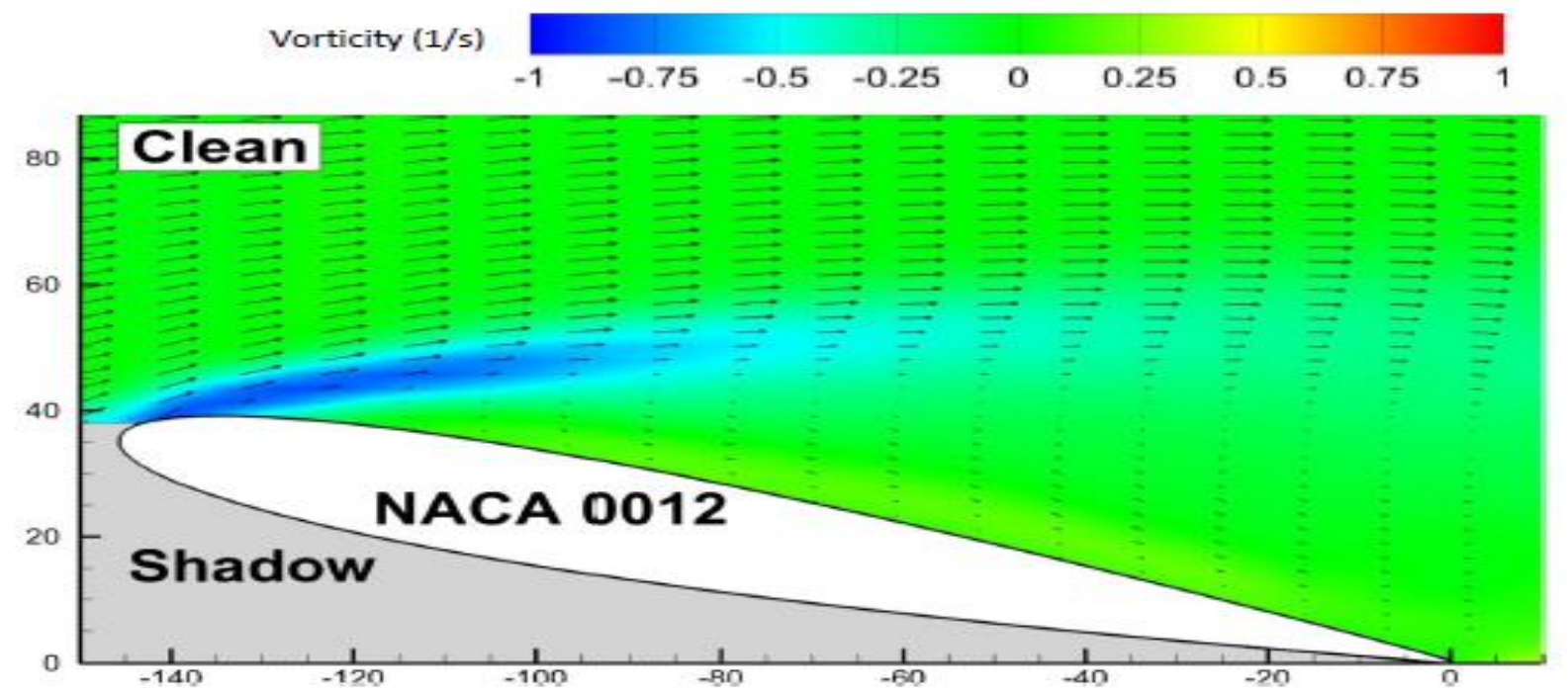

a) Vorticity contour from experiments (10)

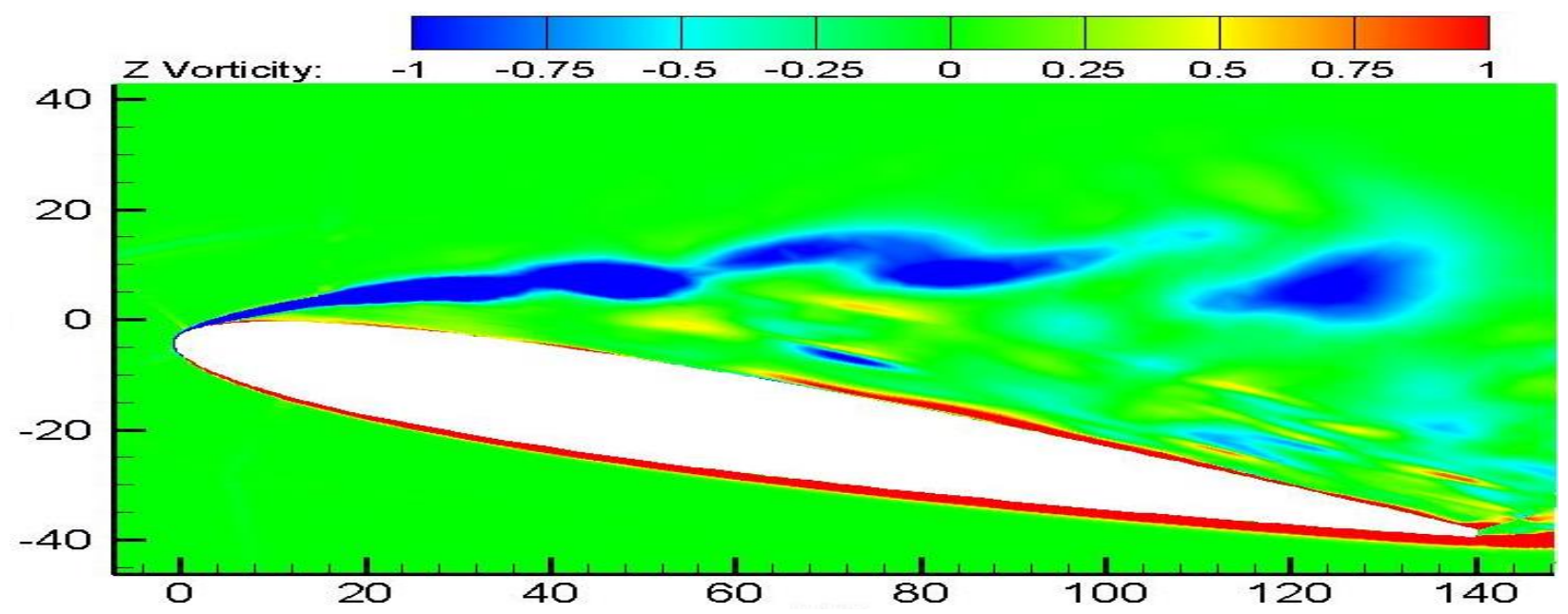

b) Vorticity contour from simulations

\section{Figure 4.4 Comparison of vorticity contours for clean case}

There is a reasonably good match between the vorticity contours from experiments and simulations qualitatively. The trend of the vorticity concentration away from the wall and its separation is being captured pretty well for the clean case. However, the simulation predicts a standing pattern of large scale vortices that either are not present in the experiments or else was 
smeared out by the relatively coarse X-direction resolution in the data. Figure 4-5 shows the velocity contour plot with vectors plotted. Clearly the vectors show flow reversal and separation. The maximum height of the boundary layer has been found to be $0.254 \mathrm{~mm}$ at around $1.3 \% \mathrm{c}$. After that, there is stagnation point and then the flow separates. This value comes into play when the amplitude of the DR is considered.

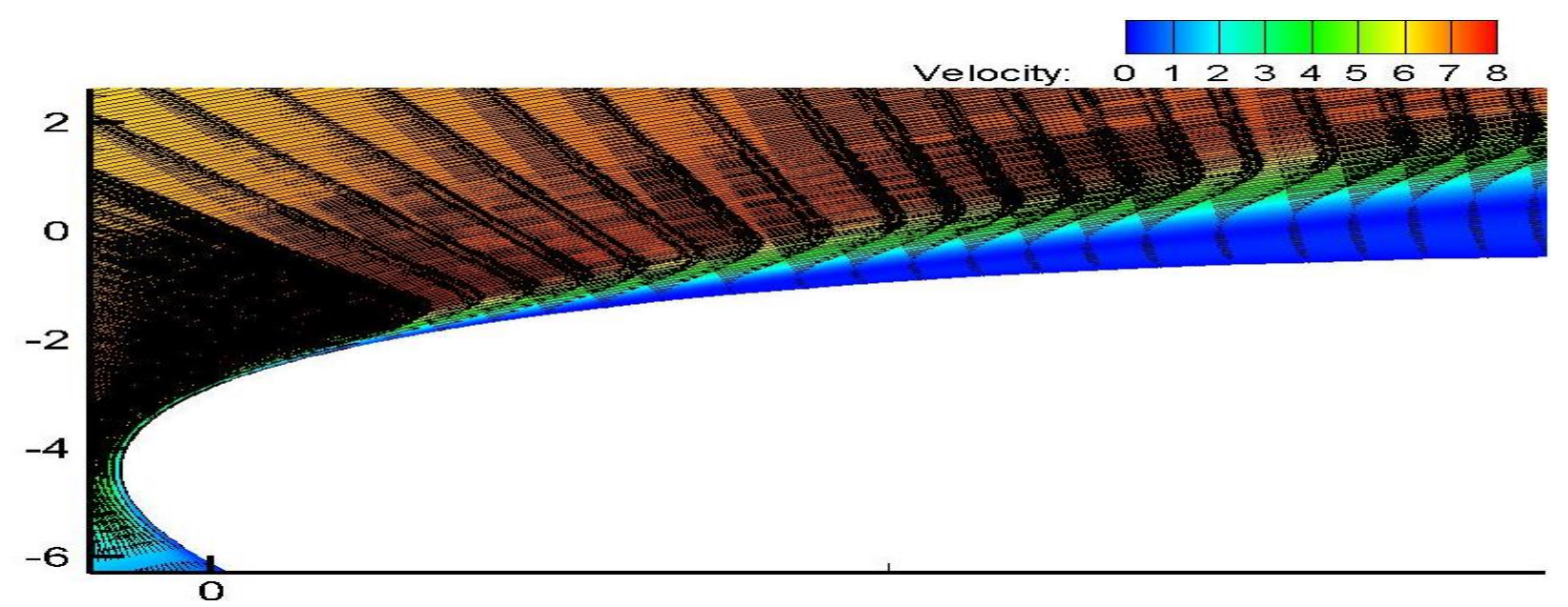

Figure 4.5 Velocity magnitude contour plot with vectors (clean)

\section{b) DR case}

DR has been actuated using the flow field of the clean case. DR case has run for ten flow through times. The results are then time averaged over four flow through times. Figure 4-6 shows the comparison of velocity magnitude contours for the DR case with experiments for the same grid. The DR has a frequency of $90 \mathrm{~Hz}$ and amplitude of 230 microns similar to the experiments. A slight acceleration has been observed due to inclined nature of the grid compared to experimental PIV contours. So in order to make good qualitative comparison between experiments and simulations, different color bars have been used in clean as well as DR plots. Same contour bars show slightly darker region which obscures the visible accelerated region due to DR right over the humps. 

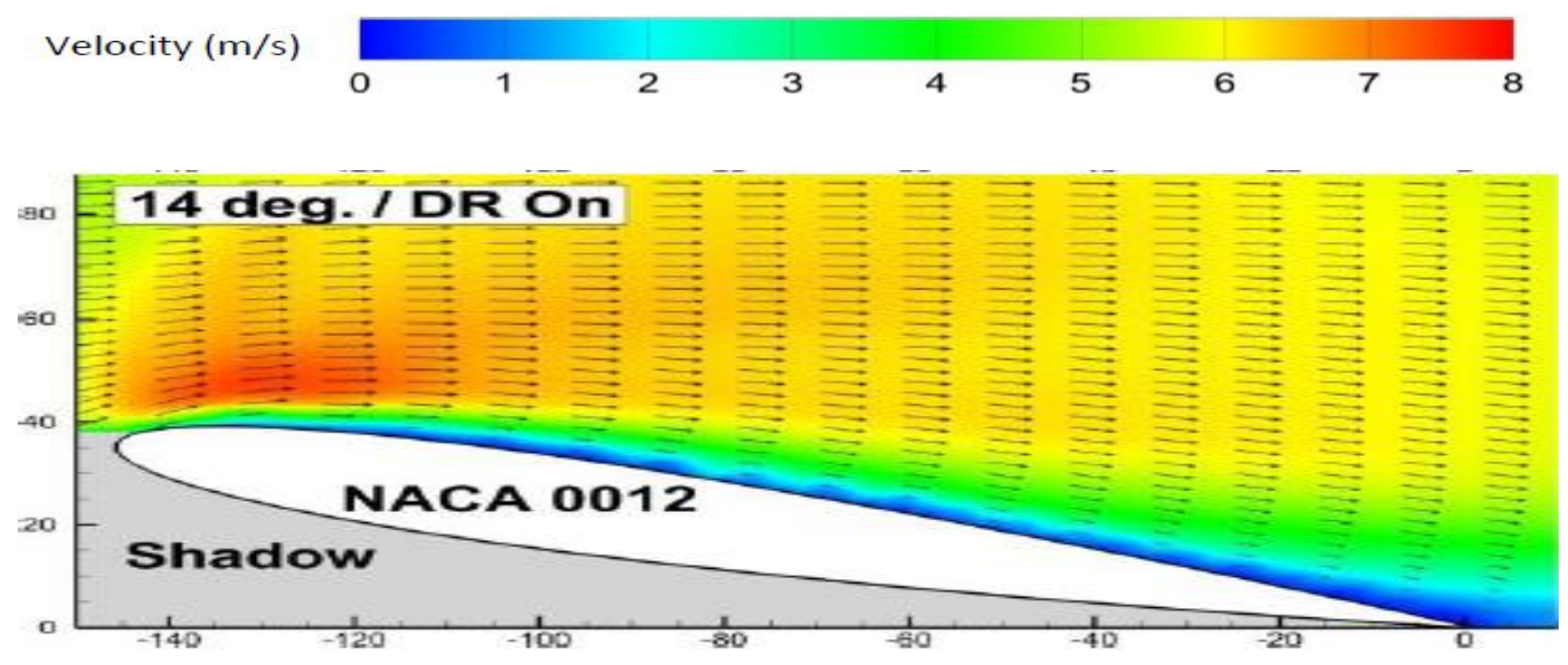

a) Velocity magnitude contour from experiments (10)

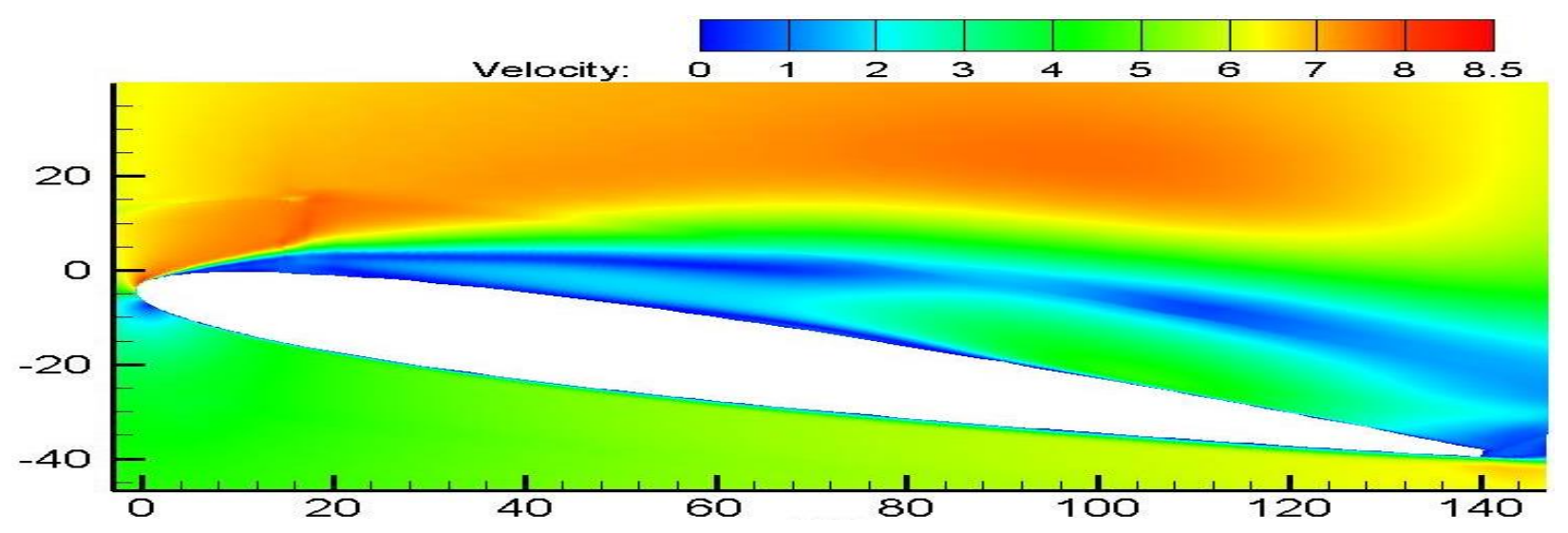

b) Velocity magnitude contour from simulations

Figure 4.6 Comparison of velocity magnitude contours for DR case

Figure 4-7 shows the TKE contour comparison with experiments for the DR case. 


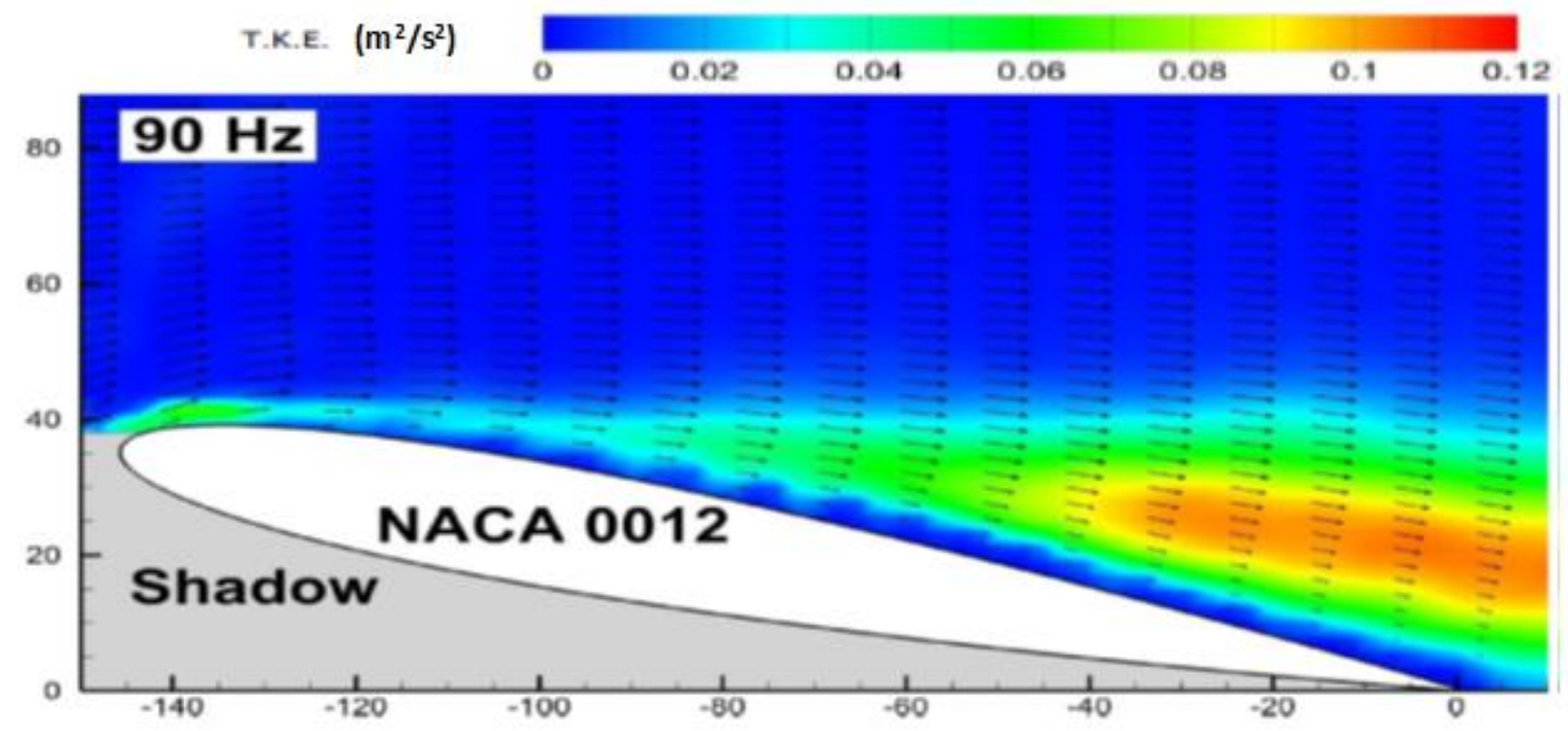

a) TKE contour from experiments (10)

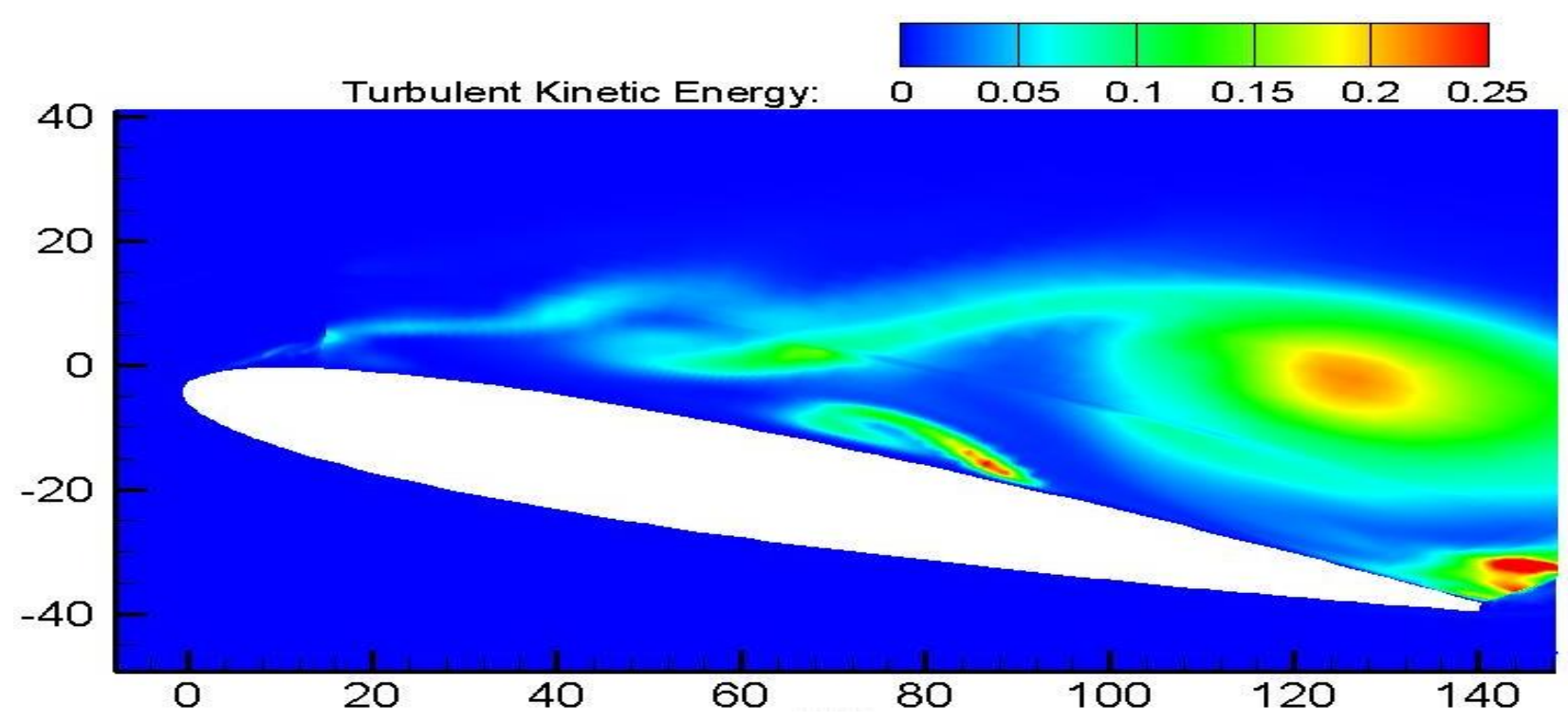

b) TKE contour from simulations

Figure 4.7 Comparison of TKE contours for DR case

Figure 4-6 shows the comparison between the velocity magnitude contours for experiments and simulations. It can be clearly seen from Figure 4-6a that PIV results show full reattachment for this flow condition and DR setting. It can be seen from Figure 4-6 that though there is a slight 
resemblance between the velocity magnitude contours from experiments and simulations for the DR case, the flow is not reattached in the simulation. Similar concentration of higher velocity above the DR region is observed in simulations as found in experiments. DR tries to reattach the flow but there is a huge recirculation region downstream and it continues to have the stall behavior. TKE plots show a somewhat similar structure. However in the simulations, the plot shows slightly lower TKE concentration near the DR region compared to the humps. The plot shows a slight irregularity near the DR region. This might be because TECPLOT post processing software is not able to properly interpolate near the DR region because of differences in the number of cells in the DR region and the regular region beside it. The structure of TKE downstream qualitatively looks somewhat similar to that from experiments, but with higher peak vorticity level located farther from the airfoil surface and a bit upstream. Figure 4-8 represents the comparison between vorticity contours for the DR case for experiments and simulations. Figure 4-8a represents experiments and Figure 4-8b represents simulations. 


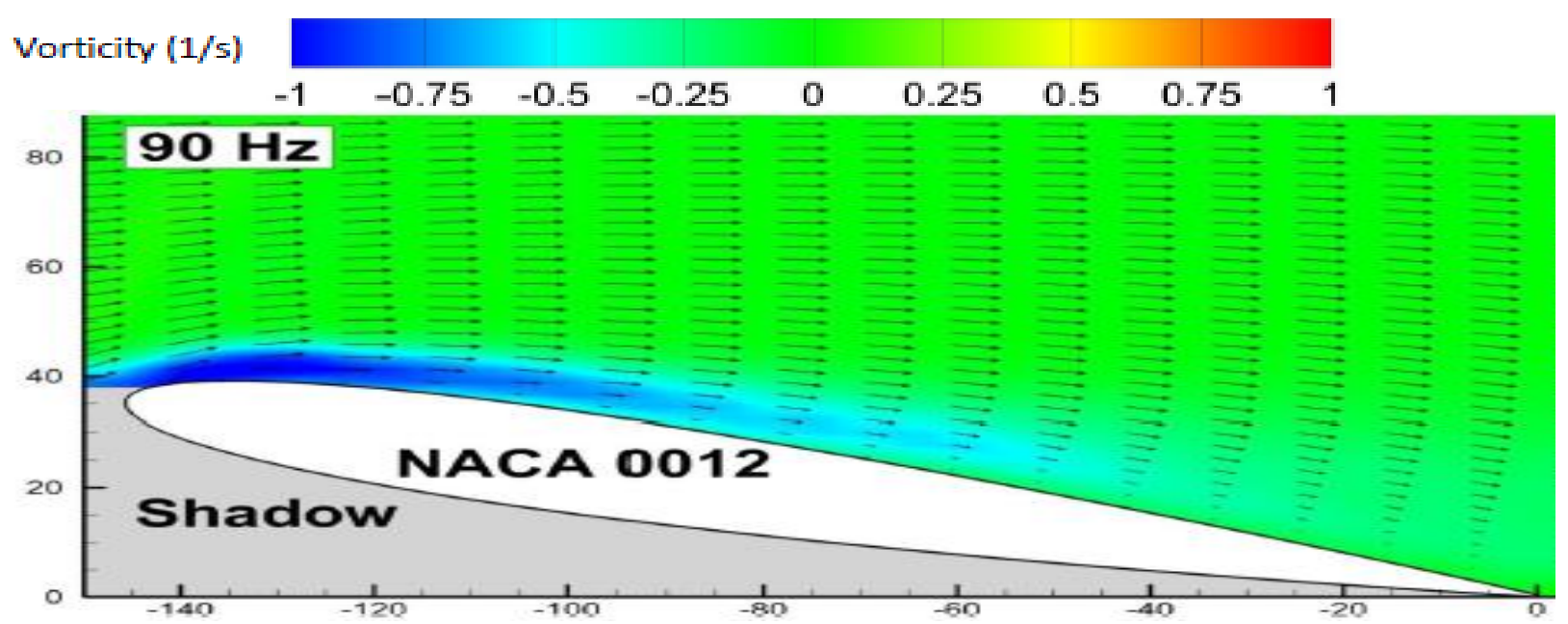

a) Vorticity contour from experiments (10)

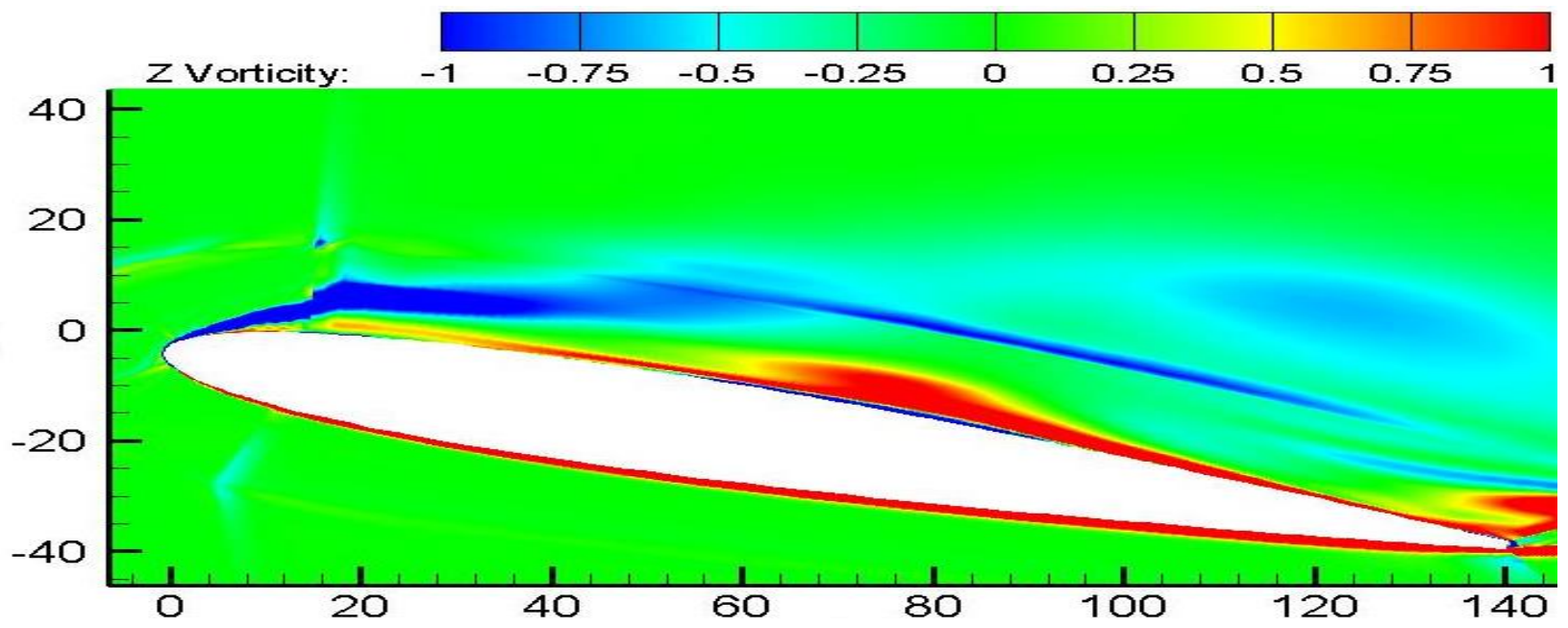

b) Vorticity contour from simulations

Figure 4.8 Comparison of vorticity contours for DR case

There is a reasonable similarity in the vorticity contours from experiments and simulations. There is a higher concentration of vorticity near the DR region indicating the creation of vortices and this results in mixing and entrainment of higher momentum fluid. In comparison to experiments where there is complete flow control, there can be several reasons behind the failure of DR to control the flow numerically. It is likely possible that a much finer grid is necessary to 
achieve flow control. One more possible reason from the CFD side is that $2^{\text {nd }}$ order transient formulation is necessary for the DR case. However FLUENT does not allow that for dynamic meshing. There can be several reasons in experiments which attribute to flow control which do not find a place in simulations. There can be an extra bit of help for flow control due to possible vibrations while actuating DR in experiments. Another possibility is the flow itself being dirty (free stream turbulence being higher) which would have resulted in easier control. Figure 4-9 shows the locally enlarged view of TKE contour for DR case.

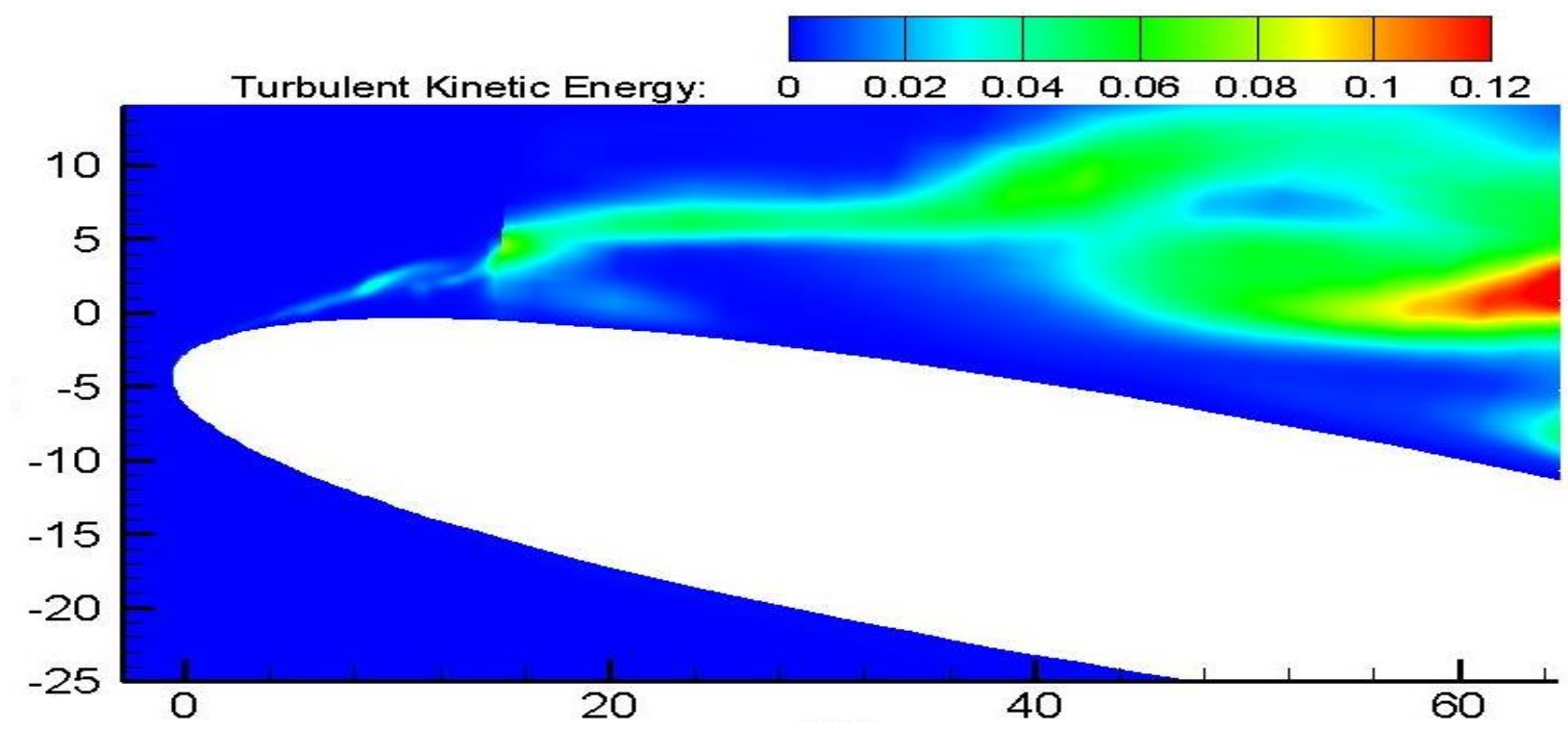

Figure 4.9 TKE contour for DR case (locally enlarged)

Clearly, the locally enlarged picture shows the TKE activity at lower contour levels. This shows that DR is indeed trying to energize the flow. 


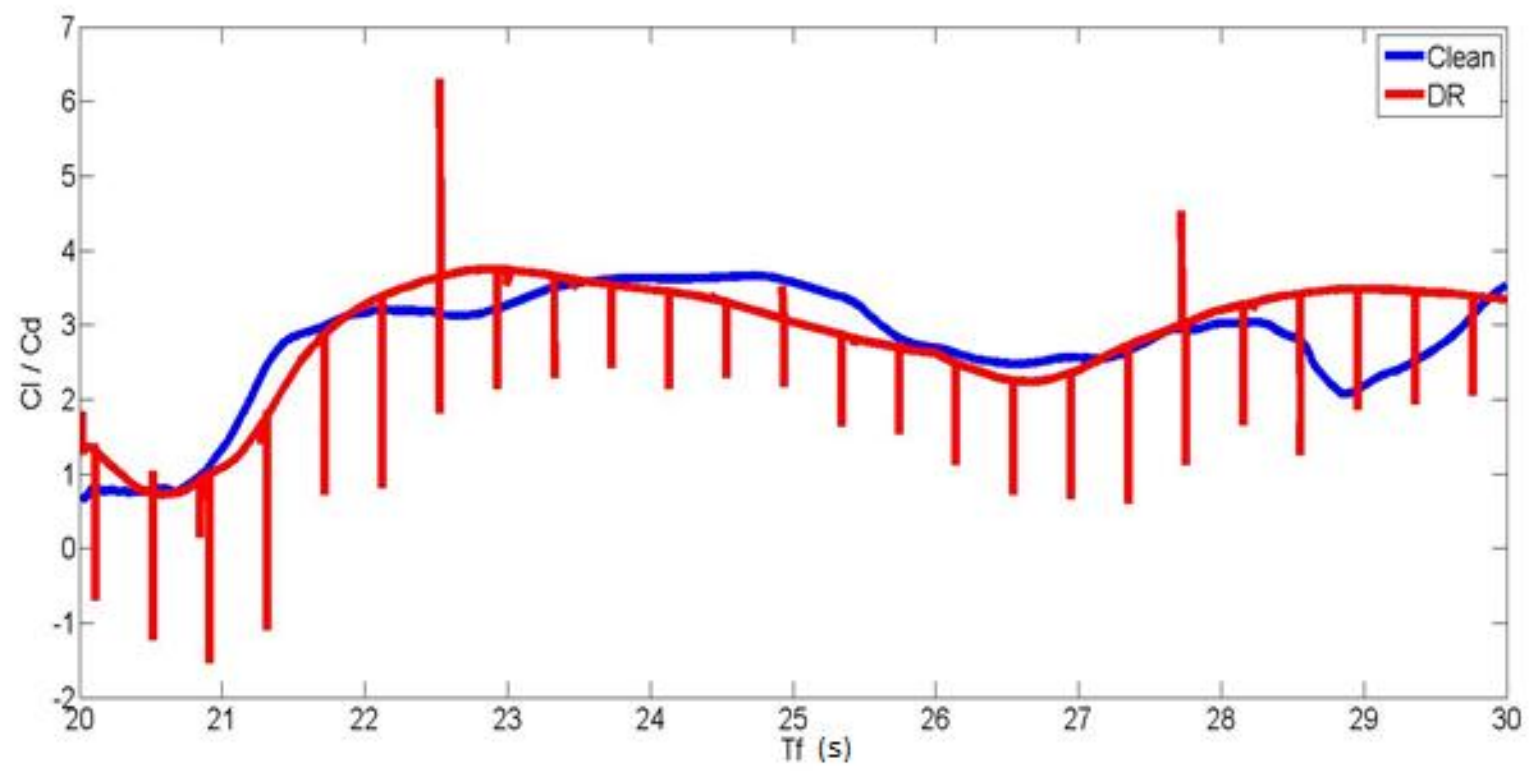

Figure 4.10 Cl / Cd data from simulations

The lift-to-drag drag data plotted as a function of flow-through-time has been presented in the Figure 4-10. The clean case has been represented by the blue line and the DR case by the red line. The spikes are the points in time where humps expand and contract. The mean values of $\mathrm{Cl} / \mathrm{Cd}$ for the clean and DR cases for the simulation are 2.7711 and 2.8460 , respectively. $\mathrm{Cl} / \mathrm{Cd}$ value has increased by $2.7 \%$ in the DR case. The increase would have been much more had total control been achieved. This data has not been collected in the experiments. This inference needs to be validated with experimental data in order to quantify the above effect with more confidence. Table 4-1 lists the height of the separated layer at various chord locations. 
Table 4-1 Height of separated layer (from simulation)

\begin{tabular}{|c|c|c|}
\hline Location $(\% \mathrm{c})$ & Clean $(\mathrm{mm})$ & DR $(\mathrm{mm})$ \\
\hline 13.3 & 4.16208 & 2.72843 \\
\hline 20 & 7.22856 & 5.78314 \\
\hline 26.6 & 9.40874 & 7.76007 \\
\hline 33.3 & 10.8727 & 9.8888 \\
\hline 40 & 12.3349 & 10.8665 \\
\hline 46.66 & 16.4359 & 14.6854 \\
\hline
\end{tabular}

It can be clearly seen that the DR reduces the height of the separated layer. Figure 4-11 shows the mean wall shear stress over the DR region. The clean case clearly shows separation at $0.002 \mathrm{~m}$ i.e. $1.3 \% \mathrm{c}$. There is stall clearly. DR tries to attach the flow, however separation still continued. The peaks and dips show attachment and detachment. Figure 4-12 shows the mean pressure coefficient over DR region for both clean and DR cases. DR tries to increase the suction pressure.

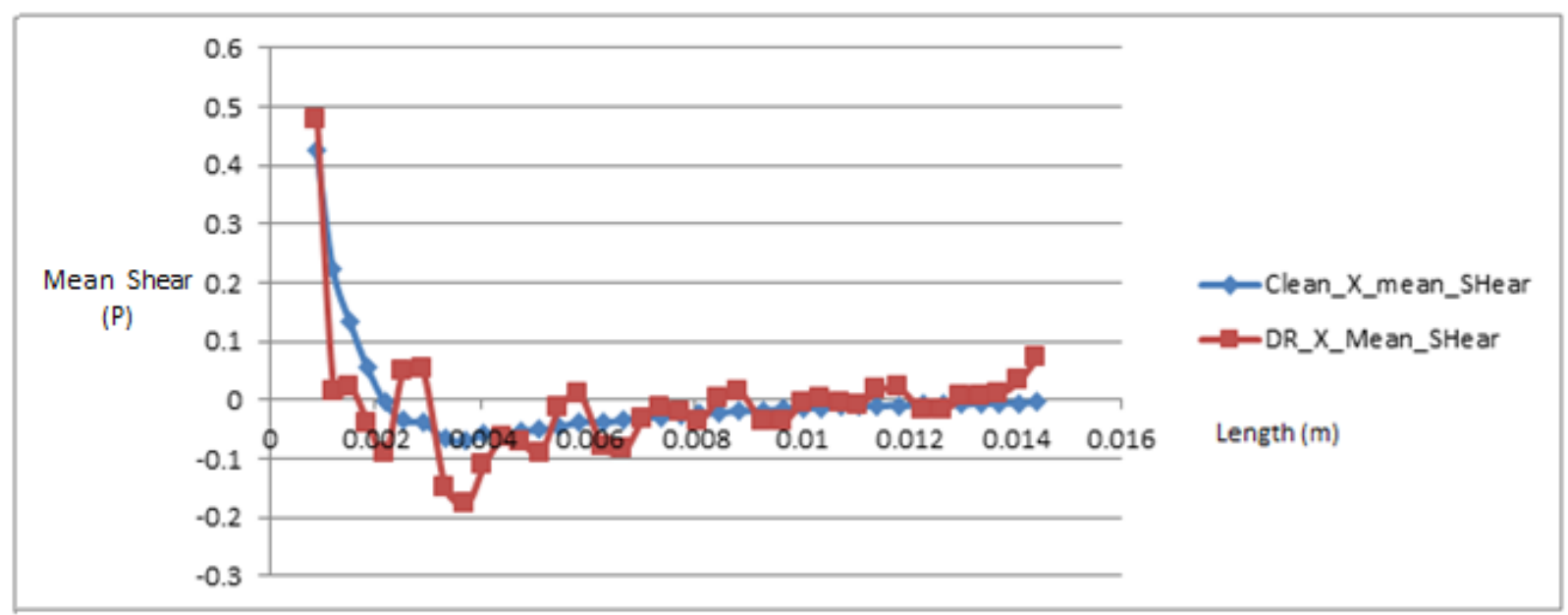

Figure 4.11 Mean wall shear stress from simulations 


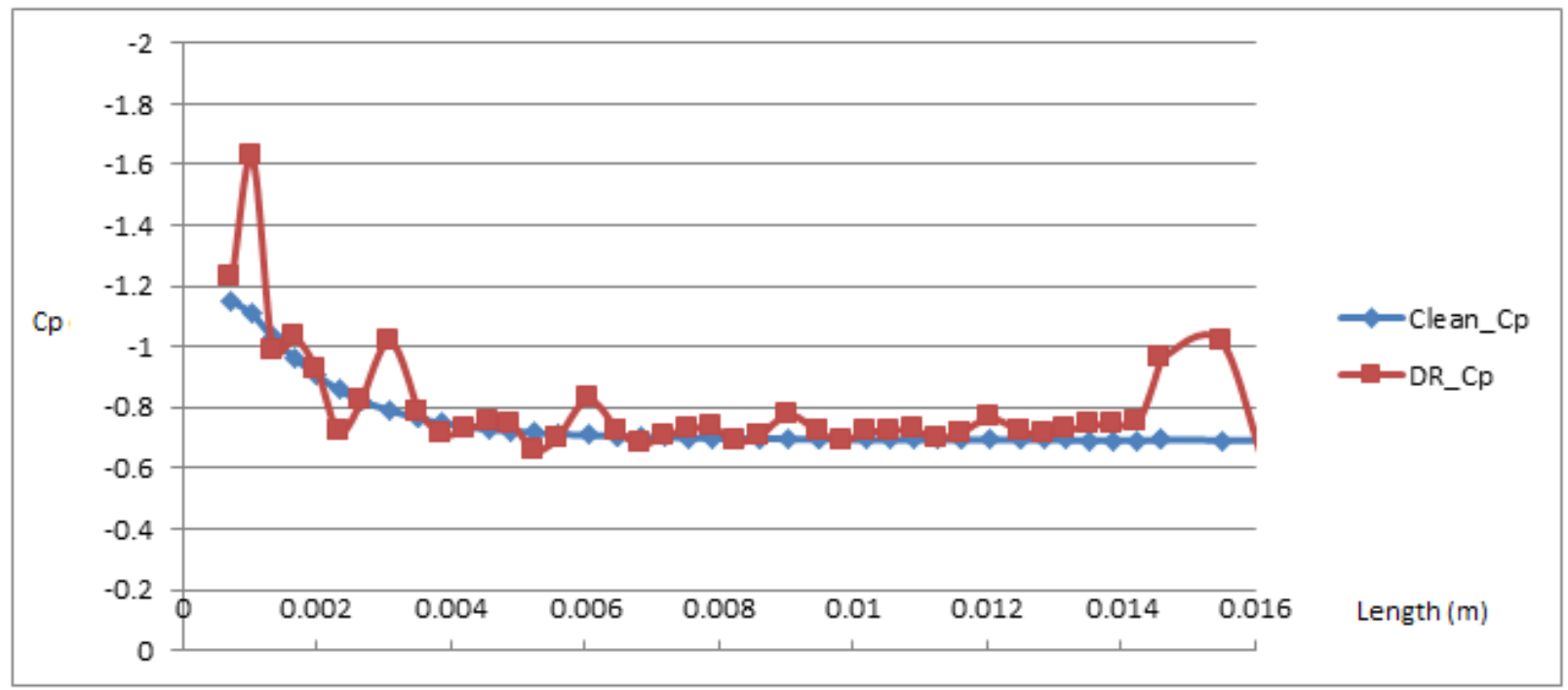

Figure 4.12 Mean Cp data over DR region from simulations

\subsection{Additional Results - Studies for Reynolds Number of 25000}

Numerical studies have also been performed on flow control over an airfoil at a Reynolds number of 25000. Grids have been developed for angles of attack of $10^{\circ}$ and $13^{\circ}$ at this Reynolds number. These grids have only 10 elements in the span wise direction. The numbers of cells over a hump are 10 elements in either direction. The grid size was around a total of 1.6 million cells. The time step size for these runs is $7 \times 10^{-6} \mathrm{~s}$. A clean run has been performed for 20 flow through times for both angle of attack values. Then it was time averaged for another 10 flow through times. The separation point for the clean run is at $2 \%$ c. The DR case was started using the clean case at 20 flow through times as the initial condition and was run for 10 flow through times, and then averaged for another 10 flow through times. The DR here has again been actuated at a frequency of $90 \mathrm{~Hz}$ with a maximum amplitude of 230 microns. All the parameters are the same as before for the following runs. Figure 4-13 shows the comparison of velocity magnitude contours for the clean case in this study with experimental PIV data (10). The PIV data is shown on the left hand side and CFD data on the right hand side. It can be seen that there is a good overall match between the experimental and CFD data qualitatively. Leading edge stall 
can be clearly seen from both sets of data. The results show a very close match at both angles of attack. For the DR case, the study has been only performed at an angle of attack of 10 degrees. Figure 4-14 shows the comparison of velocity magnitude contours of experimental results and CFD results for the DR case. DR tries to reattach the flow but there is a big recirculation region and the stall continues. The lower the Reynolds number, the more difficult is the flow control operation. The higher the Reynolds number, the easier it is to control the flow.
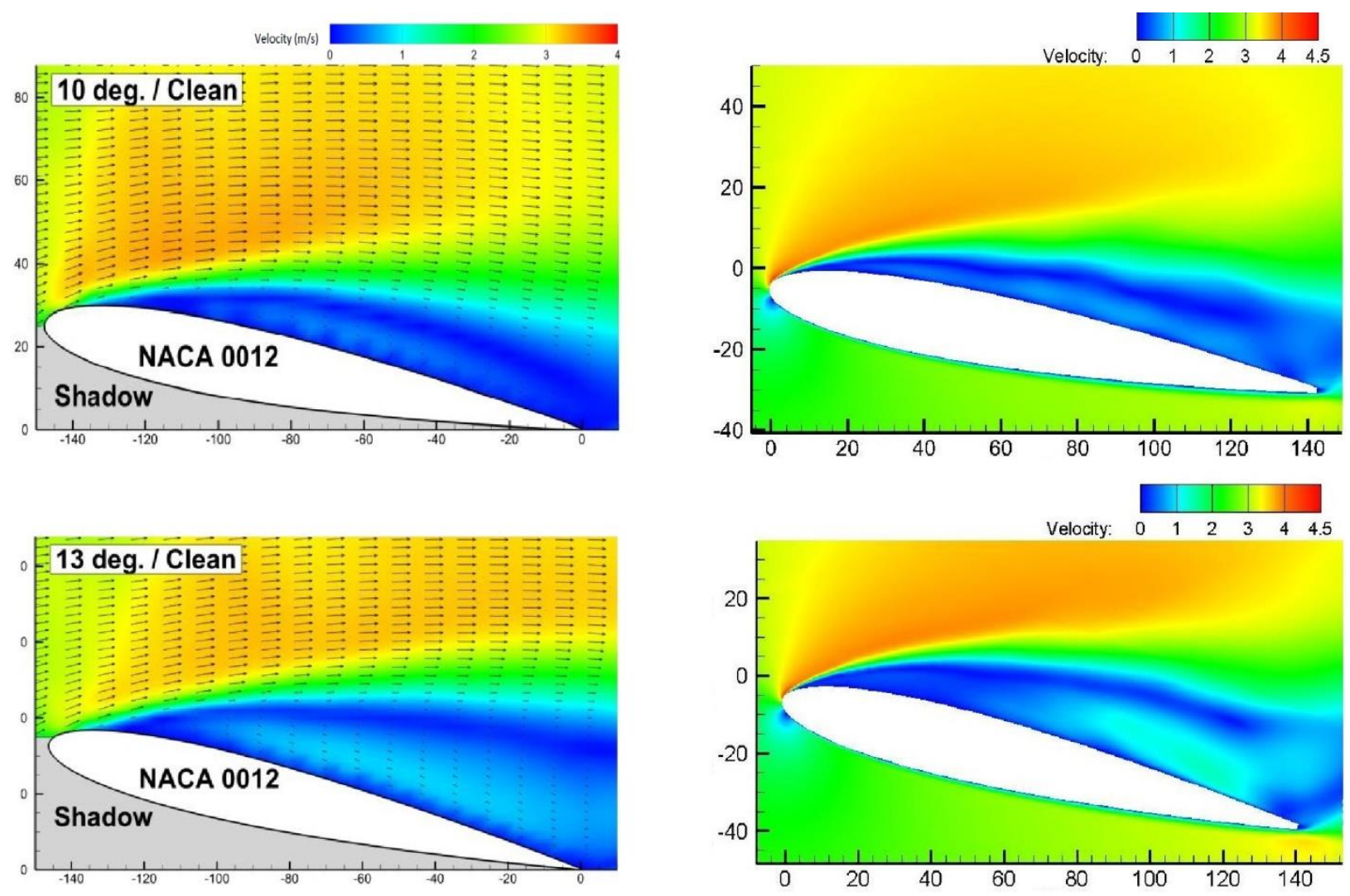

Experimental results (10)

\section{CFD results}

Figure 4.13 Comparison of velocity magnitude contours for clean case at $\mathbf{R e} \mathbf{2 5 0 0 0}$ 


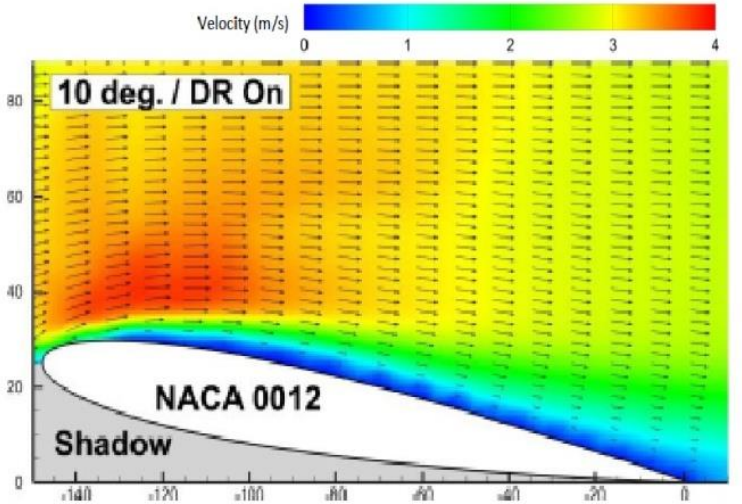

Experimental results (10)

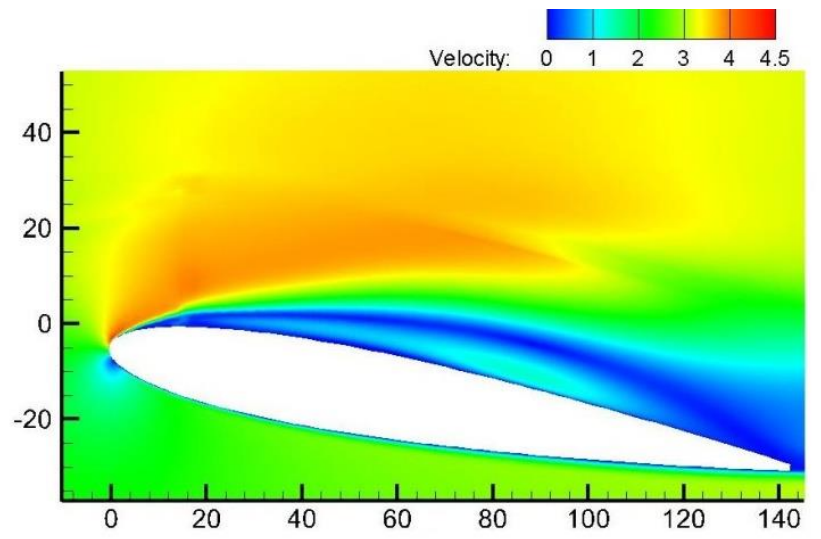

CFD results

Figure 4.14 Comparison of velocity magnitude contours for DR case at Re 25000

The saw-tooth like profile is observed in the DR velocity data as whenever DR is in operation, the humps oscillate with a certain frequency. So in their operation, they energize the flow by causing mixing and entrainment. They continuously attach the separated layer. That is why we see the saw tooth like structure in the velocity data as a result of the frequency in operation.

\subsection{Additional Results - Study on Order of Transient Formulation}

Second order transient formulation is necessary for accuracy in LES simulations. In this study, the clean cases have been run with second order implicit transient formulation. However the DR cases have been run with first order implicit transient formulation. This is because the dynamic meshing in FLUENT does not allow for second order transient formulation. In the process to find the likely causes of failure of DR mechanism, to effectively control the flow in this study, a separate study has been performed to determine the effect of the order of transient formulation on the flow. This was performed on an airfoil at an angle of attack of 14 degrees at a Reynolds number of 49000. A clean run has been performed with first order transient formulation for 20 flow through times and then it was time averaged for another 10 flow through times. The results 
have been compared to the results from experiments and the results from simulation with second order transient formulation. Figure 4-15 shows the comparison of velocity magnitude contours of experimental results and CFD results for both the transient formulation cases. It is evident from these plots that the velocity magnitude contours show a better match for the second order case with the experiments compared to the first order. Recirculation zone appears to be larger with $1^{\text {st }}$ order case. 


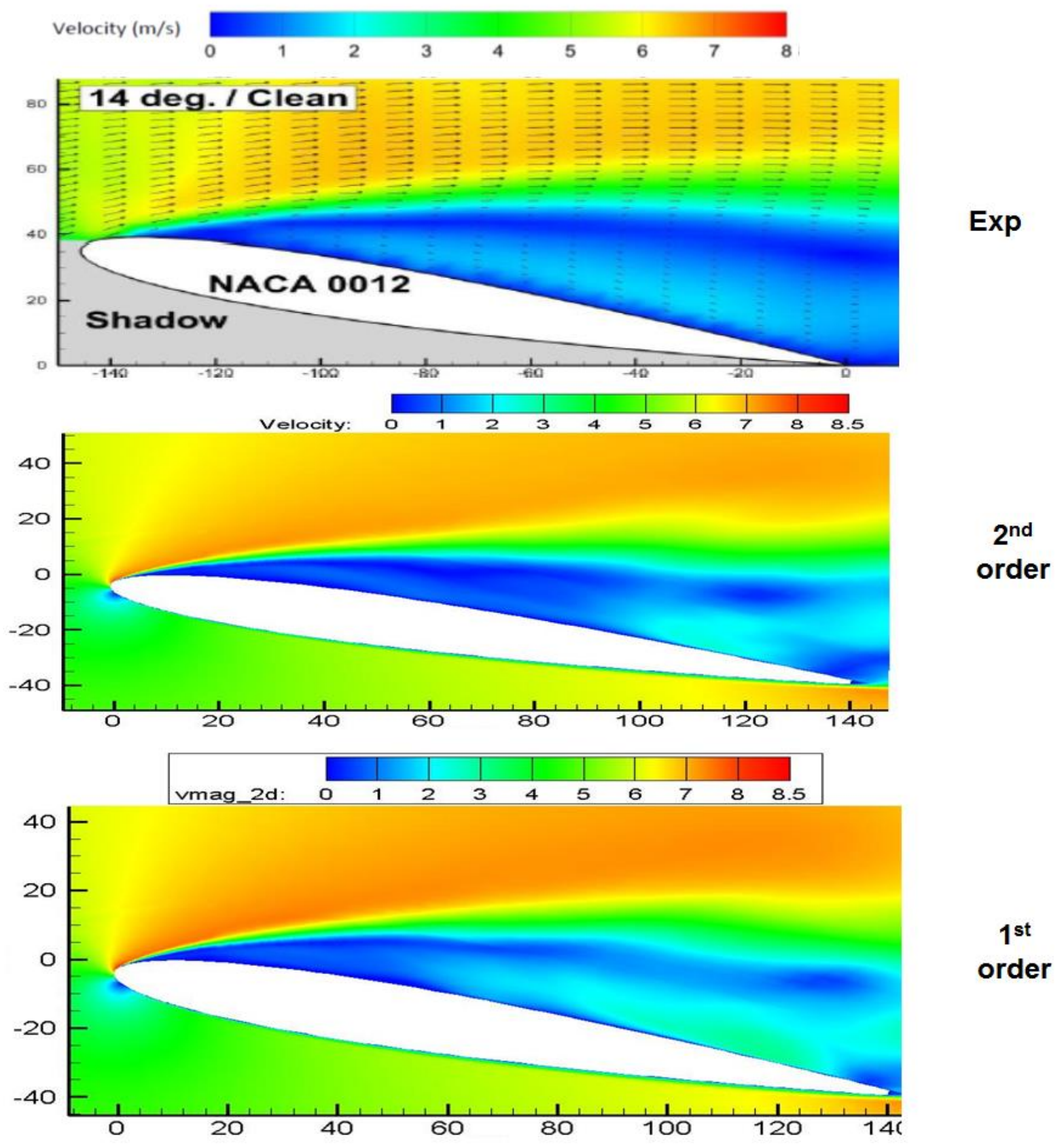

Figure 4.15 Comparison of velocity magnitude contours for clean cases at $\mathbf{R e} 49000$ 
Figure 4-16 shows the comparison of TKE contours of experimental results and CFD results for both the transient formulation cases.

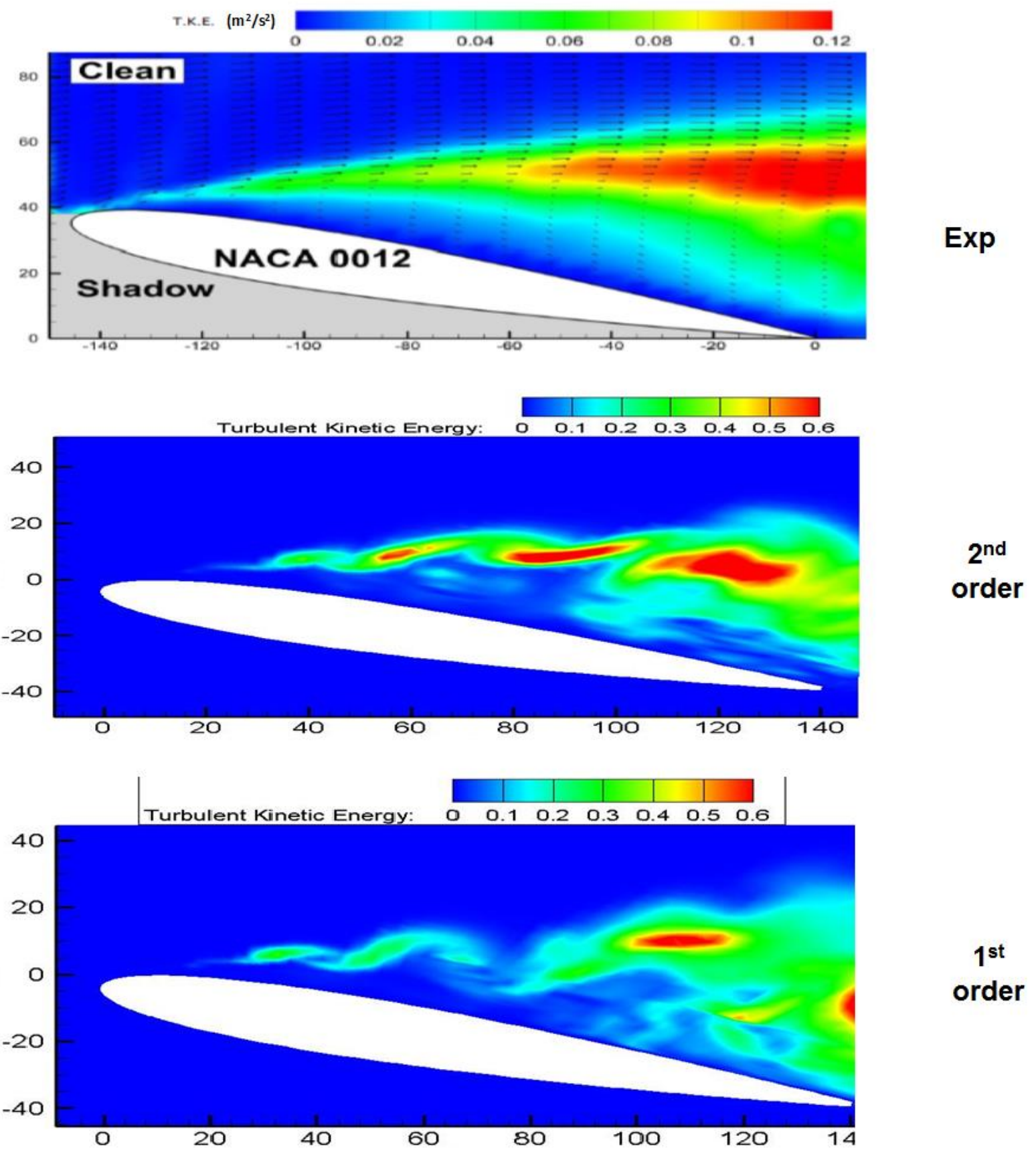

Figure 4.16 Comparison of TKE contours for clean cases at $\operatorname{Re} 49000$ 
TKE contour of the second order case matches experiments better qualitatively compared to first order. Figure 4-17 shows the comparison of vorticity contours of experimental results and CFD results for both the transient formulation cases. The vorticity pattern of experiments has a better match with second order case especially the separation pattern from the leading edge. In the first
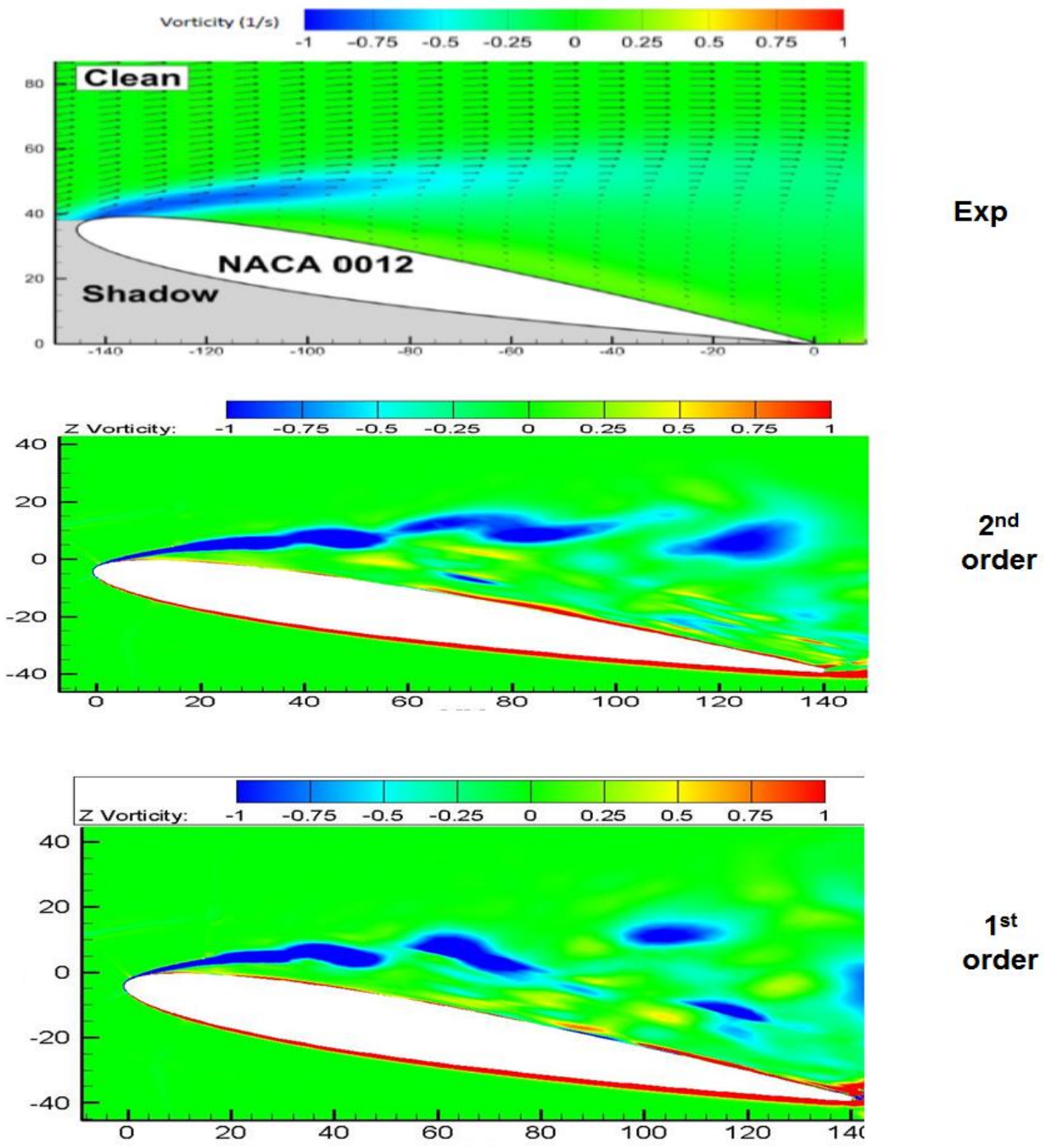

Figure 4.17 Comparison of vorticity contours for clean cases at $\mathbf{R e} 49000$ 
order case there is a discontinuity in the pattern and it lowers slightly down at about mid chord.

Figure 4-18 shows the comparison of mean x-wall shear stress for both the transient formulation cases in simulations. The separation point is at 1.3 percent chord length for second order case and around 1.1 percent chord length for first order case. Based on the above analysis, there is a definite effect of order of transient formulation on results. It is clearly evident that this might be one of the possible causes for failure of DR in effectively controlling flow separation.
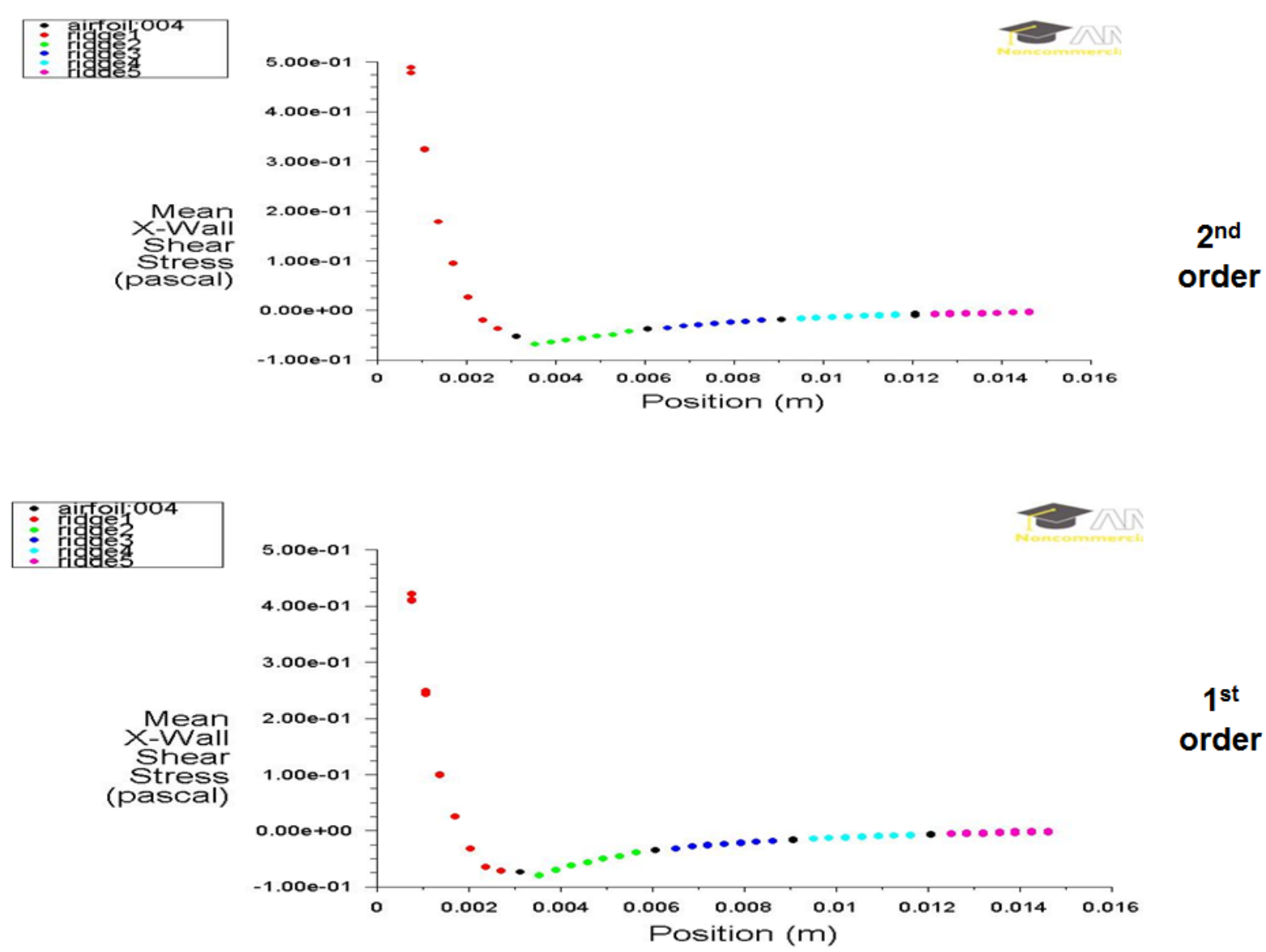

Figure 4.18 Comparison of mean x-wall shear stress for clean cases at $\operatorname{Re} 49000$ 


\subsection{Additional Results - Study with higher amplitude}

Simulation has been performed by changing amplitude from $0.23 \mathrm{~mm}$ to $0.5 \mathrm{~mm}$. This has been done for the airfoil at an angle of attack of 10 degrees at a Reynolds number of 25000 . The figure below shows the comparison of velocity magnitude contours for the Clean and DR case.

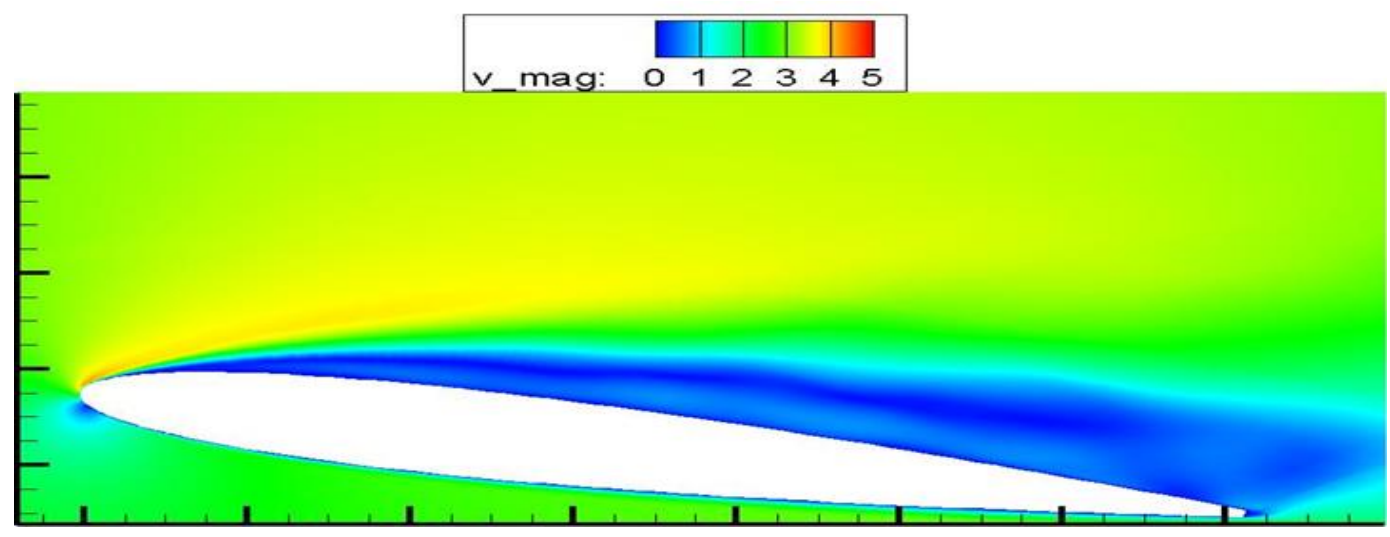

a) Velocity magnitude contour for clean case

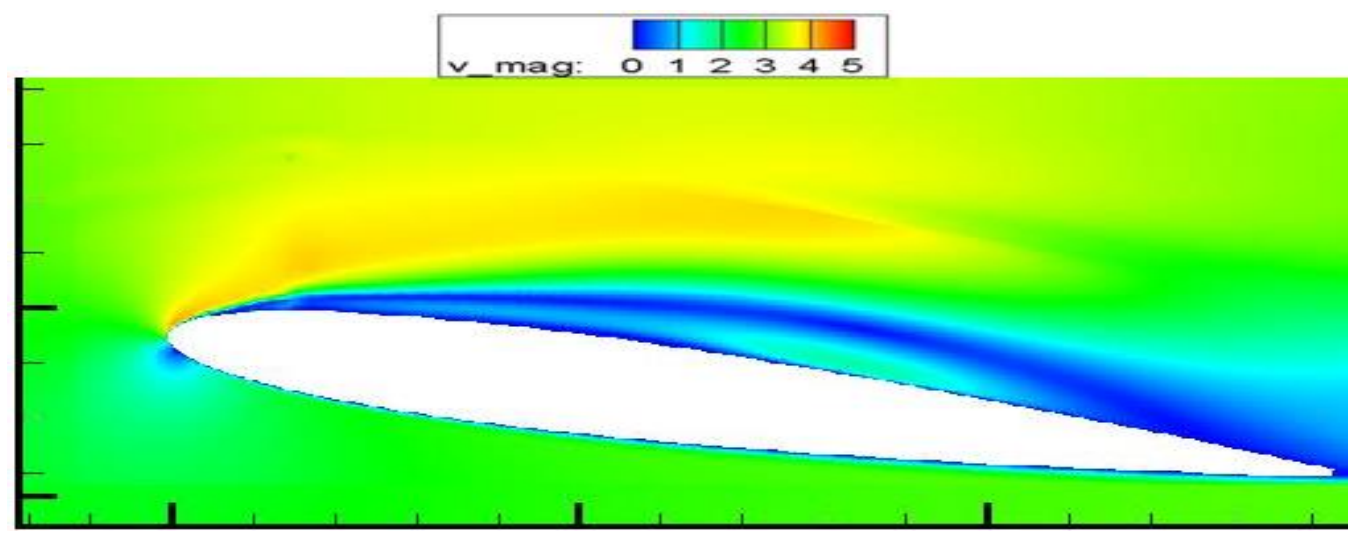

b) Velocity magnitude contour for clean case

Figure 4.19 Comparison of velocity magnitude contours for Re 25000 for DR amplitude of $0.5 \mathrm{~mm}$

It is clearly evident from the above figure that flow control is not observed and there is recirculation observed and stall continues. DR by principle is very effective when the amplitude is below the boundary layer height. In this case, the boundary layer height is about $0.235 \mathrm{~mm}$. With an amplitude of $0.5 \mathrm{~mm}$, the humps protrude above the boundary layer. 


\section{Conclusions}

Numerical studies have been performed to investigate the effect of dynamic roughness on the separated flow over an airfoil at a Reynolds numbers of 49000 and 25000. A large eddy simulation has been performed over a NACA 0012 airfoil. The airfoil is inclined at an angle of $14^{\circ}$ in the study at $\operatorname{Re}=49000$ and $10^{\circ}$ and $13^{\circ}$ in the study at $\operatorname{Re}=25000$, based on experiments of Grager (10). The simulations have been performed using the commercial CFD software Ansys Fluent version 13.0. All the simulations have been run on the WVU MOUNTAINEER cluster. The motion of 3-D dynamic roughness humps was done by using a UDF. The DR has been actuated at $90 \mathrm{~Hz}$ with a maximum amplitude of 230 microns in both the cases, again to match the experimental conditions. The results are compared with the experimental PIV work (10). The results of this study can be summarized as follows:

- The leading edge stall behavior has been well captured for both the Reynolds numbers. For the clean cases, the LES was able to reproduce experimental results qualitatively in a reasonable fashion.

- In this study DR tries to reattach the separated flow in the simulations but no control is achieved compared to the experimental data for both the Reynolds numbers.

- There is a high concentration of vorticity in the simulations near the DR region indicating that the vortices are generated by DR which results in the mixing phenomenon, justifying the principle behind the effectiveness of DR.

- Several possible external factors have been identified which might have assisted the flow control in experiments which the simulations are not taking into account. This might be the reason behind the failure of DR to control the flow in this study. 
- DR reduced the height of the separated layer in the simulations relative to the corresponding clean case.

- This study introduces a new method of transformation of ridges to humps making meshing much simpler and quicker. By this technique, ridge surfaces can transformed into desired hump patterns by just modifying the hump geometry-defining equations. The developed UDF can be altered easily to suit the required hump pattern.

- The computed separation point location moves upstream as the Reynolds number is increased. The separation point for the $\mathrm{Re}=25000$ was at $2 \% \mathrm{c}$ and that for $\mathrm{Re}=49000$ was $1.3 \% \mathrm{c}$.

- By application of DR, an increase of $2.7 \%$ in L/D has been obtained.

- The order of transient formulation used has an effect on the flow. In the clean case, the second order transient simulation results show good comparison with PIV data qualitatively compared to the first order case. Since the dynamic meshing with FLUENT is only possible with first order method, it is likely the reason behind failure of DR in controlling the flow in simulations.

- Increasing the amplitude of humps above the boundary layer height in the simulations does not have an effect on the flow control as confirmed by literature. 


\section{Recommendations}

From the above numerical study, it can be observed that DR could not effectively control the flow but a lot of potential benefits have been observed. Total flow control was the aim but the study fell short of it due to various limitations. The following recommendations are suggested:

- The ridge to hump transformation strategy can be effectively used in numerical studies involving dynamic roughness. The above mentioned technique saves lot of time and reduces the burden of meshing individual hump elements.

- Second order transient formulation must be used for Large Eddy Simulations in order to get accurate results.

- Though there is an increased computational cost, the meshes should be well resolved in the stream wise direction.

- Cell count refinement option of ICEM can be used to manipulate the mesh size in Z direction in order to keep the grid size within bounds.

- Other CFD packages like OPENFOAM can be tried to run LES with DR with second order transient formulation as FLUENT does not support this with dynamic meshing. 


\section{References}

1. Low Reynolds Number Airfoil Survey. Carmichael, B.H. 1981, NASA CR 165803, Vol. 1.

2. Gad-El-Hak, M. Flow Control : Passive, Active and Reactive Flow Management. s.I. : Cambridge University Press, 2000.

3. McCullogh, G.B and Gault, D.E. Examples of Three Different Types of Airfoil-Section Stall at Low Speeds. National Advisory Committee for Aeronautics. Washington : s.n., 1951.

4. The Control of Flow Separation by Periodic Excitation. Greenblatt, D and Wygnanski, I.J. 2000, Proogress in Aerospace Sciences, Vol. 36, pp. 487-545.

5. Two-Dimensional Simulation of Dynamic Surface Roughness for Aerodynamic Flow Control. Huebsch, W.W. 2, March-April 2006, Journal of Aircraft, Vol. 43, pp. 353-362.

6. Dynamic Roughness as a Means of Leading-Edge Separation Flow Control . Huebsch, W. W, Hamburg, P. D and Rothmayer, A. P. 1, January-February 2012, Journal of Aircraft, Vol. 49.

7. Low Reynolds Number Stall Suppression with Dynamic Roughness. Grager, T, et al. New Orleans : s.n., 2012. 6th AIAA Flow Control Conference.

8. Gall, P. D. A Numerical and Experimental Study of the Effects of Dynamic Roughness on Laminar Leading Edge Separation. West Virginia University. Morgantown : s.n., 2010. PhD Dissertation.

9. Investigation of Dynamic roughness Flow Control on NACA 0012 Airfoil at Low Reynolds Number. Jakkali, V, et al. Montreal : s.n., 2013, SAE Aerotech Congress and Exhibition.

10. Grager, T. Experimental Study of Low Reynolds Number Flow Control Devices : Dynamic Burst Control Plate and Dynamic Roughness. Ames : s.n., 2011. M.S. Thesis.

11. Separated Shear Layer Transition over an Airfoil at a Low Reynolds Number. Boutilier, M. S.H. and Yarusevych, S. 24, 2012, Physics of Fluids. 084105.

12. Kim, S-E. Simulation of Turbulent Flows -LES \& DES with FLUENT. 2004.

13. Large eddy and Reynolds-Averaged Navier-Stokes Simulations of Turbulent Flow over an Airfoil. Weber, C. and Ducros, F. 4, 2007, International Journal of Computational Fluid Dynamics, Vol. 13, pp. 327-355.

14. Large Eddy Simulations: Achievements and Challenges. Piomelli, U. 4, 1999, Progress in Aerospace Sciences, Vol. 35, pp. 335-362.

15. Intermittent Bursting of a Laminar Separation Bubble on an Airfoil. Almutairi, J. H., Jones, L. E. and Sandham, N. D. 2, February 2010, AiAA journal, Vol. 48. 
16. Large-Eddy Simulation of Low-Reynolds Number Over Thick and Thin NACA Airfoils. Kojima, R., et al. 1, January-February 2013, Journal of Aircraft, Vol. 50.

17. Large-Eddy Simulation of Transition in a Separation Bubble. Roberts, S. K. and Yaras, M. I. March 2006, Transactions of ASME, Vol. 128, pp. 232-238.

18. Smagorinsky, J. General Criculation Experiments with the Primitive Equations. I. The Basic Experiment. 1963. pp. 99-164. 91.

19. A Dynamic Subgrid-Scale Eddy Viscosity Model. Germano, M., et al. 7, July 1991, Physics of fluids, Vol. 3.

20. A Proposed Modification of the Germano Subgrid-Scale Closure Method. Lilly, D. K. 3, March 1992, Physics of Fluids, Vol. 4.

21. Subgrid-Scale Stress Modelling based on the Square of the Velocity Gradient Tensor, Flow, Turbulence and Combustion. Nicoud, F. and Ducros, F. 3, 1999, Vol. 62, pp. 183-200.

22. Application of the Localized Dynamic Subgrid-Scale Model to Turbulent Wall-Bounded Flows. Kim, W. W. and Menon, S. Reno : s.n., January 6-9, 1997, AIAA.

23. Versteeg, H. K. and Malalasekara, W. An Introduction to Computational Fluid Dynamics, the Finite Volume Method. Harlow : Pearson Education Limited, 2007.

24. Mathieu, J. and Scott, J. An Introduction to Turbulent Flow. 1. 2000.

25. www.cfd-online.com. CFD Online website. [Online]

26. Fluent Theory Guide. [Version 13.0] Lebanon, NH : s.n., 2005.

27. Flow Control : The Future. Gad-El-Hak, M. 3, 2001, Journal of Aircraft, Vol. 3.

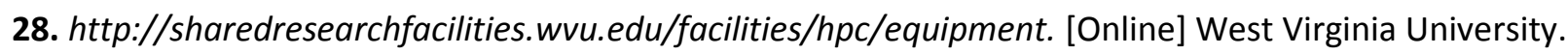




\section{Appendix}

\section{UDF for humps - Re 49000}

Five grid motion subroutines have been used for moving the five row of humps. Each subroutine differs only in the specification of hump location and number of humps along the airfoil. The DR humps are in a staggered pattern.

\footnotetext{
/***************************************************************************/

/* true sine hump UDF by Pete Gall

$* /$

/* modified by Chris Griffin 8-20-2012

/* modified by Venkata Subba Sai Satish Guda 9-04-2012

$* 1$

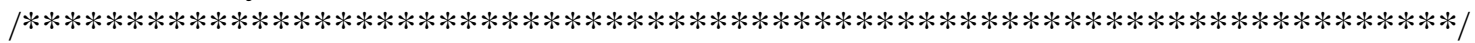

\#include "udf.h"

\#define omega $1.5 / *$ rotational speed, $\mathrm{rad} / \mathrm{sec} \quad * /$

\#define pi 3.14159265

static real chord $=0.15 ; \quad / *$ chord length in meters $\quad * /$

static real amp $=0.00023 ; \quad / *$ amplitude in meters*/

static real humpdia $=0.00212 ; \quad / *$ hump diameter in meters $* /$

static real humpgap $=0.00226 ; \quad / *$ gap between humps in meters */

/****** First row hump motion $* * * * * * /$

DEFINE_GRID_MOTION(moving_arc_a01, domain, dt, time, dtime)

\{

Thread $*$ tf $=$ DT_THREAD $(\mathrm{dt})$;

face_t $\mathrm{f}$;

Node *node_p;

real alpha, theta, $x$, phi, gamma, y,z, x1, x2, y1, y2, lpln, yfh, ln;

real ymag, z1,zmod, lp, dx, dy;

int n,numb,zumb;

/* Set/activate the deforming flag on adjacent cell zone, which */

$/ *$ means that the cells adjacent to the deforming wall will also be */

/* deformed, in order to avoid skewness. */

SET_DEFORMING_THREAD_FLAG (THREAD_T0 (tf));

/* Compute the angles:

$* /$

alpha $=2.0 *$ omega $* 94.0 *$ CURRENT_TIME;

$\mathrm{x} 1=0.00074162707$;
} 
else if $(z<=-0.0065 \& \mathrm{z}>=-0.00862)$

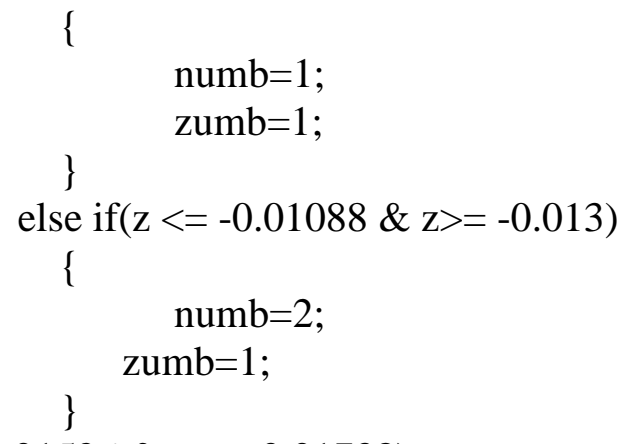

zmod $=\sin (((((-$ numb $*$ humpgap $)-$

z)/humpdia $)^{*}$ pi $)+((\text { numb+1)*pi }))^{*}$ zumb;

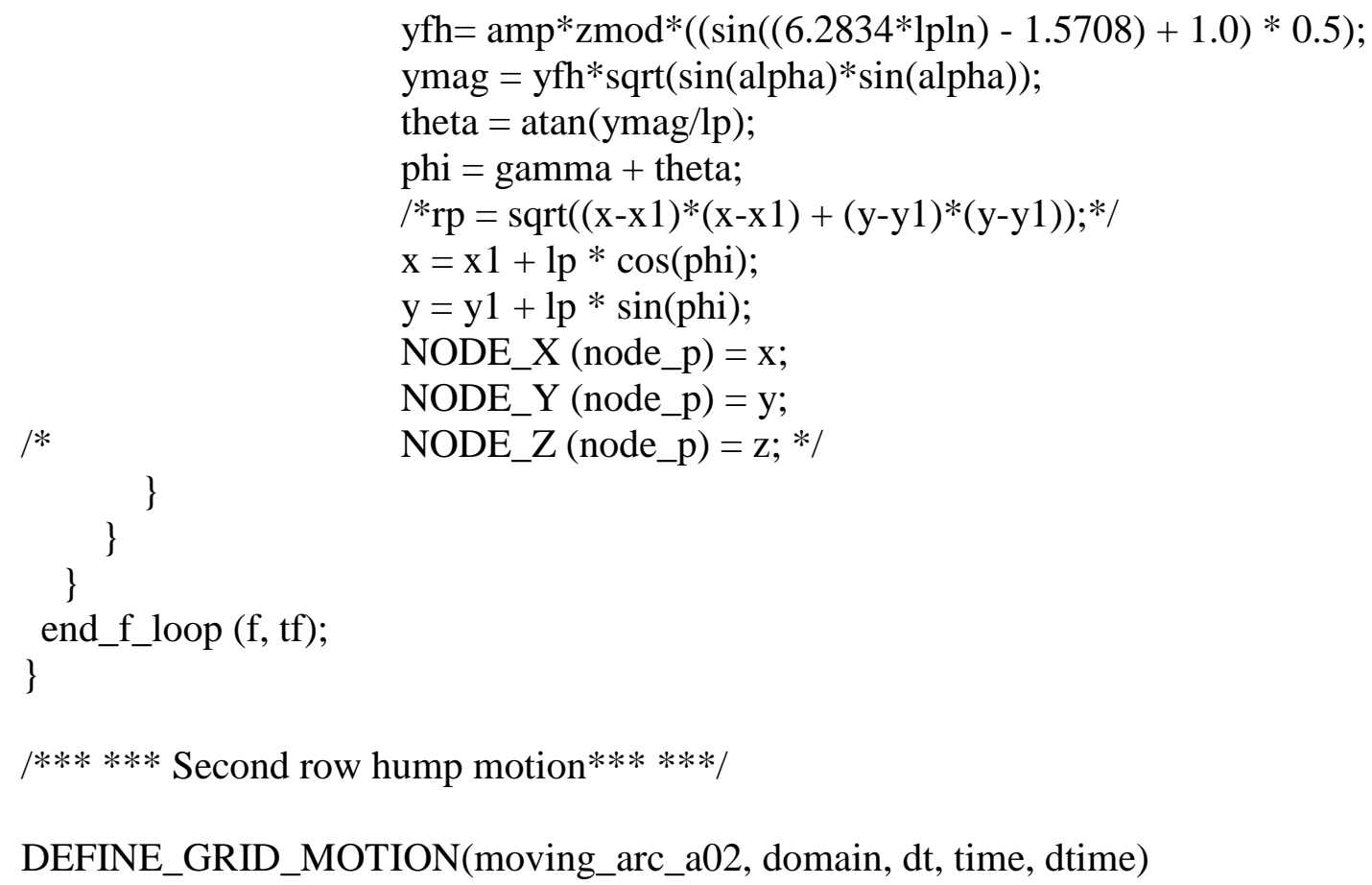




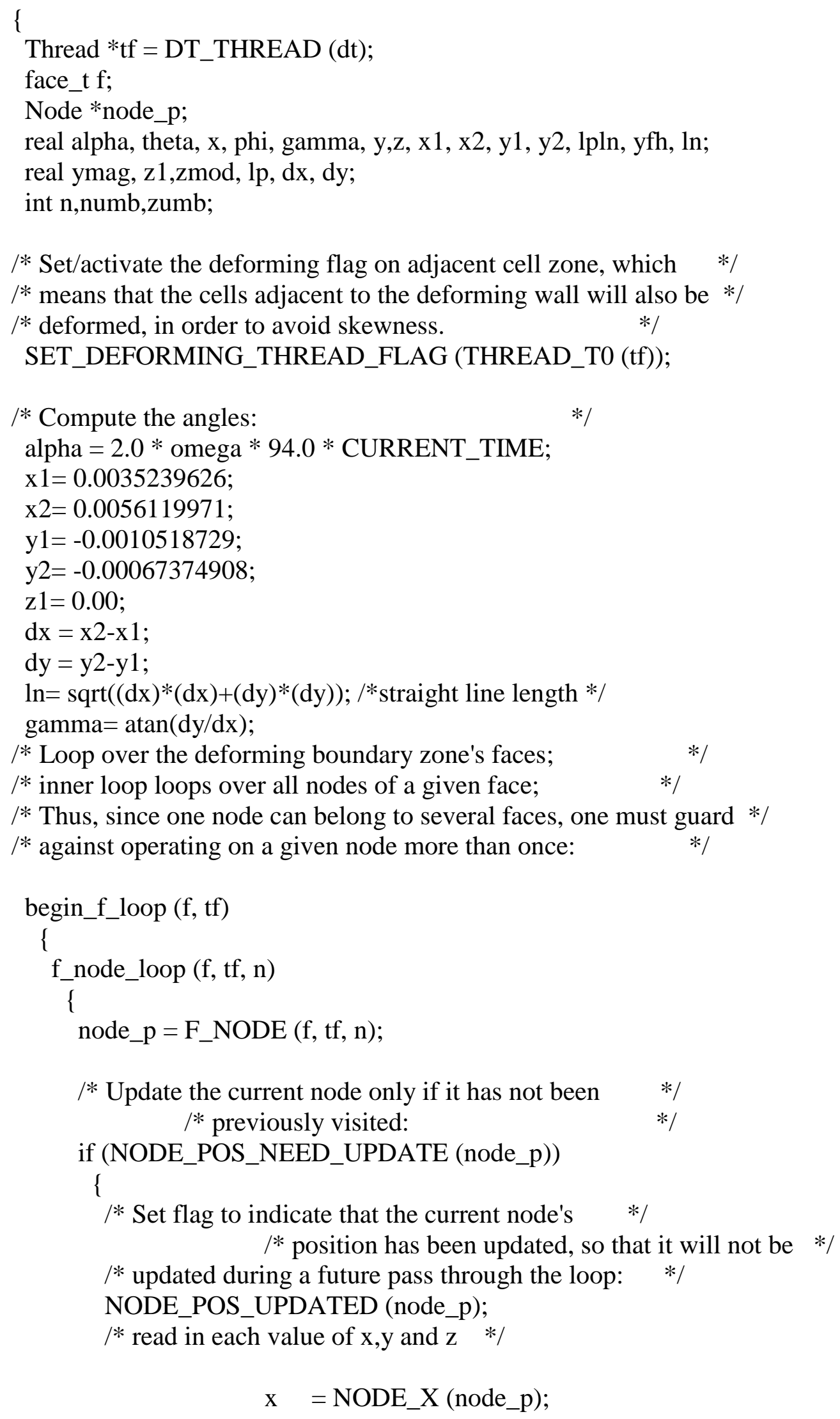




$$
\begin{aligned}
& \mathrm{y}=\mathrm{NODE} \_\mathrm{Y}(\text { node_p}) ; \\
& \mathrm{z}=\mathrm{NODE} \mathrm{Z} \text { (node_p); } \\
& \quad / * \text { lpln is normalized distance of each point along the hypotinuse of the }
\end{aligned}
$$

line $* /$

$$
\begin{aligned}
& \mathrm{lp}=\operatorname{sqrt}\left((\mathrm{x}-\mathrm{x} 1)^{*}(\mathrm{x}-\mathrm{x} 1)+(\mathrm{y}-\mathrm{y} 1)^{*}(\mathrm{y}-\mathrm{y} 1)\right) ; \\
& \mathrm{lpln}=\operatorname{sqrt}\left((\mathrm{x}-\mathrm{x} 1)^{*}(\mathrm{x}-\mathrm{x} 1)+(\mathrm{y}-\mathrm{y} 1)^{*}(\mathrm{y}-\mathrm{y} 1)\right) / \mathrm{ln} ;
\end{aligned}
$$

$/ *$ zmod will be used to calculate the tapering off of the y magnitude at the ends of the hump in the $\mathrm{z}$ direction*/

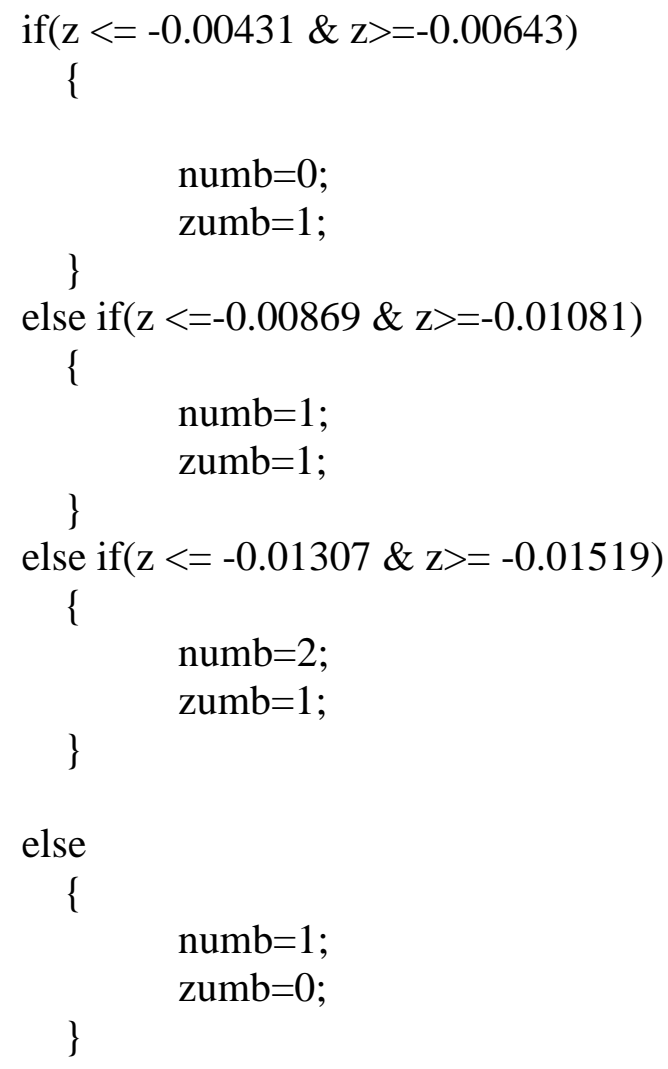

zmod $=\sin ((((-0.00431-($ numb*humpgap $)-$

z)/humpdia)*pi)+((numb)*pi))*zumb;

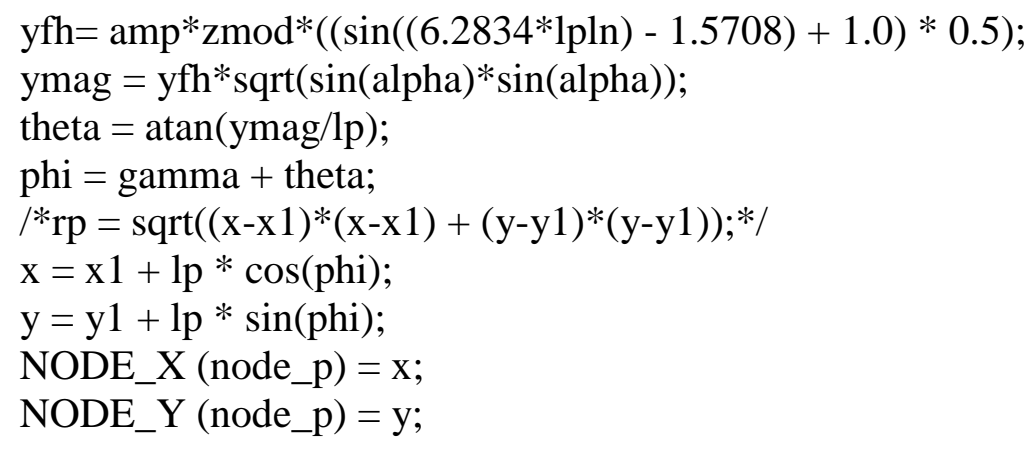




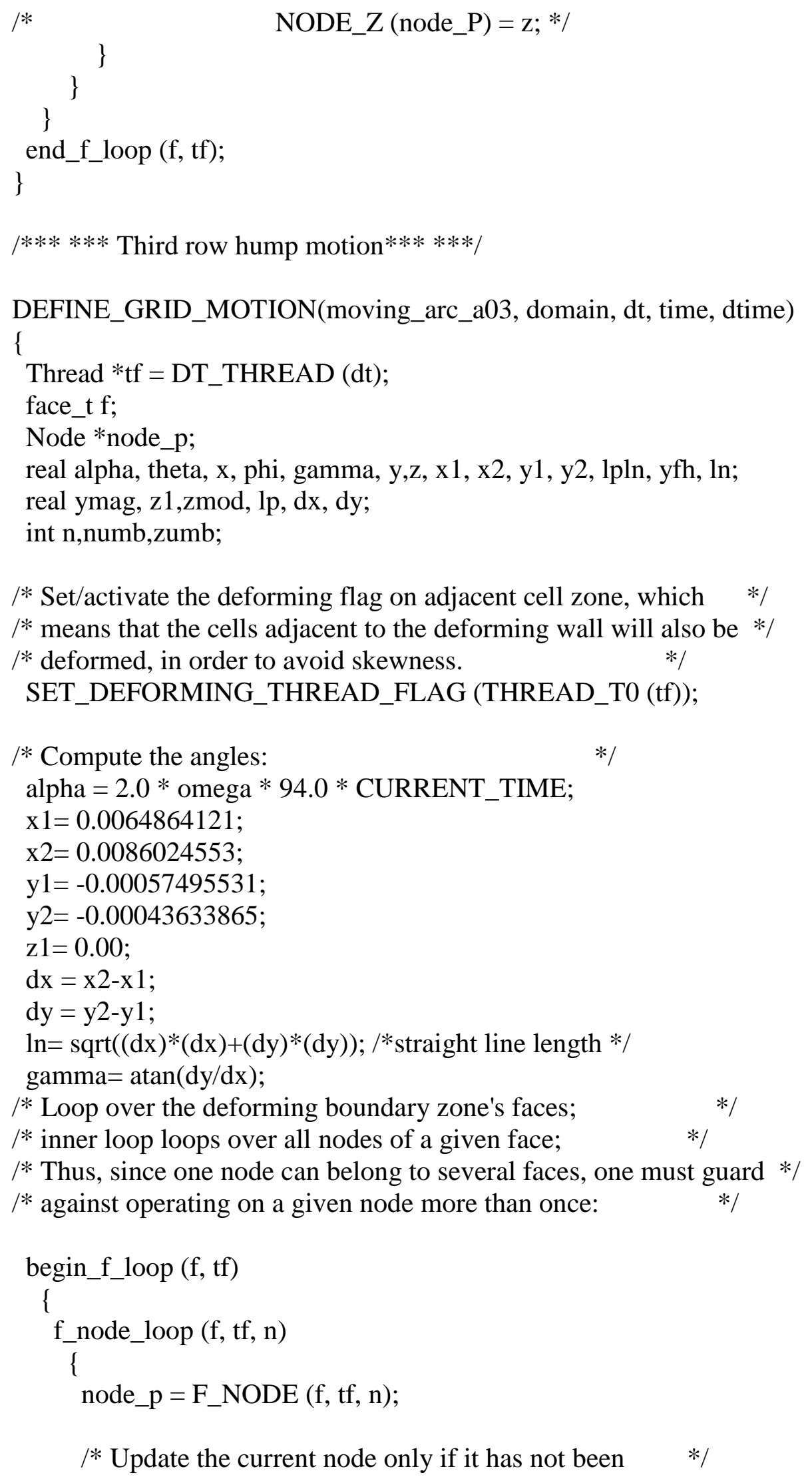




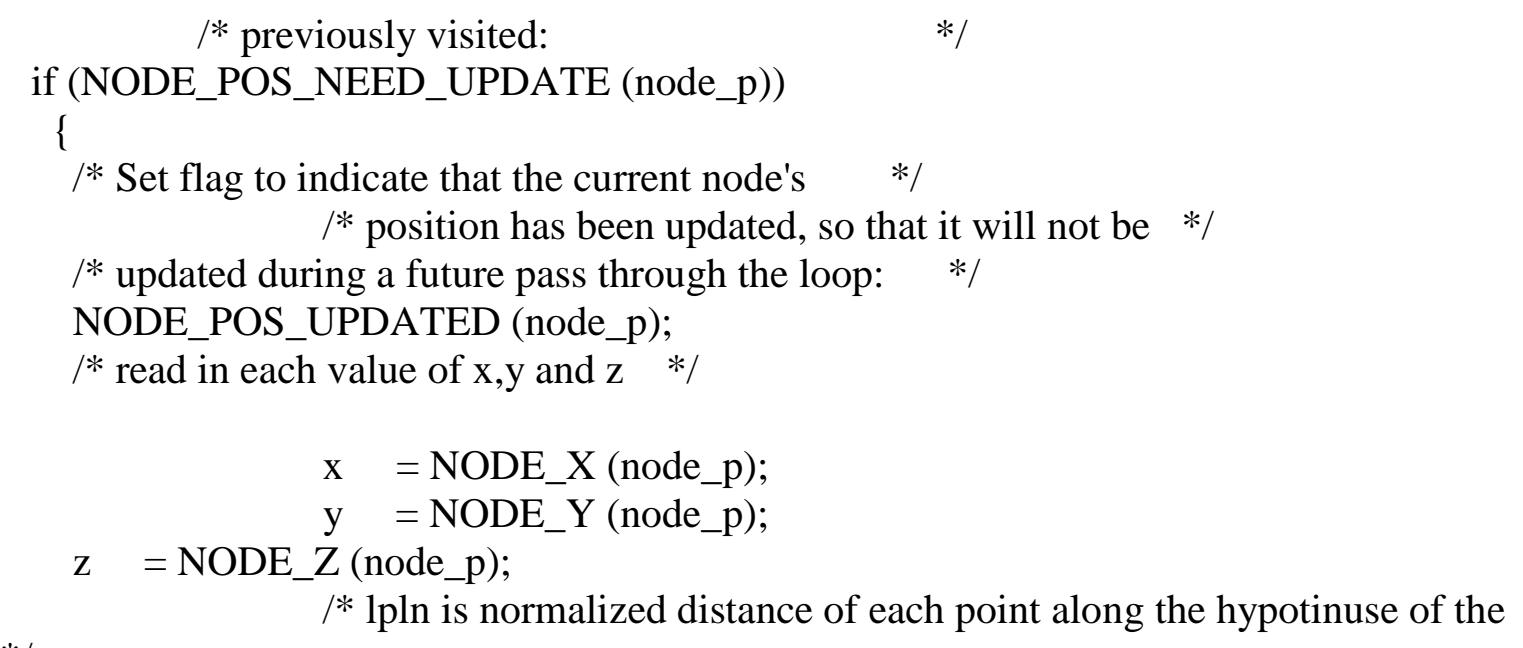

line */

$$
\begin{aligned}
& \operatorname{lp}=\operatorname{sqrt}\left((x-x 1)^{*}(x-x 1)+(y-y 1)^{*}(y-y 1)\right) \\
& \operatorname{lpln}=\operatorname{sqrt}\left((x-x 1)^{*}(x-x 1)+(y-y 1)^{*}(y-y 1)\right) / 1 n
\end{aligned}
$$

$/^{*}$ zmod will be used to calculate the tapering off of the $y$ magnitude at the ends of the hump in the $\mathrm{z}$ direction*/

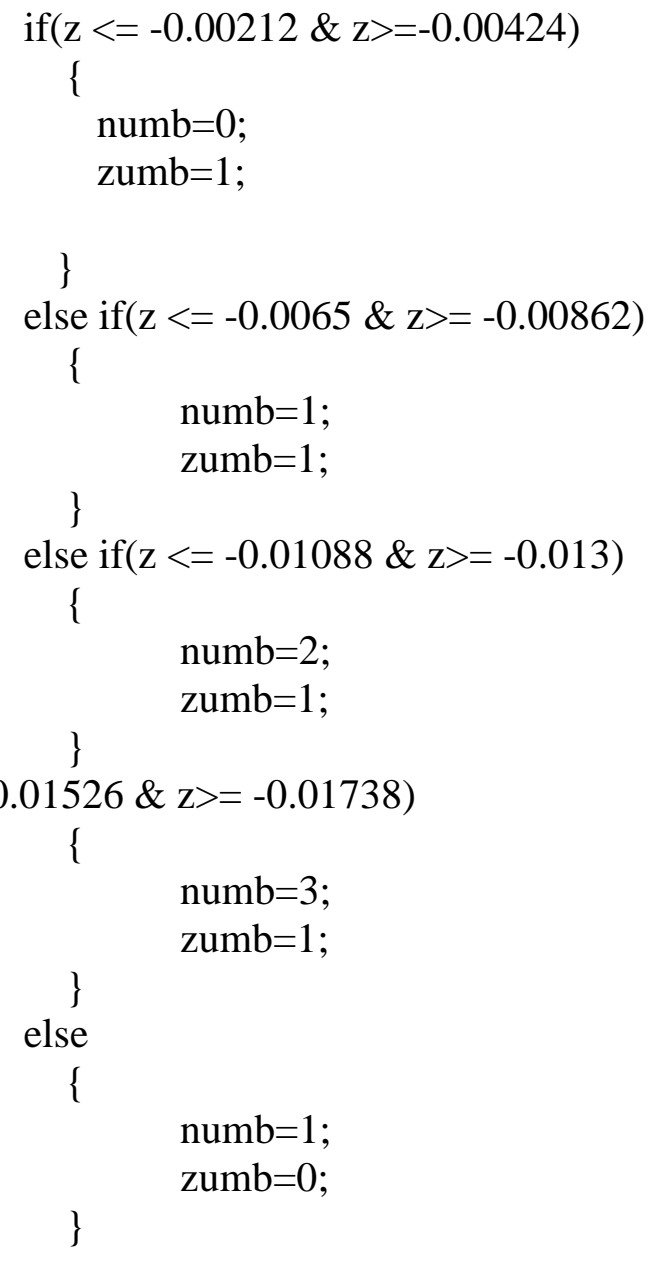


z)/humpdia)*pi)+((numb+1)*pi))*zumb;

$$
\text { zmod }=\sin (((((-n u m b * \text { humpgap })-
$$

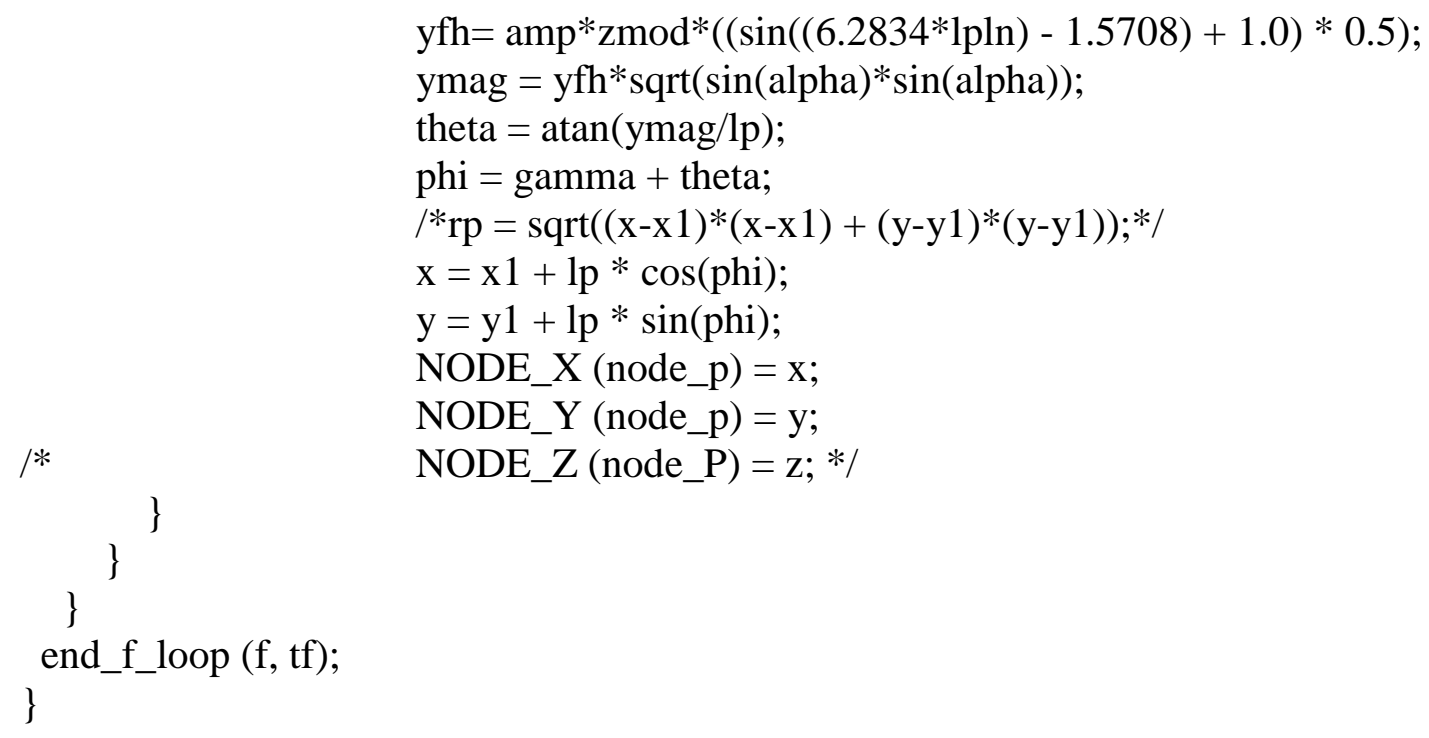




$$
\begin{array}{cc}
\text { numb=2; } \\
\text { zumb=1; } \\
\text { else } & \\
\{ & \\
& \text { numb=1; } \\
\text { zumb }=0 ;
\end{array}
$$

$\mathrm{zmod}=\sin ((((-0.00431-($ numb*humpgap $)-$

z)/humpdia $)^{*}$ pi $)+(($ numb $) *$ pi $\left.)\right)^{*}$ zumb;

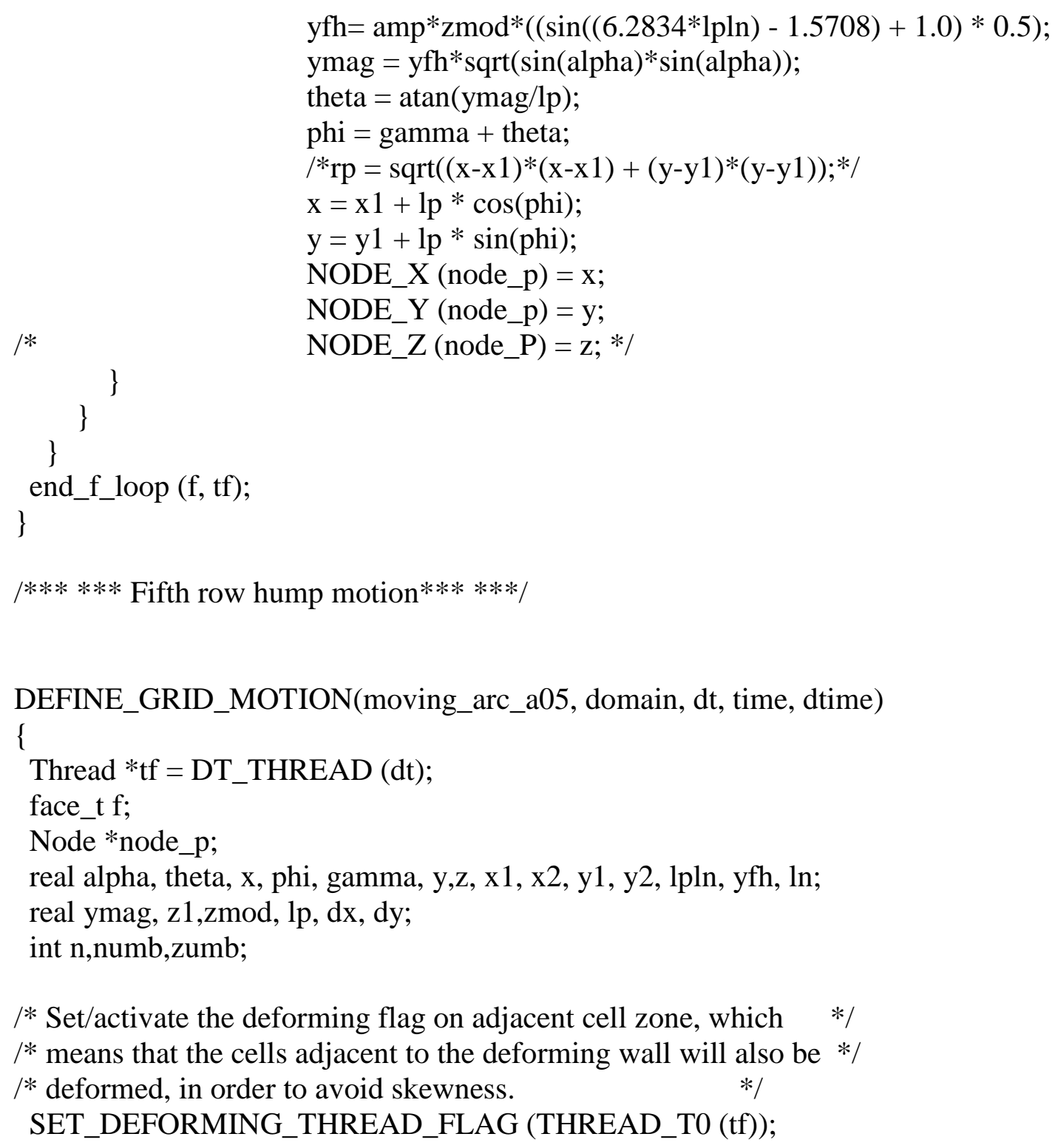




$$
\begin{aligned}
& \text { numb=0; } \\
& \text { zumb=1; }
\end{aligned}
$$

\}

else if $(z<=-0.0065 \& z>=-0.00862)$

\{

$$
\text { numb=1; }
$$

zumb=1;

\}

else if $(z<=-0.01088 \& \mathrm{z}>=-0.013)$

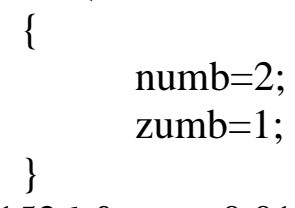

z)/humpdia $)^{*}$ pi $)+((\text { numb+1)*pi }))^{*}$ zumb;

zmod $=\sin (((((-$ numb $*$ humpgap $)-$

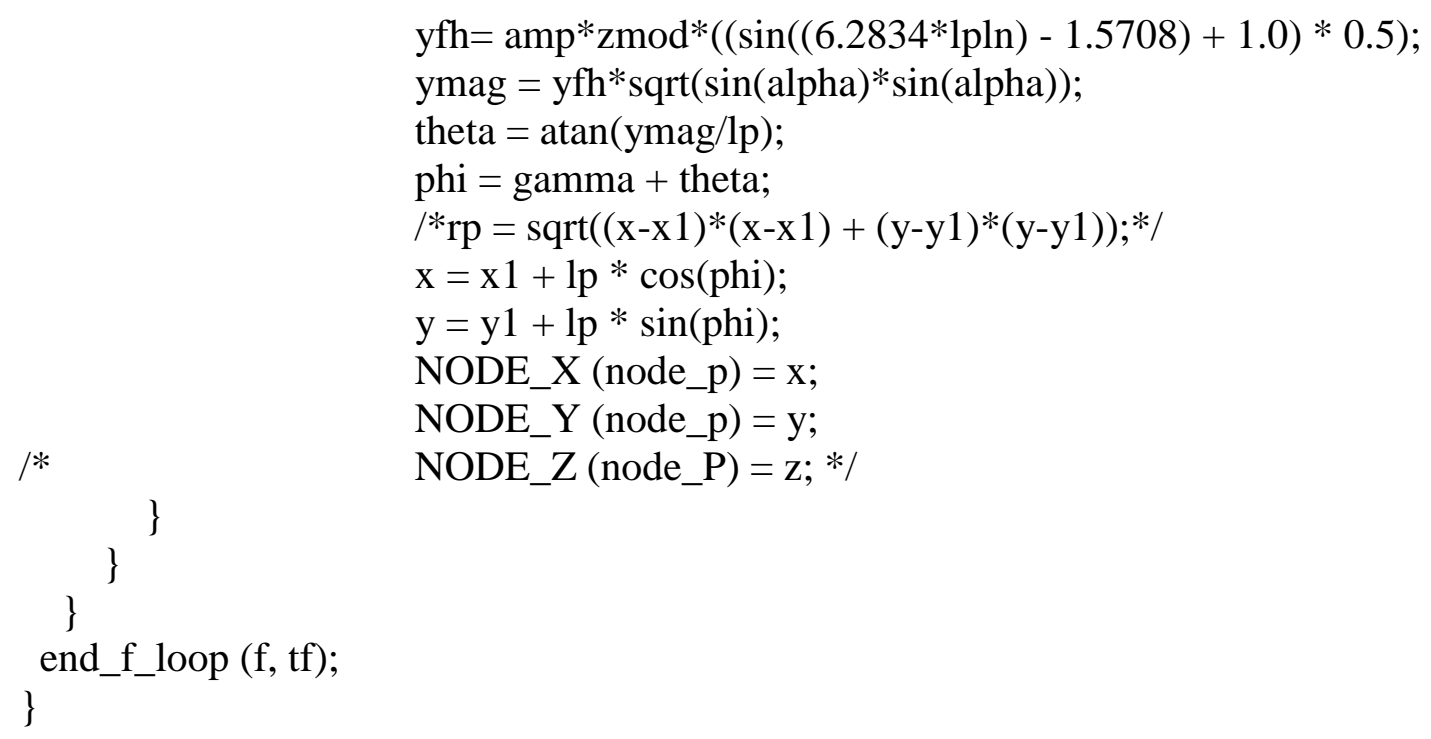


| $* * * * * * * * * * * * * * * * * * * * * * * * * * * * * * * * * * * * * * * * * * * * * * * * * * * * * * * * * * * * * * * * * * * * * 1$

/*

$/ * \quad$ End of the UDF.

/*

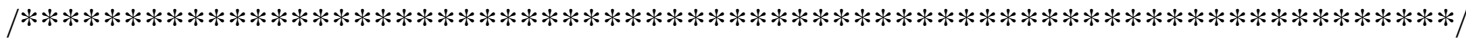

$* /$

*/ 